\title{
Low Emittance Electron Beam Studies
}

\author{
by \\ Rodion Tikhoplav \\ Submitted in Partial Fulfillment \\ of the \\ Requirements for the Degree \\ Doctor of Philosophy \\ Supervised by \\ Professor Adrian C. Melissinos \\ and \\ Professor Philippe Piot \\ Department of Physics and Astronomy \\ The College \\ Arts and Sciences \\ University of Rochester \\ Rochester, New York
}

2006 
Mariya,

my beloved bride and friend. 


\section{Curriculum Vitae}

The author was born in Omsk, Russia on August 3, 1973. He attended Moscow Engineering Physics Institute and graduated with a Diploma (BS) in 1996. He got his MS degree at Brigham Young University in 1999. He came to the University of Rochester in the Fall of 1999 and began graduate studies in Physics. In 2001 he moved to Fermi National Accelerator Laboratory and joined the A0 Photoinjector research team. He pursued his research in Physics under the direction of Professor Adrian Melissinos and received the Master of Arts degree from the University of Rochester in 2001. He presented his work at the EPAC 2002, LINAC 2004 and PAC 2005 conferences. 


\section{Acknowledgments}

I would like to sincerely thank my advisor professor Adrian Melissinos for his guidance, support and encouragement in my research work. I would also like to express my gratitude to professor Philippe Piot for his mentorship and supervision at A0. My special thanks to Helen Edwards for being a model of leadership. I owe a debt of gratitude to Todd Ballock and Jianliang Li for their laser contributions. I would like to especially thank Daniel Mehalcea and Grigory Kazakevich for their help in conducting experiments and data analysis. 


\section{Abstract}

We have studied the properties of a low emittance electron beam produced by laser pulses incident onto an rf gun photocathode. The experiments were carried out at the A0 photoinjector at Fermilab. Such beam studies are necessary for fixing the design of new Linear Colliders as well as for the development of Free Electron Lasers. An overview of the A0 photoinjector is given in Chapter 1. In Chapter 2 we describe the A0 photoinjector laser system. A stable laser system is imperative for reliable photoinjector operation. After the recent upgrade, we have been able to reach a new level of stability in the pulse-to-pulse fluctuations of the pulse amplitude, and of the temporal and transverse profiles. In Chapter 3 we present a study of transverse emittance versus the shape of the photo-cathode drive-laser pulse. For that purpose a special temporal profile laser shaping device called a pulse-stacker was developed. In Chapter 4 we discuss longitudinal beam dynamics studies using a two macro-particle bunch; this technique is helpful in analyzing pulse compression in the magnetic chicane, as well as velocity bunching effects in the rf-gun and the 9-cell accelerating cavity. In Chapter 5 we introduce a proposal for laser acceleration of electrons. We have developed a laser functioning

on the $\mathrm{TEM}_{01}^{*}$ mode, a mode with a longitudinal electric field component which is suitable for such a process. Using this technique at energies above $40 \mathrm{MeV}$, one would be able to observe laser-based acceleration. 


\section{Table of Contents}

List of Tables $\quad x$

List of Figures $\quad$ xi

1 The A0 Photoinjector 1

1.1 Introduction . . . . . . . . . . . . . . . . . . . 1

1.1.1 Linear Colliders . . . . . . . . . . . . . 2

1.1.2 Free Electron Lasers . . . . . . . . . . . . . . . . . . . . 2

1.1.3 Photoinjectors ................. . . 3

1.2 The Fermilab A0 Photoinjector . . . . . . . . . . . . 5

$1.3 \quad$ ILC . . . . . . . . . . . . . . . . . . . . . . 7

1.3.1 TESLA based ILC . . . . . . . . . . . . . . . . . 9

1.3 .2 TESLA FEL . . . . . . . . . . . . . . . . 10

1.4 Overview of the Activities at A0 . . . . . . . . . . . . 11

1.5 Thesis Outline ...................... 12

2 A0 Laser System $\quad 17$

2.1 Introduction . . . . . . . . . . . . . . . . 17 
2.2 Seed Laser . . . . . . . . . . . . . . . . . . . . . . . . . . . . . . . 23

2.3 Amplification and Conversion to UV . . . . . . . . . . . . 28

2.4 System Performance . . . . . . . . . . . . . . . . 32

2.4.1 Shot to Shot Fluctuations . . . . . . . . . . . . 32

2.4.2 Transverse and longitudinal profiles .......... 35

2.4.3 Long pulse train . . . . . . . . . . . . . 36

2.4.4 Transport to the cathode . . . . . . . . . . . 39

3 Manipulation of the Longitudinal Profile 41

3.1 Pulse Stacker . . . . . . . . . . . . . . . . . . . . . . . 43

3.2 Emittance . . . . . . . . . . . . . . . . . . 47

3.2.1 Introduction . . . . . . . . . . . . . 47

3.2.2 Sources of Emittance Growth . . . . . . . . . . . . 48

3.2.3 Experimental Set-up . . . . . . . . . . . . 54

3.2.4 Results .................... 57

3.3 Pulse length as a function of charge . . . . . . . . . . . . 60

3.4 Conclusion ...................... 60

4 Beam Compression $\quad 63$

4.1 Introduction . . . . . . . . . . . . . . . . 63

4.2 Theoretical Background ................ 64

4.2.1 Magnetic Bunch Compression ... . . . . . . . . 64

4.2.2 Velocity Bunching in the RF-Gun and 9-cell Cavity . . . . 70

4.2.3 Estimate of Space Charge Effects on the Separation between Two Macroparticles . . . . . . . . . . . . 70 
4.2 .4 Numerical Simulations . . . . . . . . . . . . . . . . 75

4.3 Experimental Set-up . . . . . . . . . . . . . . . 77

4.3.1 Magnetic Bunch Compression Measurement . . . . . . . 80

4.3.2 Velocity Bunching in RF-Gun and 9-cell Cavity . . . . . 84

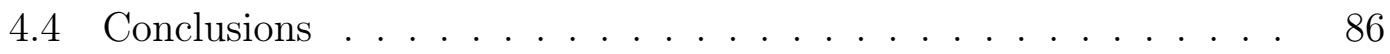

5 Laser Acceleration of Electrons $\quad 88$

5.1 Introduction . . . . . . . . . . . . . . . 88

5.2 The Open Iris-loaded Waveguide Structure and Phase Matching . 90

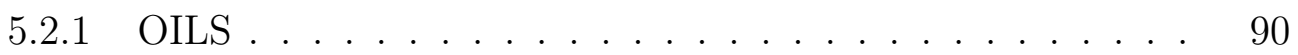

5.2 .2 Phase Matching . . . . . . . . . . . . . . . . . . . . . 92

5.3 Experimental Set-up . . . . . . . . . . . . . . . . . . . 94

5.3 .1 Laser System . . . . . . . . . . . . . . . . . . . . . . . 94

5.3 .2 Mach-Zender Interferometer . . . . . . . . . . . . . 98

5.4 Numerical Simulations . . . . . . . . . . . . . . . . . . . 102

5.5 Conclusion . . . . . . . . . . . . . . . . . . 105

$\begin{array}{ll}\text { Bibliography } & 106\end{array}$

$\begin{array}{ll}\text { A Vibration Measurements } & 110\end{array}$

A.1 Introduction . . . . . . . . . . . . . . 110

A.2 Description of the Measurements _. . . . . . . . . . 110

A.3 Analysis of the Results . . . . . . . . . . . . . . 115 
B Analysis of Frequency Doubling Efficiency 116

B.1 Introduction . . . . . . . . . . . . . . . 116

B.2 Theoretical Background . . . . . . . . . . . . . . . 117

B.3 Numerical simulation . . . . . . . . . . . . . . . . . . . . 117

B.4 Results . . . . . . . . . . . . . . . . . . . . 119

B.5 Conclusion . . . . . . . . . . . . . . . . . . . . . 119 


\section{List of Tables}

1.1 The operating settings of the A0 photoinjector. . . . . . . . . 5

1.2 TESLA X-ray FEL settings . . . . . . . . . . . . . 10

2.1 Laser parameters ................... . . 21

3.1 Linac operating parameters. . . . . . . . . . . . 56

4.1 Measured versus simulated $R_{56}$. The chicane current was $1.8 \mathrm{~A}$ (for the outer dipoles). . . . . . . . . . . . . . . . . . . . 84

5.1 Structure Parameters . . . . . . . . . . . . . . . . . . 90

5.2 A0 nominal operating settings and simulated parameters for $100 \mathrm{pC}$ bunch. ..................... 102

A.1 Vertical vibration, sensor $\# 1434$. . . . . . . . . . . . . . . . . . 111

A.2 Horizontal vibration, sensor \# 1429 . . . . . . . . . . . . . . . . . 113

A.3 Oscillator Table (One) when floated . . . . . . . . . . . . 113

B.1 Harmonic generation parameters. . . . . . . . . . . 118 


\section{List of Figures}

1.1 View of the A0 photoinjector inside the shielding cave. . . . . . . 6

1.2 Mechanical drawing of the A0 layout. . . . . . . . . . 8

1.3 Overview of the FNPL facility (red and magenta ellipses are respectively normal and skew quadrupole magnets; blue and orange rectangles represent dipole magnets). . . . . . . . . . . . 15

1.4 Schematic of the proposed ILC based on the TESLA design. . . . 16

2.1 Layout of the laser system, amplifier table (top) and crystal table (bottom). PP designates a Pulse Picker; FR, Faraday Rotator; 2P, two-pass amplifier; SF, spatial filter. CWAC designates continuous wave (as contrasted to single shot) autocorrelator and OSA is an optical spectrum analyzer. . . . . . . . . . . . . 18

2.2 A photograph of a new seed laser purchased from Time-Bandwidth, model GE-100 (Nd:YLF, 0.5 W, 5 ps, $\lambda=1054 \mathrm{~nm}) . \quad$. . . . . . 20

2.3 Transverse beam profiles before (left) and after (right) compensation by an anamorphic prism. The prism (not shown in Fig. 2.1) was inserted between the PP and the FR. . . . . . . . . . . 20 
2.4 Autocorrelation as measured with CWAC (top) and spectrum of the seed laser as measured with Optical Spectrum Analyzer (bottom). The pulse length FWHM was determined to be 7.23 ps, which implies an IR pulse length of 5.12 ps. The bandwidth FWHM was

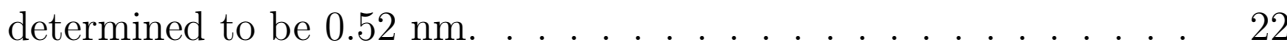

2.5 Block-diagram of the laser timing system. . . . . . . . . . 25

2.6 Schematic top view of multi-pass cavity. M1 and M2 are the flat and the curved mirrors respectively. The Q-switch and Brewster plate act as an input/output device. . . . . . . . . . .

2.7 Schematic top view of the two 2-pass amplifiers. A half-wave plate between the rods is used to balance the birefringence of the rods.

2.8 Single pulse amplitude vs. the number of round trips in the multipass cavity (top). Shot to shot fluctuations after the multi-pass amplifier measured for a different number of round trips (middle). Shot to shot fluctuations after the multi-pass amplifier and after frequency conversion to UV for the entire month of July 2005 (bottom). . . . . . . . . . . . . . . . . . . . .

2.9 Efficiency of the doubling (top) and quadrupling (bottom) crystals for different input pulse intensity. . . . . . . . . . . . . . .

2.10 UV beam profile measured on the target in the laser room and its projections. A $50 \mu \mathrm{m}$ spatial filter was inserted to improve the UV transverse profile. The beam size is about $4.3 \mathrm{~mm}$ FWHM. . . . 34

2.11 Picture of a single UV pulse recorded by the streak camera (left). Projection of the image on the vertical axis (right), and Gaussian fit to the data points. . . . . . . . . . . . . 
2.12 A long pulse train of 400 seed (IR) pulses captured with a fast digital oscilloscope (top); the same long pulse train after the 2-pass amplifiers (middle). And (bottom) a pulse train of $100 \mathrm{UV}$ pulses captured with a fast digital oscilloscope (green); the corresponding electron bunch train (blue). . . . . . . . . . . . . . . 37

2.13 Schematic diagram of the UV beam transport line. . . . . . . . 38

2.14 Five beamlets picture observed on the photocathode after the UV imaging relay system. . . . . . . . . . . . . . .

2.15 Dependence of the transverse electron bunch image on the solenoid current. In each pair of rows, the upper is experimental and the lower is simulation. The currents on the primary solenoid are, from left to right, 237 A, 209 A, 199 A (top pair of rows), 188 A, 182 A, and $173 \mathrm{~A}$ (bottom pair); the images are not to scale. . . . . .

3.1 The pulse-stacker principle of operation. A pulse-stacker takes a single Gaussian (left); splits it into four beams, and stacks the beams after introducing a delay between them (right). The four stacked Gaussians form a quasi flat-top distribution (blue). . . . .

3.2 Pulse Stacker; M-mirror, $\lambda / 2$-halfwave plate, $\mathrm{Pz}$-prism, SP-alternating polarizations. .................... . . . . 45

3.3 Time profile of the UV laser pulse measured by a streak camera. Five Gaussian (top) and eight flat-topped (bottom) laser shots and their average (thick blue). . . . . . . . . . . . . . . 46

3.4 Slit method schematics. The electrons coming from the left go through the slit (X3) and hit onto the YAG flag (X5). . . . . 
3.5 Beam line schematics (excerpt from Fig. 1.2). Emittance was measured at X3, which is the first available cross after the 9-cell cavity (left). The image of the slits at X3 was projected onto a YAG screen at X5. The numbers indicate the distance from the photocathode $($ in $\mathrm{mm}) \ldots \ldots \ldots \ldots \ldots \ldots$

3.6 Emittance versus solenoid field: experimental points (diamonds and circles) and ASTRA simulation (solid lines). Gaussian pulse length is $3.5 \mathrm{ps}$ rms and flat-top is $6.5 \mathrm{ps} \mathrm{rms}$; the transverse size for both is $0.9 \mathrm{~mm}$ rms and the charge is $1 \mathrm{nC} \ldots \ldots \ldots$

3.7 Image of slits at flag X5 (top) and its projection on the vertical axis (middle). Phase space correlation: divergence as a function of transverse distance (bottom). . . . . . . . . . . . .

3.8 Transverse phase space map at X3 (1 nC, 20.8 ps FWHM flattop, $\left.B_{\text {sol }}=0.137 \mathrm{~T}\right)$. Electron density distribution as a function of phase-space coordinates $\left(x, x^{\prime}\right)$. This plot corresponds to the projected emittance value of $4 \mathrm{~mm}$-mrad. To make the map readable, the slope of the phase space correlation (divergence of the whole electron beam, whose value is $0.4 \mathrm{mrad}$ ) was not included. . . .

3.9 Electron bunch length normalized to the corresponding laser pulse length (2.4 ps rms for Gaussian and $6.6 \mathrm{ps}$ rms for the flattop) as a function of charge. The growth for the longer flattop pulse is significantly slower. . . . . . . . . . . . . . .

4.1 Overview of the A0 magnetic bunch compressor chicane. The plane of the drawing corresponds to the deflecting plane $y z$. The magnetic fields $\left(B_{x}\right)$ are directed into the plane of paper. . . . . . . . 
4.2 Simplified block diagram of the A0 beamline. . . . . . . . . . .

4.3 Example of integration of equation of motion: vertical trajectory offset $y$ (top) and vertical deflection (middle) versus longitudinal coordinate. Bottom 2D field false color map of the chicane $B_{x}(y, s)$ field. . . . . . . . . . . . . . . . . . .

4.4 Streak camera profile of the laser set-up so as to create the twomacroparticle configuration. . . . . . . . . . . . .

4.5 Energy of the electron bunch downstream of the 9-cell cavity as a function of the 9-cell phase. The CTR signal rises above the background only at the maximum compression phase, $\Delta \phi=35^{\circ}$. .

4.6 Measurement of the energy separation between the two macroparticles. . . . . . . . . . . . . . . .

4.7 Scan of the phase between the rf-gun and the photocathode drivelaser when only leading (trailing) or both pulses are incident on the

4.8 Calculated $R_{56}$ for three experimentally measured cases: $12.9 \mathrm{MeV}$ (top), 14.3 MeV (middle) and $14.5 \mathrm{MeV}$ (bottom). . . . . . . .

4.9 Velocity bunching in the rf-gun and 9-cell cavity. Compression ratio as a function of rf-gun phase (top) and 9-cell phase (bottom). Diamonds are experimental measurements and the dashed line corresponds to numerical simulations. . . . . . . . . . . .

5.1 OILS Waveguide: radius $a=0.5 \mathrm{~mm}$, element length $L=2 \mathrm{~mm}$, angle of tapering $\alpha=20^{\circ} \ldots \ldots \ldots \ldots \ldots \ldots$

5.2 Sensitivity of energy gain on gas pressure (a) and required gas pres-

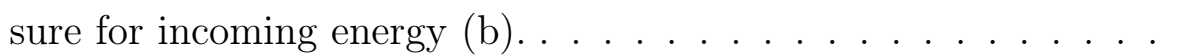


5.3 Schematics of the oscillator (top) and re-gen amplifier (bottom). M1, high reflectivity curved mirror; M2, output coupler (80\% transmission mirror); BP, Brewster polarizer; A, aperture; ML, mode locker; Q-sw, Q-switch Pockel cell; W, wire (to suppress the fundamental mode). . . . . . . . . . . . . . . . .

5.4 Doughnut shape mode $\left(T E M_{01}^{*}\right)$ is achieved as a linear combination of $\left(T E M_{01}\right)$ and $\left(T E M_{10}\right)$ modes with proper phase-matching. .

5.5 Mach-Zender Interferometer: 50/50 beam splitter $B S$, periscopes $P S 1$ and $P S 2$, combining cube $C C$, polarizer $P$, half-wave plate $P l$, piezo-driven mirror $M . \ldots \ldots \ldots \ldots$

5.6 Measured transverse density a) and corresponding line profile b) of the $\mathrm{TEM}_{01}^{*}$ mode. . . . . . . . . . . . . . . . . . . . . . . . . 99

5.7 The phase matching test: polarizer oriented at different angles $(0$, 45, 90 and 135 degrees). The polarizer makes the doughnut-shape mode $\left(T E M_{01}^{*}\right)$ collapse back to the $T E M_{01}$ mode losing its cylindrical symmetry; its orientation should correspond to the polarizer orientation for all angles. . . . . . . . . . . . . . . .

5.8 The laser beam enters from the top left. After propagating passing through a lens (L1) and reflecting from the apertured mirror (M1), the beam co-propagates with the electron beam through the open iris-loaded structure (OILS) [green rectangle]. The laser beam is then extracted from the chamber thanks to a second apertured mirror (M2) and then transported to a diagnostics station. . . . . 101 
5.9 Simulated energy spectrum for various electron beam sizes in the OILS structure (left) and corresponding energy spread (right). Note for $\sigma_{x, y}>0.2 \mathrm{~mm}$, the beam is not fully transmitted $\left(\sigma_{E}=10 \mathrm{keV}\right)$. (Note that the profile corresponding to the "laser off" case has been scaled by $1 / 10)$. . . . . . . . . . . . . . . . . . . . 101

5.10 Energy and density modulation: (top) longitudinal phase space upstream (input), and at different locations downstream of the OILS section entrance $(5,10$ and $20 \mathrm{~cm})$ along with the associated charge density (bottom) $(s>0$ corresponds to the bunch head). . . . . . 104

5.11 Sensitivity of the energy spectrum on the incoming electron beam energy spread $\left(\sigma_{x, y}=0.1 \mathrm{~mm}\right) . \ldots \ldots \ldots 10 . \ldots \ldots$

A.1 A typical Lecroy oscilloscope trace of a velocimeter in the laser room. The upper trace corresponds to the signal, the lower to its

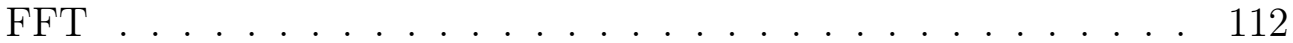

A.2 Integrated vibration measurements in the A0 (solid line) and APS (broken line) facilities. Integration goes from high to low frequency. "Piezo" and "geo" stand for the accelerometer and velocimeter measurements respectively. Floor was measured by velocimeter only. .

B.1 Doubling (top) and quadrupling (bottom) efficiency simulations and experimental data. The non-uniformity of the wave front is modeled by the tilt angle. . . . . . . . . . . . . . . 120 


\section{List of Abbreviations}

\begin{tabular}{ll} 
BBO & beta-barium borate \\
CERN & European Council for Nuclear Research \\
CCD & charge-coupled device \\
CPA & chirp pulse amplification \\
CTR & coherent transition radiation \\
CWAC & continuous wave autocorrelator \\
DC & direct current \\
DESY & Deutsches Elektronen Synchrotron \\
EPAC & European Particle Accelerator Conference \\
FEL & free electron laser \\
FWHM & full width at half maximum \\
GPIB & General Purpose Instrumentation Bus \\
GVD & group velocity dispersion \\
ILC & International Linear Collider \\
IR & infrared \\
KDP & potassium dihydrogen phosphate \\
LEP & Large Electron-Positron Collider \\
LBNL & Lawrence Berkeley National Laboratory \\
LHC & Large Hadron Collider \\
linac & linear accelerator \\
\hline
\end{tabular}




$\begin{array}{ll}\text { LINAC } & \text { Linear Accelerator Conference } \\ \text { OILS } & \text { open iris-loaded structure } \\ \text { OSA } & \text { optical spectrum analyzer } \\ \text { OTR } & \text { optical transition radiation } \\ \text { PAC } & \text { Particle Accelerator Conference } \\ \text { QCD } & \text { quantum chromodynamics } \\ \text { regen } & \text { regenerative amplifier } \\ \text { rf } & \text { radio frequency } \\ \text { rms } & \text { root mean square } \\ \text { SASE } & \text { self-amplified stimulated-emission } \\ \text { SLAC } & \text { Stanford Linear Accelerator Center } \\ \text { TEM } & \text { transverse electromagnetic wave } \\ \text { TESLA } & \text { TeV-Energy Superconducting Linear Accelerator } \\ \text { UV } & \text { ultraviolet } \\ \text { YAG } & \text { yttrium aluminum garnet } \\ \text { YLF } & \text { yttrium lithium fluoride }\end{array}$




\section{Chapter 1}

\section{The A0 Photoinjector}

\section{$1.1 \quad$ Introduction}

Humans are never satisfied with what has been accomplished. There is always a desire to extend the horizons of knowledge even farther. To satisfy the scientific curiosity, as well as to push our civilization forward by investigating new physics phenomena, we need to continue improving the physics devices at our disposal, including high energy particle accelerators.

Since the introduction of the Van de Graaff generator [1] in 1929 and of the Betatron [2] in 1940, accelerators became standard tools for physics application ranging from probing QCD phenomena within the realm of High Energy Physics to biophysics studies by means of free electron lasers. And even now, seventy years later, high energy particle accelerators still remain the best tools to address relevant questions in modern physics from the identity of dark matter to the existence of extra dimensions. 


\subsubsection{Linear Colliders}

Colliders are particle accelerator-type machines [3] which allow physicists to study fundamental laws of nature by colliding fast moving particles with each other (such as electrons against positrons, or protons against protons or antiprotons). As a result of such collisions new particles are created, provided that their rest mass is not greater than the center of mass energy of the incoming particles. By studying newly created particles, as well as their decay products, one can recover the processes which guide the sub-atomic world and thus better understand the Universe.

There are two basic configurations of accelerators: linear accelerators (Linacs) and circular machines (Synchrotrons). The latter allows particles to gain greater energy after undergoing many revolutions in the accelerator. Circular machines are well suited for particles with great mass over charge ratio such as protons. For electrons, this ratio is three orders of magnitude smaller and moving in a circular orbit results in a great energy loss due to the synchrotron radiation. The last circular electron storage ring, LEP at CERN had circumference of $28 \mathrm{~km}$ and reached electron-positron center of mass energy of $120 \mathrm{GeV}$. Thus, for very high energies, electron-positron linacs are the only possible venue.

\subsubsection{Free Electron Lasers}

Introduced more than thirty years ago [4][5], a free electron laser or FEL, generates tunable, coherent, high power radiation, currently ranging in wavelength from millimeters to the UV and even X-rays. While an FEL beam shares the same optical properties as conventional lasers, namely the radiation is monochromatic and coherent, the operation of an FEL is quite different. Unlike gas or diode lasers 
which rely on bound atomic or molecular states, FELs use a relativistic electron beam as the lasing medium, hence the term "free-electron".

To produce radiation in an FEL, a relativistic beam of electrons passes through a periodic, transverse magnetic field. This field is produced by a set of magnets with alternating poles along the beam path, called a "wiggler" because it forces the electrons in the beam to propagate along a sinusoidal path. The acceleration of the electrons along this path results in the emission of photons. Viewed relativistically in the rest frame of the electron, the magnetic field can be treated as an ensemble of virtual photons. The collision of an electron with a virtual photon creates a real photon due to Compton scattering. Mirrors capture the released photons whose motion is in phase with the field of the light already emitted, so that the fields add coherently thus truly making it a laser. The wavelength $\lambda$ of FEL radiation is related to the period length $\lambda_{u}$ of an undulator by

$$
\lambda=\frac{\lambda_{u}}{2 \gamma^{2}}\left(1+\frac{K^{2}}{2}\right) .
$$

where $\gamma=E / m c^{2}$ is the relativistic factor of the electrons, $K=e B_{u} \lambda_{u} /(2 \pi m c)$ is the undulator parameter, and $B_{u}$ is the peak magnetic field in the undulator [6]. Adjusting either the beam energy or the field strength tunes the wavelength over a wide range.

\subsubsection{Photoinjectors}

A photoinjector is, often, the first stage in modern electron linear accelerator. It consists of a "photo-gun" housing a laser-driven photocathode to produce and immediately accelerate electron bunches to a few $\mathrm{MeV}$ so as to preserve low emittance. There are two types of photo-guns: DC, which use a constant electric field, as in a capacitor, and radio frequency ( $\mathrm{rf})$, which use short $(1 / 2-2$ cells) waveguide 
cavities as an accelerating structure. The latter can provide a more than an order of magnitude higher accelerating gradient $(35 \mathrm{MV} / \mathrm{m})$ and is therefore preferable, since faster acceleration results in an electron beam with better transverse and longitudinal characteristics. It is not necessary to operate the rf gun and multi-cell cavities at the same frequency, however it is more convenient.

Linac concepts considered by the linear collider community today, mainly differ with respect to the choice of rf frequency. Simply put, higher rf frequencies (such as $11.4 \mathrm{GHz}$ X-band, developed by the SLAC collaboration [7][8]) offer a higher accelerating gradient, thus a shorter beam-line length (and lower cost of construction). Lower rf frequencies (such as $1.3 \mathrm{GHz}$ L-band) promise a very good beam quality, and if operated in the superconductive regime (as developed by the TESLA collaboration [9]), much lower power consumption (lower cost of operation). The L-band superconductive technology can also sustain longer pulse trains thus providing higher luminosity. The luminosity is defined such that a process of cross section $\sigma$ will occur $\mathcal{L} \sigma$ times per second, where

$$
\mathcal{L}=\frac{N_{+} N_{-}}{4 \pi \sigma_{x} \sigma_{y}} f
$$

Here $f$ is the collision frequency equal to the product of machine frequency and number of bunches, and $N_{+,-}$is the number of particles per bunch; $\sigma_{x, y}$ is the transverse rms size of a bunch [10] [11]. After careful analysis by the International Technology Recommendation Panel, L-band superconductive technology was chosen for the next linear collider.

The A0 superconducting L-band rf photoinjector (Fig. 1.1) is a project at Fermilab built in collaboration with the TESLA test facility in DESY Hamburg, designed to prototype the low-energy stage of the next-generation International Linear Collider (ILC). 


\subsection{The Fermilab A0 Photoinjector}

The A0 photoinjector [12] consists of a photoemission electron source based on an $1+1 / 2$ cell $\mathrm{L}$ band $(1.3 \mathrm{GHz})$ rf-gun. The CsTe photocathode is illuminated by an ultrashort (5 ps) UV laser pulse. The electron energy after the gun is $\sim 4 \mathrm{MeV}$. This is followed by a 9-cell superconducting Niobium cavity cooled by superfluid Helium to $T=2 \mathrm{~K}$ and operating with a nominal accelerating gradient of $12 \mathrm{MV} / \mathrm{m}$, and beam focusing elements and diagnostics to handle the $16 \mathrm{MeV}$ low emittance electron beam. The mechanical drawing of $\mathrm{A} 0$ and a schematic drawing of the beam line are shown in Figs. 1.2 and 1.3 respectively. The advantage of using superconducting cavities is immediately manifested in the

Table 1.1: The operating settings of the A0 photoinjector.

\begin{tabular}{|c|c|c|}
\hline Parameter & Value & Unit \\
\hline peak gun field & 35 & $\mathrm{MV} / \mathrm{m}$ \\
\hline laser launch phase & 40 wrt zero-cros. & $\mathrm{deg}$ \\
\hline 9-cell cavity accel. field & 12.5 & $\mathrm{MV} / \mathrm{m}$ \\
\hline 9-cell cavity phase & 0 (on-crest ) & $\mathrm{deg}$ \\
\hline rms laser pulse length & 2 & $\mathrm{ps}$ \\
\hline rms laser spot size & 1 & $\mathrm{~mm}$ \\
\hline charge & $1-20$ & $\mathrm{nC}$ \\
\hline total energy & 16 & $\mathrm{MeV}$ \\
\hline bunch length & $0.5(1.7)$ & $\mathrm{mm}(\mathrm{ps})$ \\
\hline momentum spread $(1 \mathrm{nC})$ & 35 & $\mathrm{keV} / \mathrm{c}$ \\
\hline
\end{tabular}

power consumption. The power required to operate the 9-cell cavity (no more than $200 \mathrm{~kW}$ ) is only one tenth of that required for the normal conducting rf-gun. 


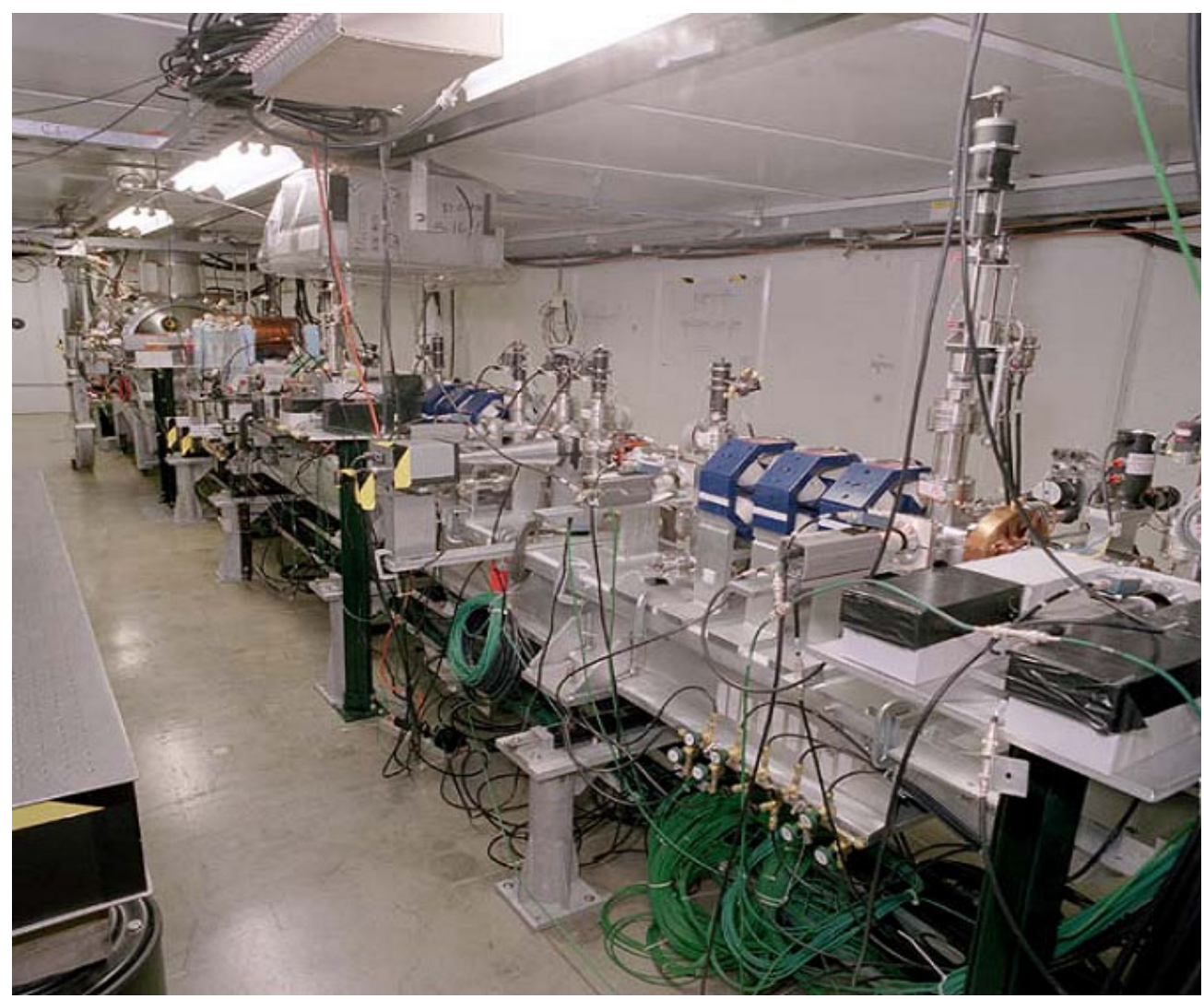

Figure 1.1: View of the A0 photoinjector inside the shielding cave. 
Beam charge as high as $20 \mathrm{nC}$ per bunch can be delivered. The operating settings of the A0 photoinjector are given in Table 1.1.

Downstream of the cavity the beam line includes a set of quadrupoles and steering dipole elements for beam focusing and orbit correction, a skew quadrupole channel that allows the generation of flat beams using an incoming angularmomentum dominated beam, and a magnetic bunch compressor chicane which can enhance the bunch peak current up to $2.5 \mathrm{kA}$. The diagnostics for measuring transverse beam properties consist of electromagnetic beam position monitors, optical transition radiation or yttrium aluminum garnet (YAG) screens (for measuring beam transverse density) and three emittance measurements stations based on the multi-slit mask technique. The bunch length measurement is performed by a streak camera that streaks optical transition radiation pulses emitted by the bunch. The streak camera is a Hamamatsu C5680-21S equipped with a M5676 fast sweep module and a pulnix progressive scan digital CCD camera. The streak camera calibration, when operated at the highest sweep speed is $3.0 \mathrm{pixel} / \mathrm{ps}$. An alternative frequency-domain bunch length diagnostics based on Martin-Puplett interferometry of coherent transition radiation is also available. Downstream of the beamline, the beam can be bent in a dispersive section, to measure the beam energy distribution, or transported to the user experimental area. The A0 facility can be operated remotely. So far teams from LBNL and DESY have used this capability to remotely perform beam physics experiments.

\section{$1.3 \quad$ ILC}

Developed in the early 1970s, the standard model of particle physics is a theory which describes the fundamental particles and their interactions through strong, 


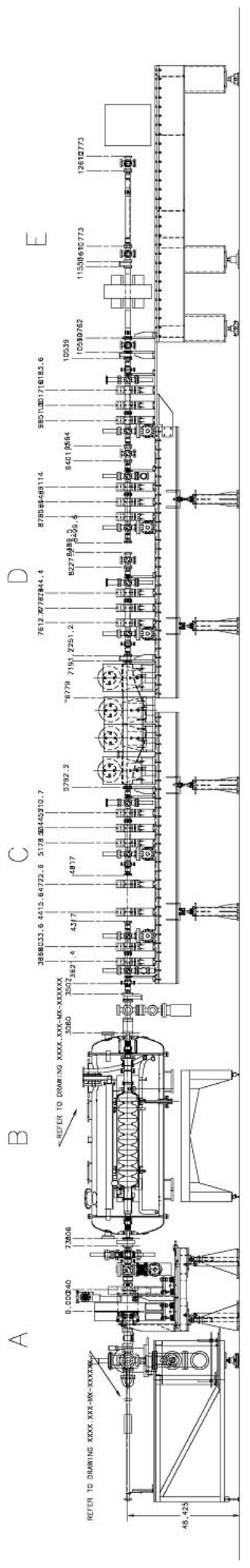

Figure 1.2: Mechanical drawing of the A0 layout. 
weak, and electromagnetic forces. Its predictions have been confirmed by a number of experiments. Nevertheless, there are phenomena that lay outside the realm of the standard model, such as dark matter, dark energy and neutrino mass, that need further exploration. The first step towards this exploration is finding the Higgs boson, which is a quantum of the Higgs field, the field that is responsible for the mass associated with each elementary particle. If it exists, the Higgs should be discovered at the Large Hadron Collider at CERN (a $28 \mathrm{~km}$ circular proton-proton collider with the center of mass energy of $14 \mathrm{TeV}$ ). However, in order to study the properties of the Higgs and other possibly discovered particles, a complementary tool, namely a 250 on $250 \mathrm{GeV}$ electron-positron linear collider is needed. This is because electrons and positrons interact only through the electroweak force, thus the experiments are much "cleaner". The ILC is a proposed new electronpositron collider. Together with the Large Hadron Collider at CERN, it would allow physicists to explore energy regions beyond the reach of today's accelerators.

\subsubsection{TESLA based ILC}

There are three possible countries where the ILC can be hosted: USA, Germany and Japan. If the USA is chosen to be the host, the most probable site is Fermilab; should Germany be chosen, the ILC will be based on the TESLA infrastructure.

A schematic view of the ILC based on the TESLA design is shown in Fig. 1.4. The electron beam is generated in a polarized laser-driven DC gun, and after being accelerated to the energy of $5 \mathrm{GeV}$ it enters the damping ring, whose purpose is to improve emittance due to synchrotron radiation as the electron beam passes through wigglers. After acquiring the needed transverse and longitudinal characteristics the beam enters the main linac and is accelerated to an energy of $250 \mathrm{GeV}$, where it collides with the positron beam at the same energy. The 
positrons, created at the positron source, go through analogous acceleration steps. The positrons are produced from $\gamma$ conversion in a thin ( 0.4 of a radiation length $X_{0}$ ) Ti-alloy target. The target is rotating to reduce the thermal stress from a long bunch train.

\subsubsection{TESLA FEL}

As was discussed in section 1.1.2, FELs produce photons over a wide range of wavelengths. From Eq. 1.1 it follows that $\lambda$ is inversely proportional to $\gamma^{2}$ thus, it is possible to achieve a very short wavelength by using a highly energetic electron beam.

Table 1.2: TESLA X-ray FEL settings

\begin{tabular}{|l|l|}
\hline Parameter & Value \\
\hline Electron energy & $10-20 \mathrm{GeV}$ \\
\hline Wavelength of X-ray radiation & 6 to $0.085 \mathrm{~nm}$ \\
\hline Peak power & $37 \mathrm{GW}$ \\
\hline Average power & $210 \mathrm{~W}$ \\
\hline Bandwidth (FWHM) & $0.08 \%$ \\
\hline Photon beam size (FWHM) & $0.1 \mathrm{~mm}$ \\
\hline Pulse duration (FWHM) & $100 \mathrm{fs}$ \\
\hline
\end{tabular}

The TESLA Test Facility has established a record by achieving self amplified spontaneous emission (SASE) at $100 \mathrm{~nm}$ [13]. The SASE FEL principle of operation is based on amplification in a single pass of the spontaneous radiation emitted in the beginning of the undulator. Thus it does not require any mirrors and can operate in the X-ray region. 
Due to its ability to preserve a high beam quality during acceleration, the TESLA linac is an excellent choice for an X-ray FEL. Table 1.2 gives typical settings of the TESLA X-ray FEL after the project is completed in 2012 [9]. At SLAC, the existing high energy electron linac will be used to drive an X-ray FEL [14].

The intense X-ray pulses would enable researchers to analyze the dynamics of processes on a sub-atomic scale, such as, the progress of a chemical reaction, the movement of biomolecules, and the formation of solids. This would benefit a wide range of scientists as well as industrial users.

\subsection{Overview of the Activities at A0}

The A0 photoinjector, with its high charge and low emittance $16 \mathrm{MeV}$ beam, is a unique facility for accelerator physics reseach. Several advanced beam dynamics experiments have been pursued at the A0 photoinjector.

Past experiments include:

Electro-optical sampling of electron beams [15]. This method can be used to measure the bunch length and time profile of picosecond electron bunches based on the fact that certain crystals (such as KDP, LiTa etc.) change their optical properties (birefringence) in the presence of an external electric field.

Photoinjector rf gun studies, including the gun dark current and the Cesium Telluride photocathode quantum efficiency as a function of time and the rf [16]. Improving the photocathode performance is essential for delivering high charge/high current beams.

Present activities at $\mathrm{A} 0$ include:

Photoinjector production of angular momentum dominated electron beams and 
subsequent generation of a flat beam with high transverse emittance ratio [17]. A flat beam is of interest for high-energy electron-positron colliders or acceleratorbased light sources. For bunch charge of $0.5 \mathrm{nC}$, an emittance ratio of 100 was measured [18].

Collaborators from NIU and UCLA have performed an experiment on plasmawake field acceleration. The experiment consists of injecting a high charge (typically $10 \mathrm{nC}$ ) short (typically $3 \mathrm{ps)}$ ) electron bunch in an Argon plasma. The experiment has demonstrated both beam deceleration and acceleration in plasma. From this experiment the amplitude of the accelerating plasma wake-field has been measured to be $130 \mathrm{MV} / \mathrm{m}$ [19]. Our UCLA collaborators have also installed an experiment to realize an electron source based on the plasma-density transition [20]. A first set of experiments was attempted and the experimental apparatus has been used to study the focusing properties of a plasma lens operating in the under-dense regime.

The Urbana-Champaign team has installed a fast kicker to demonstrate a prototype being considered for operating with short damping rings [22].

The beam dynamics studies conducted by the author include studies of transverse emittance as well as of longitudinal beam dynamics using a novel twomacroparticle technique, and extensive laser work, relevant both to the A0 operation as well as to advanced accelerator methods.

\subsection{Thesis Outline}

In Chapter 2, we describe the A0 photoinjector laser system. A stable laser system is imperative for reliable photoinjector operation. To improve the stability a new picosecond seed laser was installed; that allowed us to bypass a $2 \mathrm{~km}$ fiber 
stretcher and grating compressor, thus improving pulse-to-pulse fluctuations as well as the temporal and transverse profiles of the final beam. An imaging relay was introduced between the laser components thus preserving the flat wave front, and consequently improving frequency doubling and quadrupling efficiency resulting in higher charge output. To transport the UV beam onto the photocathode, an imaging system was built, which allows us to introduce controlled transverse patterns on the electron gun.

In Chapter 3, we present studies of transverse emittance and electron beam pulse length for various operating points of the electron source: electron beam charge, laser length and spot size, and solenoid settings. Emittance minimization is important for a number of applications especially for optimization of a free electron laser. We especially investigate the impact, on transverse emittance, of short Gaussian versus long square temporal distributions of the photocathode drive-laser. For that purpose, a special temporal profile laser shaping device called a "pulse-stacker" was built and installed.

In Chapter 4, we discuss longitudinal beam dynamics studies using a two macro-particle bunch. The distance between the two macro-particles is short enough to fall into the same rf bucket and thus they can be treated as a single bunch. At the same time the spacing is long enough to detect the change in distance between the particles using the state of the art streak camera and coherent transition radiation methods. The two macro-particle technique is helpful for the chicane compression studies as well as for velocity bunching studies in the rf-gun and the 9-cell cavity. Numerical simulation programs were used to confirm the experimental results of the studies.

In Chapter 5, we introduce a proposal for laser acceleration of electrons. Advanced acceleration methods have a great potential for the long term $R \& D$ of 
accelerator physics. We developed a laser system operating in the $\mathrm{TEM}_{01}^{*}$ mode, which has a longitudinal electric field component. After the foreseen energy upgrade of $\mathrm{A} 0$, this technique can be used to demonstrate laser-based acceleration at energies above $40 \mathrm{MeV}$.

The appendices are related to laser system performance studies. In Appendix A, we present quantitative measures of the vibration in the laser room in order to mitigate it in future installations. Appendix B describes a numerical simulation program for frequency doubling and quadrupling in crystals. 


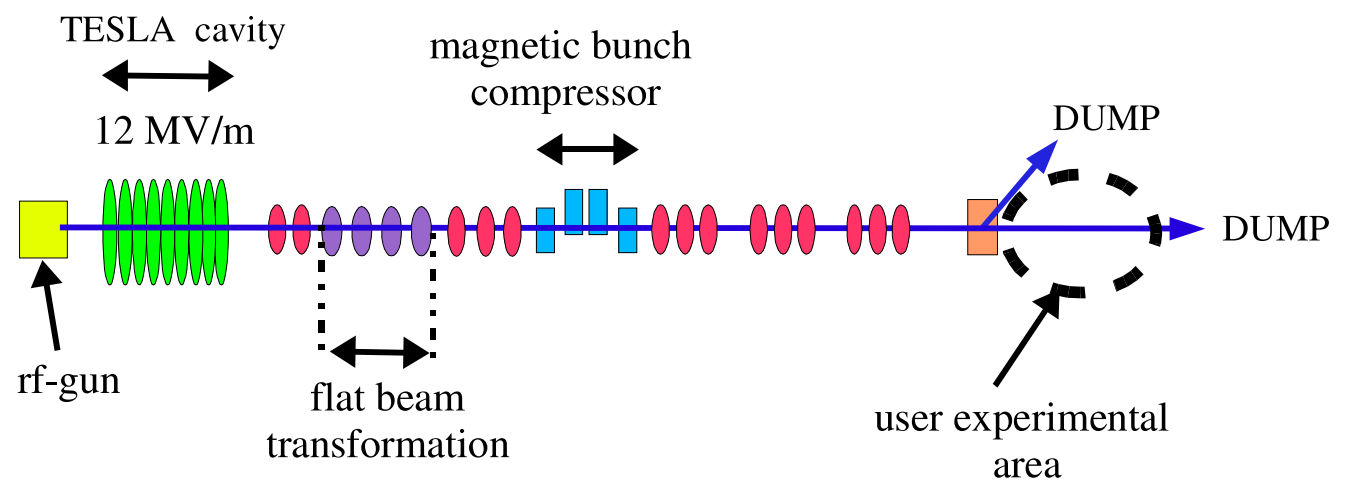

Figure 1.3: Overview of the FNPL facility (red and magenta ellipses are respectively normal and skew quadrupole magnets; blue and orange rectangles represent dipole magnets). 


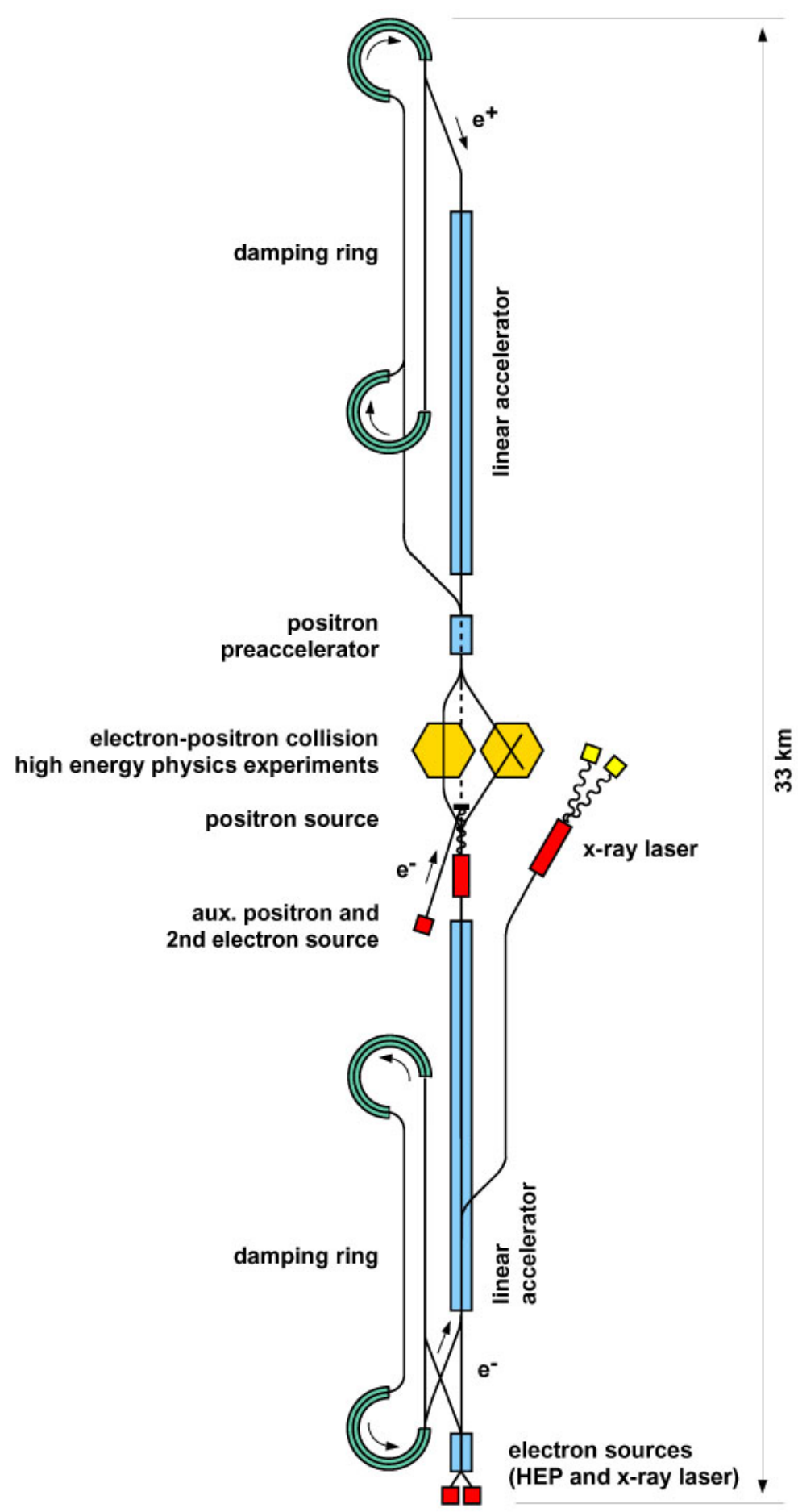

Figure 1.4: Schematic of the proposed ILC based on the TESLA design. 


\section{Chapter 2}

\section{A0 Laser System}

\section{$2.1 \quad$ Introduction}

The A0 photoinjector laser system was designed, assembled and supported by the University of Rochester laser team. The first version of the drive laser was installed in 1998 [21] and has been in operation since then, but was limited in certain aspects. Most seriously, the bandwidth of the seed pulse, which was generated in a $2 \mathrm{~km}$ long fiber, was unstable due to environmental fluctuations. The compression ratio of the grating compressor consisting of a pair of gratings, which were aligned for a fixed bandwidth, is dependent on the bandwidth of the input beam, and, hence, the output pulse duration was unstable. The instability of the pulse length resulted in serious amplitude fluctuations when the frequency of the IR beam was doubled and quadrupled into the UV. Furthermore, it was difficult to maintain optimal coupling of the seed pulse into the multi-pass amplifier, requiring the use of a large number of round trips (as many as 13) to achieve the desired gain. As discussed later this contributes significantly to pulse to pulse fluctuations. The absence of image relay in the optical system resulted in degradation of the 


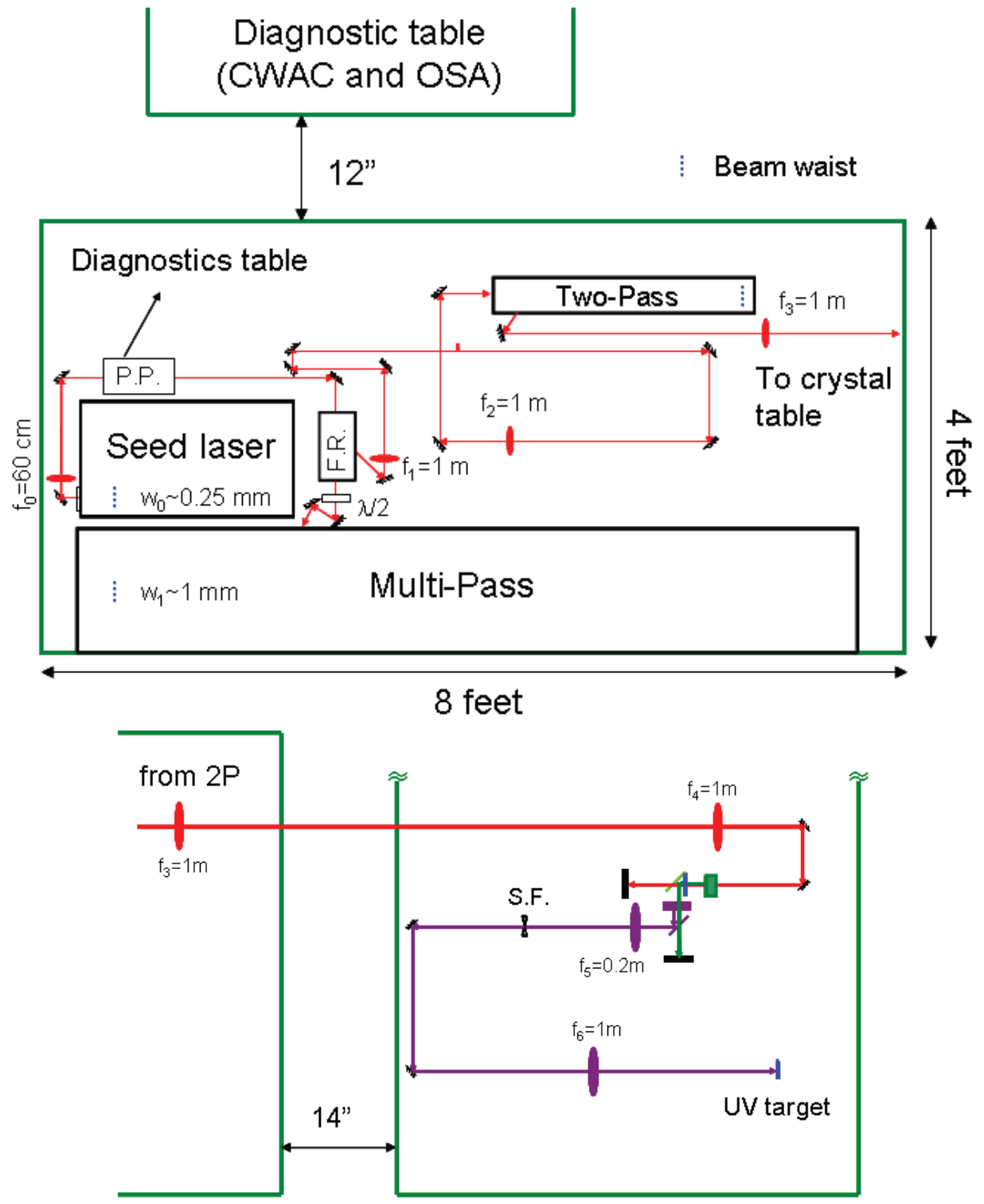

Figure 2.1: Layout of the laser system, amplifier table (top) and crystal table (bottom). PP designates a Pulse Picker; FR, Faraday Rotator; 2P, two-pass amplifier; SF, spatial filter. CWAC designates continuous wave (as contrasted to single shot) autocorrelator and OSA is an optical spectrum analyzer. 
wavefront, with corresponding decrease in the efficiency for frequency doubling and quadrupling. It also contributed to an inadequate transverse UV profile on the cathode. To resolve the above problems the laser system was upgraded in Spring of 2005.

The upgraded laser system is shown in Fig. 2.1, and is located on two $4 \times 8$ feet optical tables. The oscillator [24] $(\lambda=1054 \mathrm{~nm})$ delivering $450 \mathrm{~mW}$ of $5 \mathrm{ps}$ long pulses at $81.25 \mathrm{MHz}$, is phase locked to the master oscillator that controls the L-band rf. A single pulse is selected out of this train by a pulse picker [25] and amplified in a multi-pass laser cavity with a flash lamp pumped $15 \mathrm{~cm} \mathrm{Nd:glass} \mathrm{rod}$ as the gain medium. The output of the multi-pass amplifier is further amplified in two 2-pass glass amplifiers. The frequency of the IR pulse is then doubled and quadrupled in two $10 \mathrm{~mm} \mathrm{BBO}$ crystals to the $\mathrm{UV}(\lambda=263.5 \mathrm{~nm})$. Finally, the UV pulse is transported to the cathode at a distance of $20 \mathrm{~m}$ from the laser room. The laser parameters are summarized in Table 2.1. Presently, up to 800 pulses spaced at $1 \mu$ s can be delivered at a repetition rate at $1 \mathrm{~Hz}$. A similar system with higher repetition rate is operating at the DESY Test Facility [26] [27].

In the following sections we discuss the individual components as well as the overall performance of the laser system. We begin by describing the characterization of the new seed laser. In the third section we present details on the amplification of the seed pulse in the multi-pass and 2-pass amplifiers. The IR beam waist is relayed to the doubling and quadrupling crystals so that the wavefront remains flat, leading to much improved conversion efficiencies in the crystals. The quality of the UV beam was optimized by introducing a $50 \mu$ diameter spatial filter inserted at the focal point of the UV telescope.

The final section is divided into several subsections that cover different aspects of the system's performance. We first discuss pulse to pulse fluctuations. By 


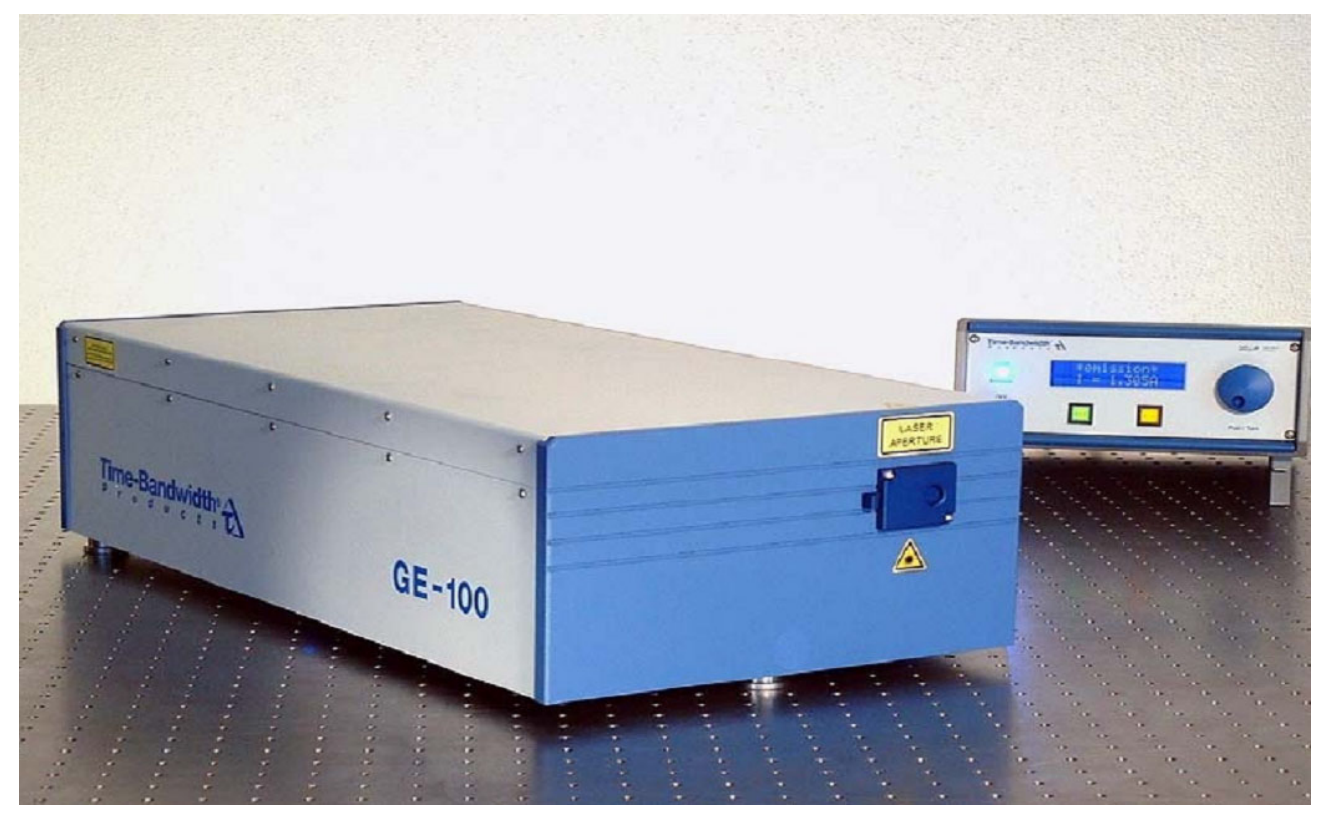

Figure 2.2: A photograph of a new seed laser purchased from Time-Bandwidth, model GE-100 (Nd:YLF, 0.5 W, 5 ps, $\lambda=1054 \mathrm{~nm})$.

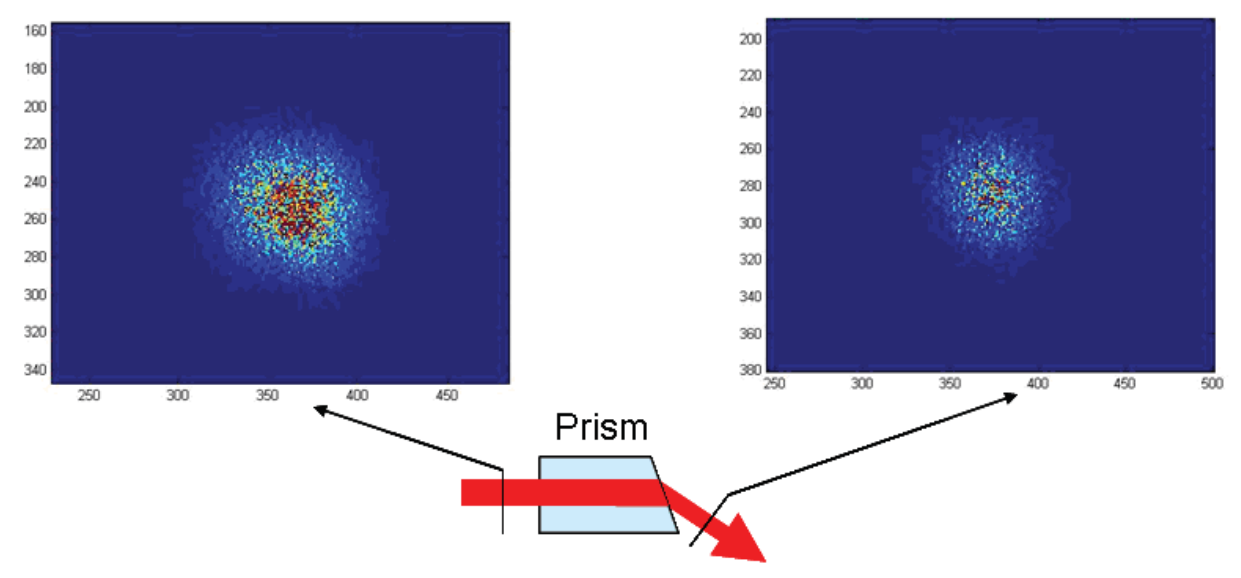

Figure 2.3: Transverse beam profiles before (left) and after (right) compensation by an anamorphic prism. The prism (not shown in Fig. 2.1) was inserted between the PP and the FR. 
Table 2.1: Laser parameters

\begin{tabular}{|l|l|}
\hline Oscillator frequency & $81.25 \mathrm{MHz}$ \\
\hline Oscillator wavelength & $1054 \mathrm{~nm}$ \\
\hline Oscillator energy/pulse & $5.5 \mathrm{~nJ}$ \\
\hline Energy/pulse after multi-pass & $6 \mu \mathrm{J}$ \\
\hline Energy/pulse after two-pass & $100 \mu \mathrm{J}$ \\
\hline UV energy/pulse after crystals & $20 \mu \mathrm{J}$ \\
\hline UV energy/pulse on cathode & $10 \mu \mathrm{J}$ \\
\hline UV pulse length (FWHM) & $5 \mathrm{ps}$ \\
\hline Separation of pulses in train & $1 \mu \mathrm{s}$ \\
\hline Length of pulse train & up to 800 pulses \\
\hline Repetition rate & $1 \mathrm{~Hz}$ \\
\hline
\end{tabular}

comparing the amplitude fluctuations after the multi-pass amplifier and in the UV, we conclude that the primary source of the fluctuations is the instability in the power supply driving the flash lamp of the multi-pass amplifier. The transverse and longitudinal profiles of the UV beam which were measured using a CCD camera and a streak camera are presented. We also show data from a long UV pulse train with 400 pulses. This pulse train can be extended to 800 pulses and can be made flat by preshaping the oscillator pulse train before injection into the multi-pass amplifier. To transport the UV beam onto the photocathode, an imaging system was built, which allows us to introduce controlled transverse patterns on the electron gun. 

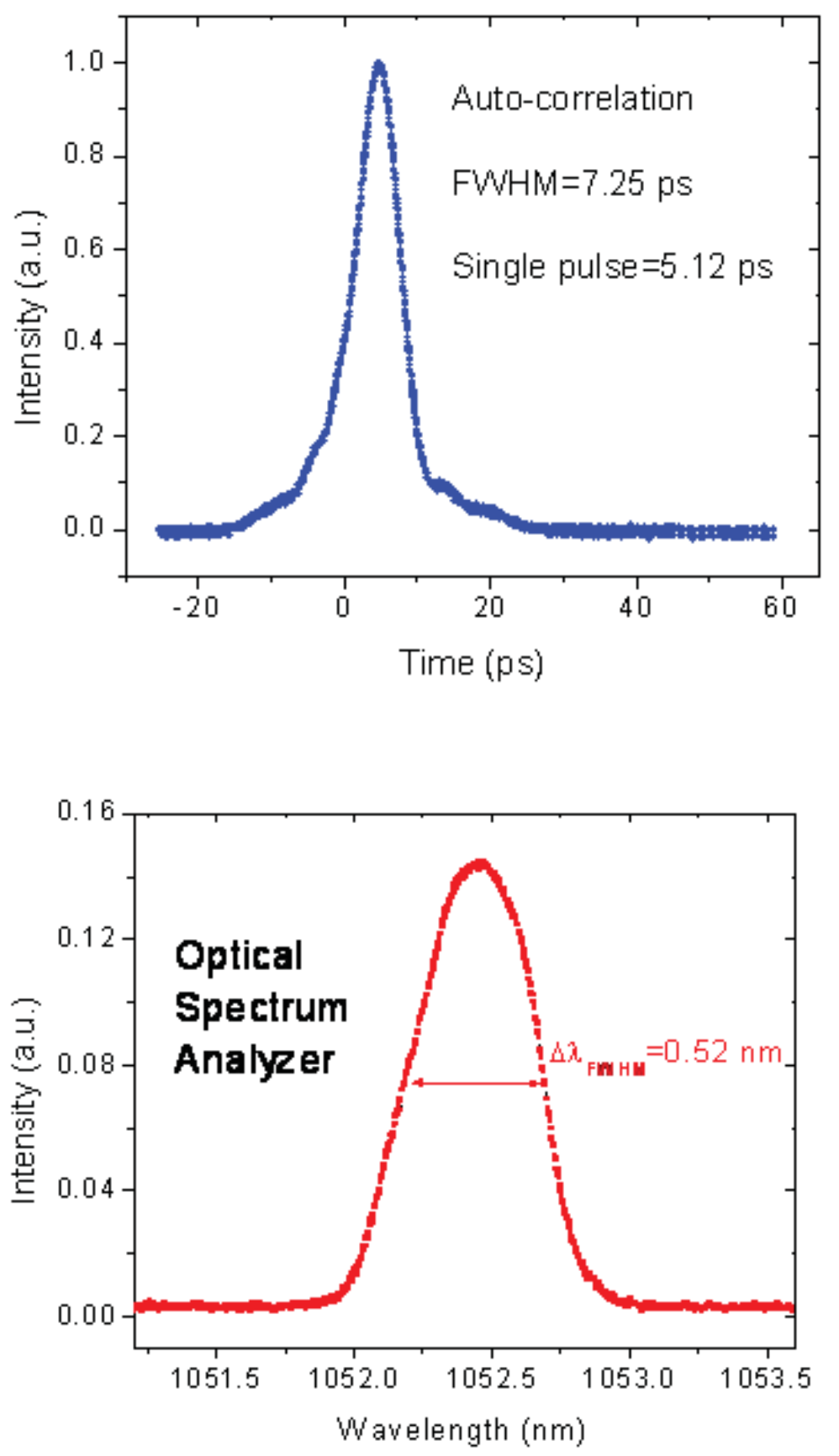

Figure 2.4: Autocorrelation as measured with CWAC (top) and spectrum of the seed laser as measured with Optical Spectrum Analyzer (bottom). The pulse length FWHM was determined to be 7.23 ps, which implies an IR pulse length of 5.12 ps. The bandwidth FWHM was determined to be $0.52 \mathrm{~nm}$. 


\subsection{Seed Laser}

The new seed laser [24] (see Fig. 2.2) provides pulses with a duration of 5 ps, and therefore there is no need to chirp and stretch the pulse any more. As a result, both the long fiber and pulse compressor present in the original configuration were bypassed.

The seed pulse was characterized in both the time and frequency domains and its output was monitored in real time to ensure that the best seed was injected into the amplifiers. The pulse width in the time domain was measured with a rotatingarm continuous-wave autocorrelator (CWAC) [28] [29]. A 50/50 beamsplitter sends light to a fixed delay arm and a variable delay arm, which consists of a flat mirror and a retro-reflector, mounted on a platform spinning at $13.5 \mathrm{~Hz}$. The beams from the two arms center off axis in a focusing lens, and cross each other at the focus in a thin lithium iodate $\left(\mathrm{LiIO}_{3}\right)$ crystal. The crossing angle of the two beams allows phase matched non-collinear second harmonic generation. The resultant green signal is detected with a photomultiplier tube (PMT) and displayed on a digital oscilloscope. A typical autocorrelation profile measured with the CWAC is shown in Fig. 2.4. Assuming a Gaussian waveform for the IR pulse, the FWHM of the autocorrelation yields the single pulse length of $5.12 \mathrm{ps}$, in close agreement with the manufacturer's specifications [24].

A commercial Optical Spectrum Analyzer (OSA) was used to monitor the spectrum of the seed laser [30]. This is a scanning grating monochromator, designed for a resolution of 0.08 to $0.1 \mathrm{~nm}$. The seed laser was coupled into the OSA through a multi-mode fiber approximately $1 \mathrm{~m}$ long. The spectral waveform can be read over GPIB and recorded by computer. A spectrum of the seed laser with FWHM of $0.52 \mathrm{~nm}$ is shown Fig. 2.4.

Another important issue is the timing jitter between the seed pulse and the 
rf signal that feeds into the rf gun and accelerating cavity. The seed laser cannot run independently and must be locked to the rf system. In order to reduce the timing fluctuations in the seed laser, an electronic timing box was provided by the manufacturer so that the phase of the seed pulse is shifted relative to the 81.25 MHz reference signal derived from the master oscillator that also controls the $1.3 \mathrm{GHz}$ rf. The timing box gives a readout of the timing jitter in real time, which was less than $0.2 \mathrm{ps}$ upon daily operation. This timing jitter corresponds to a phase fluctuation of 0.094 degree between the laser pulse and the rf signal, which in practice is completely negligible. The block-diagram of the laser timing chain needed to generate the pulse train and control the optics is shown in Fig. 2.5.

The seed pulse needs to be integrated into the laser system, as shown in Fig. 2.1. The seed laser was located beside the multi-pass amplifier. After traversing the Pulse Picker (PP) and Faraday Rotator (FR), the seed laser pulse was coupled into the multi-pass cavity by a lens of a focal length $60 \mathrm{~cm}$. The pulses rejected by the $\mathrm{PP}$ are directed to the diagnostic table, on which both the CW autocorrelator (CWAC) and Optical Spectrum Analyzer (OSA) are located. The output from the multi-pass amplifier is amplified further by two 2-pass amplifiers. After that, the amplified pulse is sent to the next optical table for frequency doubling and quadrupling and pulse manipulation prior to reaching the photocathode.

Because the conversion efficiency of the crystals is overwhelmingly dependant on the quality of the wavefront, it is very important to maintain the flat wave front in the IR beam and make sure that the beam waist is properly relayed to the crystals. In addition, the UV beam after the crystals needs to be reshaped and relayed to the photocathode, which is about 20 meters away from the laser room.

In the design of the system, we set the reference point of the optical beam at 


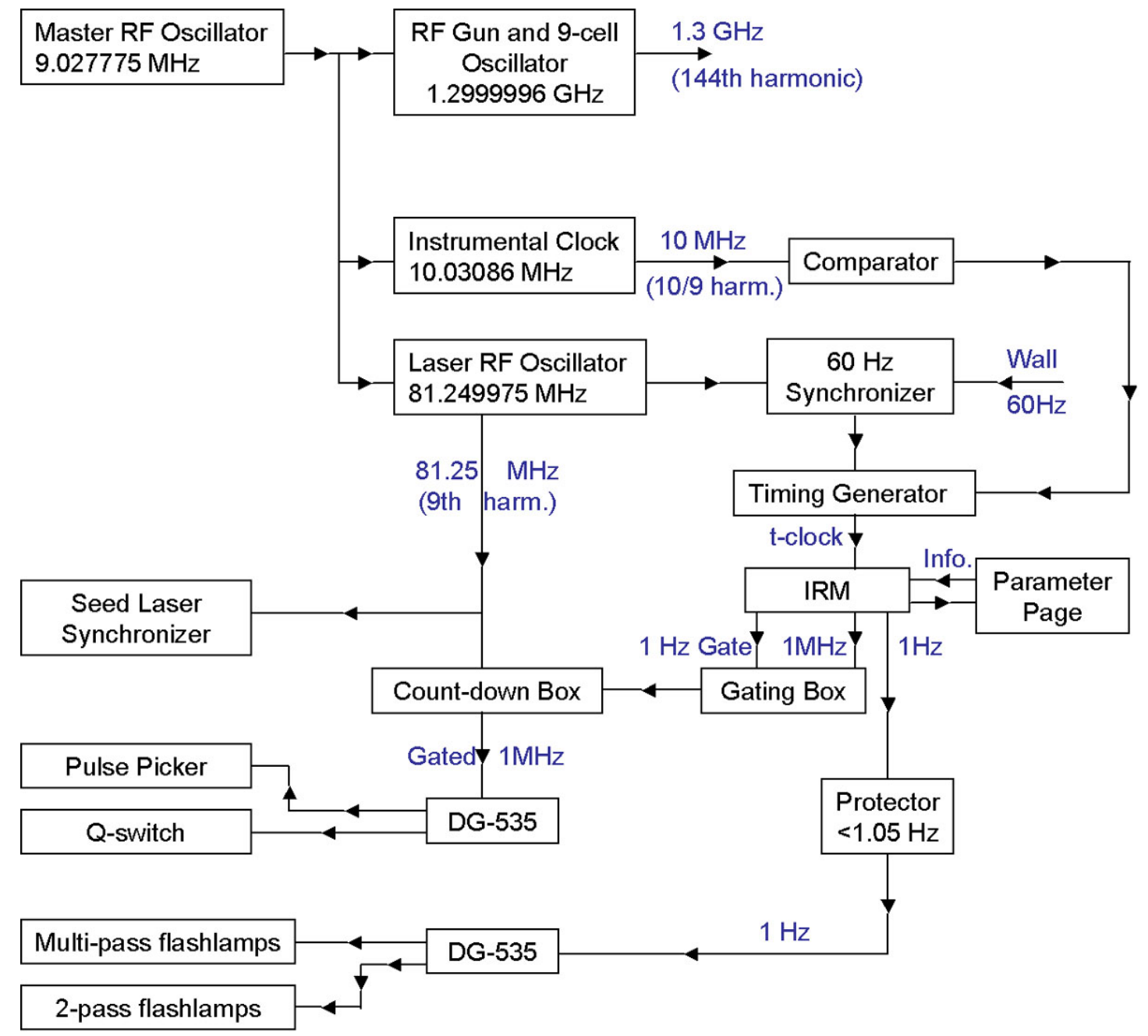

Figure 2.5: Block-diagram of the laser timing system. 
the flat mirror in the multi-pass amplifier. The explicit structure of the multipass cavity is shown in Fig. 2.6. It is a resonator, in which the seed pulse is captured by turning on the Q-switch Pockel cell [31] and makes a certain number of passes through the amplifying medium before it is kicked out by turning off the Q-switch Pockel cell. When the amplifier works in the saturation regime, it is called a "regenerative amplifier". However, because of our requirement of amplifying up to 800 pulses to nearly the same energy, it is necessary to operate in an unsaturated mode. In this way we avoid extracting too much of the stored amplifier energy with each pulse, which would, of course, reduce the gain for the remaining pulses. We use the term "multi-pass amplifier" to indicate operation in the unsaturated regime [32].

The stable resonator cavity is formed by one flat $\left(R_{1}=\infty\right)$ and one curved $\left(R_{2}=5 \mathrm{~m}\right)$ mirror spaced $1.44 \mathrm{~m}$ apart; the optical cavity length is $157.4 \mathrm{~cm}$. For these parameters, the eigenmode of the cavity determines the size of the beam waist on the flat mirror, which is calculated to be $\sim 0.88 \mathrm{~mm}$.

In the first step of the alignment, the output from the seed laser must be coupled efficiently into the resonator cavity. Namely, the beam waist of the seed laser must be relayed onto the flat mirror in the multi-pass cavity with appropriate magnification to match the eigenmode diameter. From the product report provided by the manufacturer, the beam waist is located inside the exit window of the seed laser at a distance of $50 \mathrm{~cm}$ and the size of the beam waist there is about $0.25 \mathrm{~mm}$. A lens with focal length of $60 \mathrm{~cm}$ placed about $10 \mathrm{~cm}$ away from the exit window is adequate and resulted in good coupling of the seed pulse into the amplifier cavity.

It should be noted that the transverse profile of the seed laser was elongated in the horizontal dimension due to the fact that the Nd:YLF crystal, the gain 


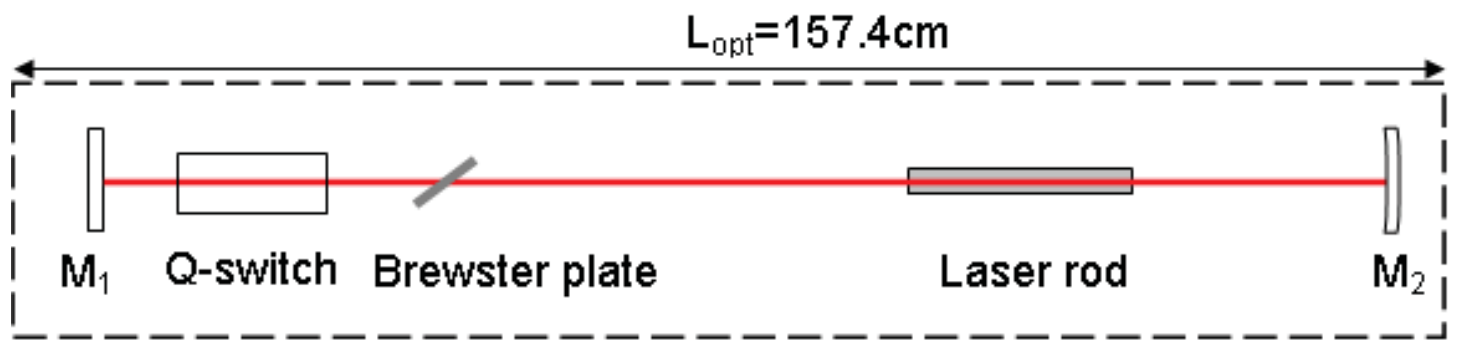

Figure 2.6: Schematic top view of multi-pass cavity. M1 and M2 are the flat and the curved mirrors respectively. The Q-switch and Brewster plate act as an input/output device.

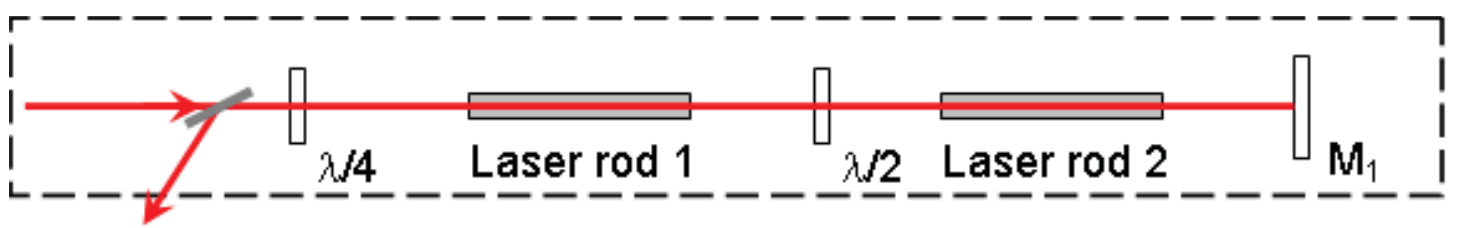

Figure 2.7: Schematic top view of the two 2-pass amplifiers. A half-wave plate between the rods is used to balance the birefringence of the rods. 
medium of the seed laser, was cut at Brewster's angle to minimize the unwanted polarization in the output. Since the multi-pass cavity was designed for a homogeneous transverse profile, it was necessary to compensate the horizontal distortion with an anamorphic prism. The beam profile before and after the prism is shown in Fig. 2.3.

\subsection{Amplification and Conversion to UV}

After propagating through the lens, PP, FR, half waveplate, Brewster plate and mirrors, the $5.5 \mathrm{~nJ}$ seed laser pulse (decreased to $\sim 4 \mathrm{~nJ}$ ) was injected into the multi-pass cavity by the Brewster plate (see Fig.2.6) with vertical polarization. The pulse passes through the Q-switch Pockels cell [31] that is statically biased for quarter wave, acquires a quarter-wave retardation, is reflected from the flat end mirror, and passes again through the Q-switch acquiring a further quarter wave retardation to horizontal polarization. With this polarization the pulse goes through the Brewster plate and continues through the cavity, and after one round trip through the Nd:glass rod returns to the Q-switch. At this point in time, the Q-switch has been triggered on to zero-wave retardation so that the pulse is trapped inside the cavity. The voltage on the Q-switch is held high for the length of time necessary for the desired number of round trips. After that it is triggered back to the quarter-wave retardation so that the polarization of the pulse is flipped up and the pulse is kicked out by the Brewster plate. The Faraday isolator (with permanent magnets) is used to separate the output pulse, and directs it to the 2-pass amplifiers.

The seed pulse grows rapidly in the resonator by traveling back and forth through the laser rod during the time window set by the Q-switch Pockel's cell. 

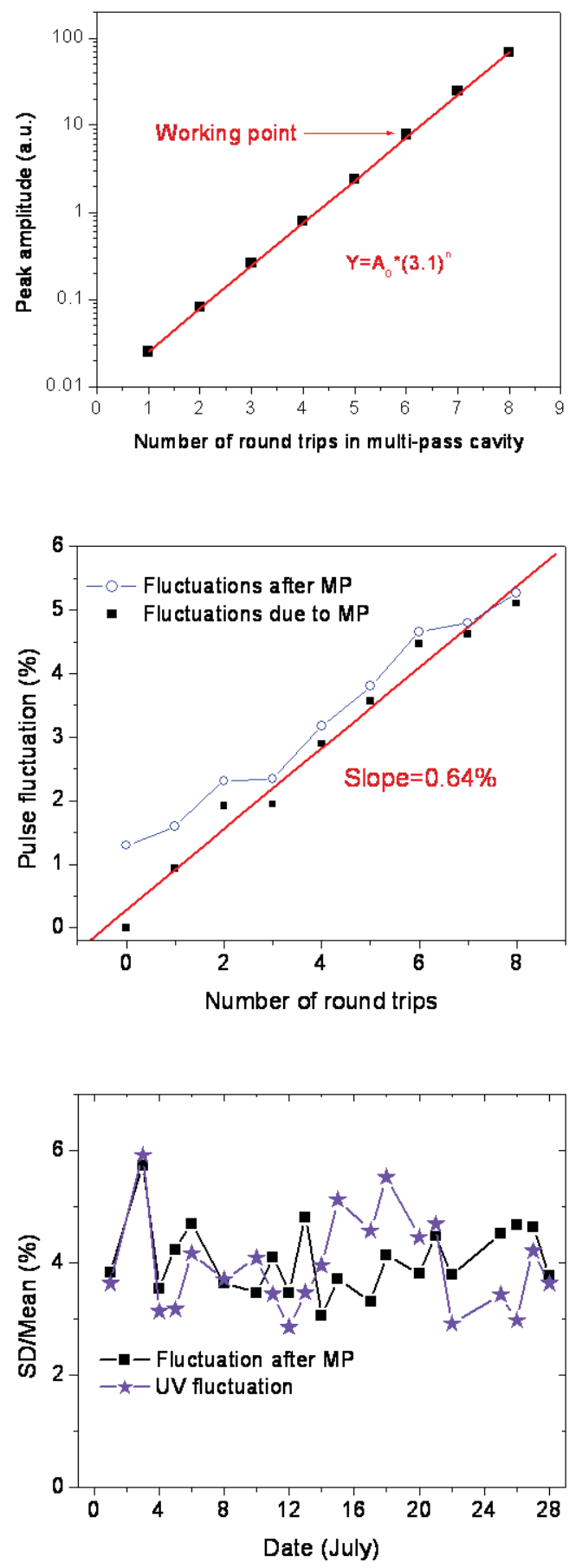

Figure 2.8: Single pulse amplitude vs. the number of round trips in the multi-pass cavity (top). Shot to shot fluctuations after the multi-pass amplifier measured for a different number of round trips (middle). Shot to shot fluctuations after the multi-pass amplifier and after frequency conversion to UV for the entire month of July 2005 (bottom). 
Fig. 2.8 (top) shows the single pulse amplitudes measured with a fast photodiode versus number of round trips. There are several conclusions that can be drawn from these data. First, the pulse amplitude exhibits a consistent exponential growth with an amplification factor of 3.1 for each single round trip. Second, the amplification of the pulse does not show any saturation up to 8 round trips. Third, the fact that the amplification of the pulse is evenly distributed on different round trips implies that the seed pulse was efficiently coupled into the multi-pass cavity and that the multi-pass cavity was well aligned. The overall energy of the single pulse after 6 round trips was measured at $6 \mu \mathrm{J}$, which represents a total gain of 1500 in the multi-pass amplifier. Since, as discussed below, the fluctuations of the pulse amplitude are dominated by the power instability of the pumping light, a small number of round trips is desired. Because the pulse will be amplified further by the two 2-pass amplifiers, we set the time window of the Q-switch in our daily operation for 6 round trips ( $\sim 52 \mathrm{~ns})$.

After exiting the multi-pass amplifier, the IR pulse is coupled by the telescope system into the two 2-pass amplifiers which consist of two balanced laser rods (see Fig 2.7). The single pulse energy is multiplied by more than 25 times to $\sim 150 \mu \mathrm{J}$. The telescope consists of two $1 \mathrm{~m}$ focal length lenses, the flat end mirror in the multi-pass cavity at the focal plane of the first lens and the reflection mirror in the 2-pass amplifiers at the focal plane of the second lens. The other focal planes of the two lenses coincide at midpoint between the lenses. A spatial filter may be inserted at the midpoint to smooth out the transverse profile after the multipass.

Similarly, another telescope system with two $1 \mathrm{~m}$ lenses located after the twopass amplifier and before the crystals, relays the beam waist to the frequency doubling crystal. We achieved frequency doubling efficiency in excess of $50 \%$ and frequency quadrupling efficiency of up to $40 \%$. The crystal conversion efficiencies 

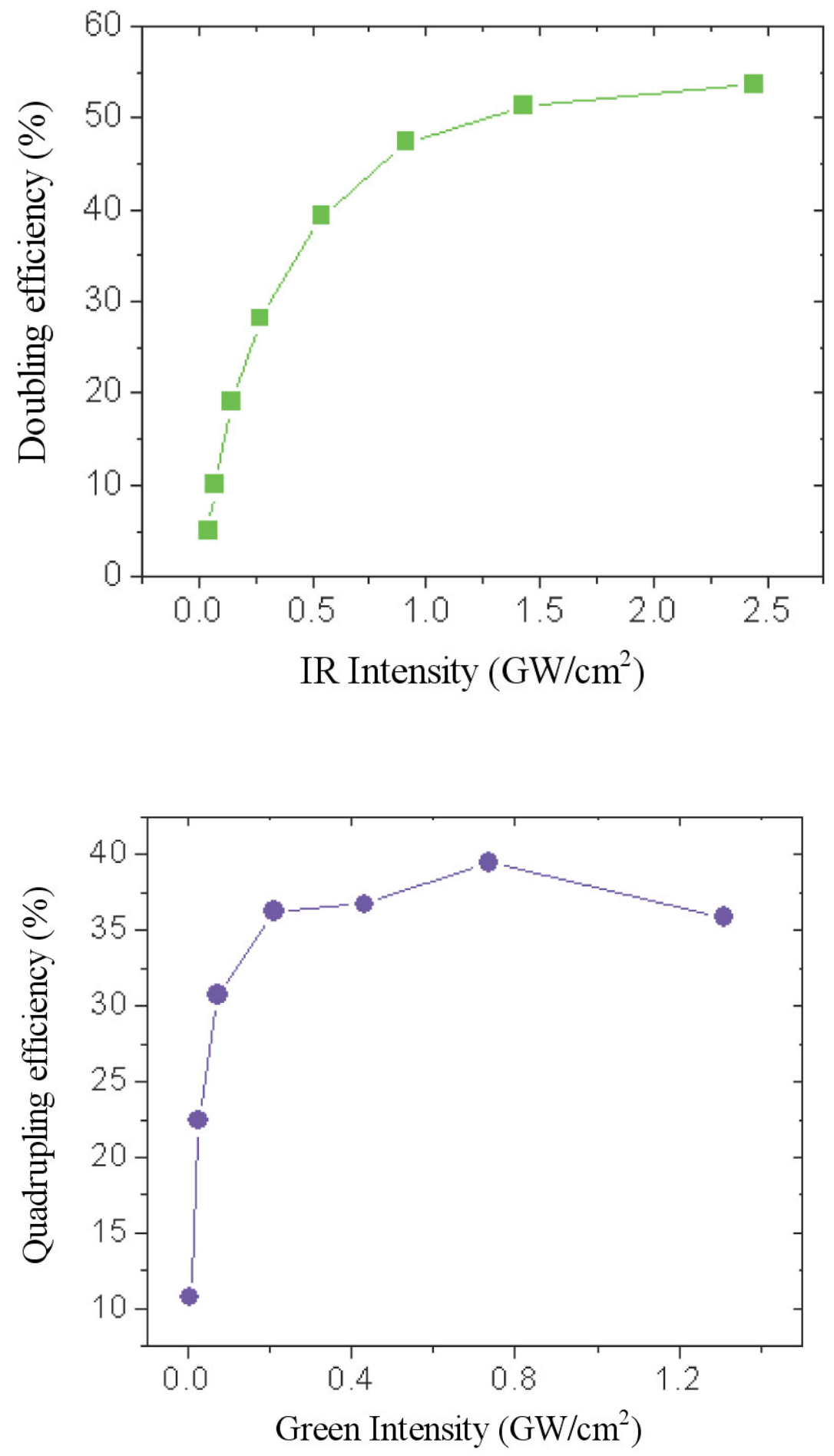

Figure 2.9: Efficiency of the doubling (top) and quadrupling (bottom) crystals for different input pulse intensity. 
measured under different input power are plotted in Fig. 2.9. In Appendix B we discuss a MatLab simulation that we developed to analyze second and fourth harmonic generation. At the maximum input intensity, both crystals are in the deeply saturated region and this helps to reduce the fluctuations in the green and in the UV.

After being converted from IR (1054 nm) to UV (263 nm), the laser beam was directed to a diagnostic "target" by a telescope consisting of two UV lenses with focal lengths of $0.2 \mathrm{~m}$ and $1 \mathrm{~m}$ respectively. The telescope magnifies the beam by five times as indicated in Fig. 2.1. A $50 \mu \mathrm{m}$ spatial filter was inserted at the focal spot between the two lenses to improve the UV transverse profile. This causes an energy loss of $\sim 50 \%$.

The image at the position of the "target" was relayed by an imaging system to the photocathode, which is located $20 \mathrm{~m}$ away from the laser room.

\subsection{System Performance}

After the upgrade was completed, the performance of the entire laser system was checked, including pulse to pulse fluctuations, transverse and longitudinal profile of the UV beam, and the operation of the transport line to the cathode.

\subsubsection{Shot to Shot Fluctuations}

Hereafter, we refer to pulse train to pulse train fluctuation (1 Hz repetition rate) as "shot to shot fluctuation". An important aspect of the laser performance is its repeatability from shot to shot as this determines the charge fluctuations in the electron beam. The single seed pulse after the pulse picker was scrutinized with a fast photodiode. With $1 \mathrm{~Hz}$ repetition rate, the amplitude of more than 
50,000 pulses was recorded and the ratio between the standard deviation and mean was measured to be $1.3 \%$; this represents the fundamental noise originating from the seed laser. The shot to shot fluctuation after the multi-pass amplifier was measured by averaging more than 100 shots and dividing the mean by the standard deviation. The results were recorded for a different number of round trips and are shown in Fig. 2.8 (middle). The open circles are the measured data and the noise for "zero" round trip was set at $1.3 \%$ as determined from the seed laser. To pinpoint the source of the noise, it is necessary to decouple the noise originating in the seed laser and in the multi-pass amplifier. We assume that these two noise sources are independent and can be separated quadratically,

$$
F l u c_{M P}=\sqrt{\text { Fluc }_{\text {Measured }}^{2}-F l u c_{\text {Laser }}^{2}}
$$

Here, $F l u c_{M P}$ are the amplitude fluctuations contributed solely by the multi-pass

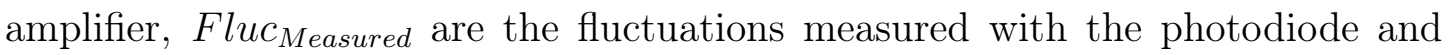
Fluc $_{\text {Laser }}$ is the seed laser fluctuation (1.3\%).

The squares shown in Fig. 2.8 (middle) represent the result of such a separation. They were fit by a straight line with a slope of $0.64 \%$, which implies that each round trip adds $0.64 \%$ to the amplitude fluctuation in the final output. This level of single round trip fluctuation can be explained by the voltage instability of the power supply for the multi-pass amplifier flash lamps. According to our measurement, the voltage fluctuation of the power supply is less than $0.2 \%$; this leads to fluctuations in power of $<0.4 \%$. By assuming a uniform pumping efficiency in this short period of time ( $\sim 52 \mathrm{~ns})$, one obtains sub $0.8 \%$ fluctuation for each round trip, consistent with the measured fluctuations. It is then reasonable to conclude that the noise of the multi-pass amplifier is dominated by the noise in the power supply.

After the multi-pass amplifier, the pulse was coupled to the 2-pass amplifiers 

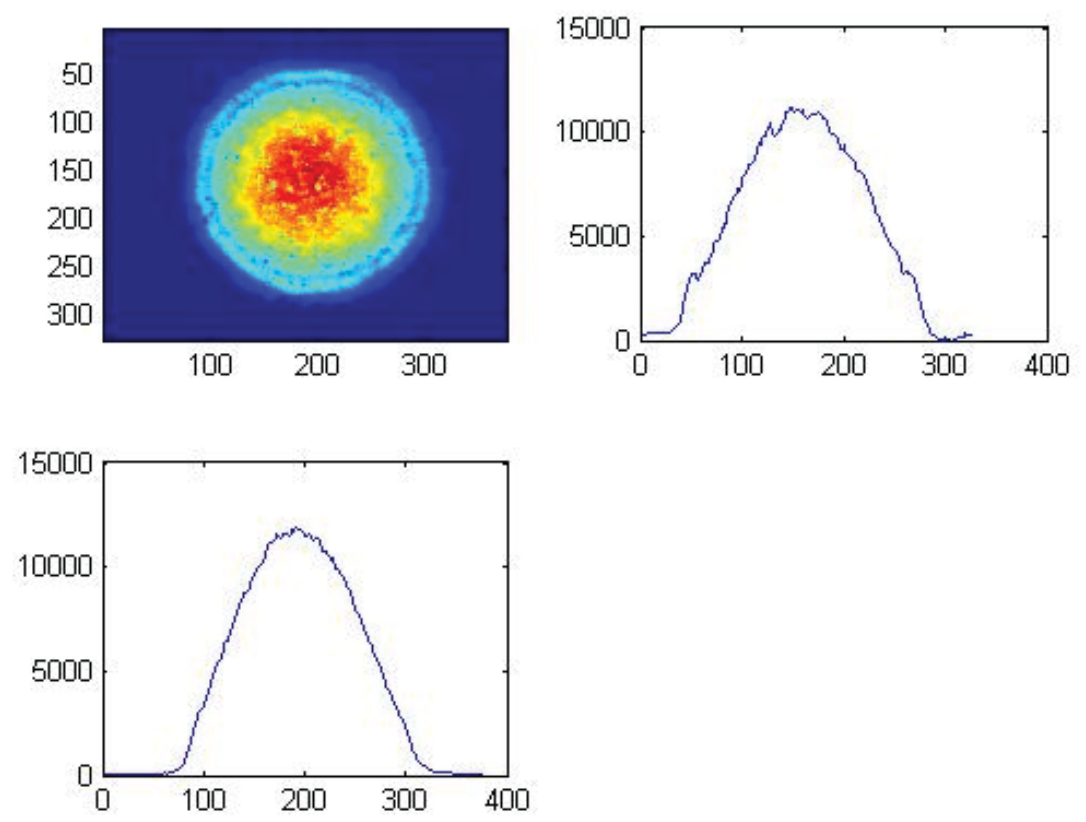

Figure 2.10: UV beam profile measured on the target in the laser room and its projections. A $50 \mu \mathrm{m}$ spatial filter was inserted to improve the UV transverse profile. The beam size is about $4.3 \mathrm{~mm}$ FWHM.
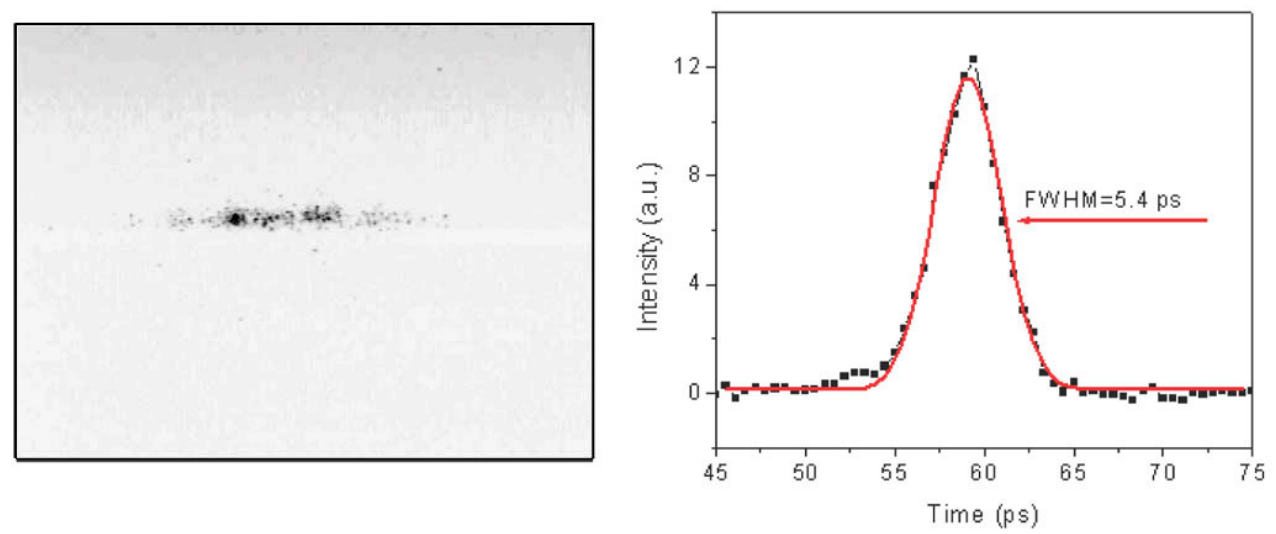

Figure 2.11: Picture of a single UV pulse recorded by the streak camera (left). Projection of the image on the vertical axis (right), and Gaussian fit to the data points. 
and no significant increase of the fluctuations was observed. The reason is that the multi-pass amplifier and 2-pass amplifiers are independent of each other so their noise contributions add quadratically. Furthermore, in each 2-pass amplifier (they use similar power supplies as the multi-pass amplifier), the pulse experiences only one round trip, which adds negligible noise to the final output.

The shot to shot fluctuations were monitored at all stages along the beam path. They were measured after the multi-pass amplifier, after the 2-pass amplifier, after the frequency doubling and quadrupling crystals. This is shown in Fig. 2.8 (bottom), where the fluctuations of the pulse amplitude after the multipass amplifier and after the UV crystal, as measured for the entire month of July, 2005 are plotted on the same graph. As can be seen, the amplitude fluctuations measured of the UV output follow closely the fluctuations after the multi-pass amplifier. We conclude that the multi-pass amplifier is the dominant noise source in the entire laser system. Reduction of the shot to shot fluctuations beyond the present level could possibly be achieved by replacing the multi-pass amplifier with a diode pumped system.

\subsubsection{Transverse and longitudinal profiles}

The transverse uniformity of the laser beam on the photocathode is crucial for generating a high quality electron beam with low emittance [33]. Since a UV imaging system that relays the image in the laser room to the photocathode is in place, it suffices to measure the beam quality on the diagnostic "target" in the laser room. The UV beam after the quadrupling crystal is directed by the mirrors and the telescope to the "target" as shown in Fig. 2.1 (bottom). Fig. 2.10 shows the UV image on the "target" taken with a CCD camera. The beam profile is close to a Gaussian and has a FWHM of $4.3 \mathrm{~mm}$, which is well suited for most 
applications. In case a smaller beam size is desired, an iris can be inserted at the location of the "target" to reduce the beam size.

The longitudinal profile of a single UV pulse was measured with the streak camera which has a time resolution of $\sim 2$ ps. No pulse length fluctuations were observed and a typical picture of the longitudinal profile of a single UV pulse obtained by the streak camera is shown in Fig. 2.11. The UV pulse length is determined to be $5.4 \mathrm{ps}$, almost identical to the IR seed pulse length. This can be understood by the fact that both the doubling and quadrupling crystals are operating in the deep saturation regions, and therefore no pulse narrowing should be expected from either conversion process.

\subsubsection{Long pulse train}

As mentioned, the laser system is able to deliver up to 800 pulses. A long train consisting of 400 seed pulses after the pulse picker is shown in Fig. 2.12 (top). Because of the stable output of the seed laser, the train envelope is extremely flat. However, after the multi-pass and 2-pass amplifiers, the shape of the pulse train is distorted. This is shown in the middle panel of Fig. 2.12. Although the rising part of the pulse train is very fast and sharp, the decay is somewhat slow and the amplitude of the last 80 pulses is reduced. To achieve a flat top long train, a pre-shaping Pockels cell can be inserted in the seed pulse line before the multi-pass amplifier so that the seed pulse train is shaped such that the losses in the amplification process are exactly compensated [21].

In the bottom panel of Fig. 2.12 we show a pulse train of $100 \mathrm{UV}$ pulses on the photocathode captured with a fast digital oscilloscope (green) and corresponding electron bunch train (blue). 


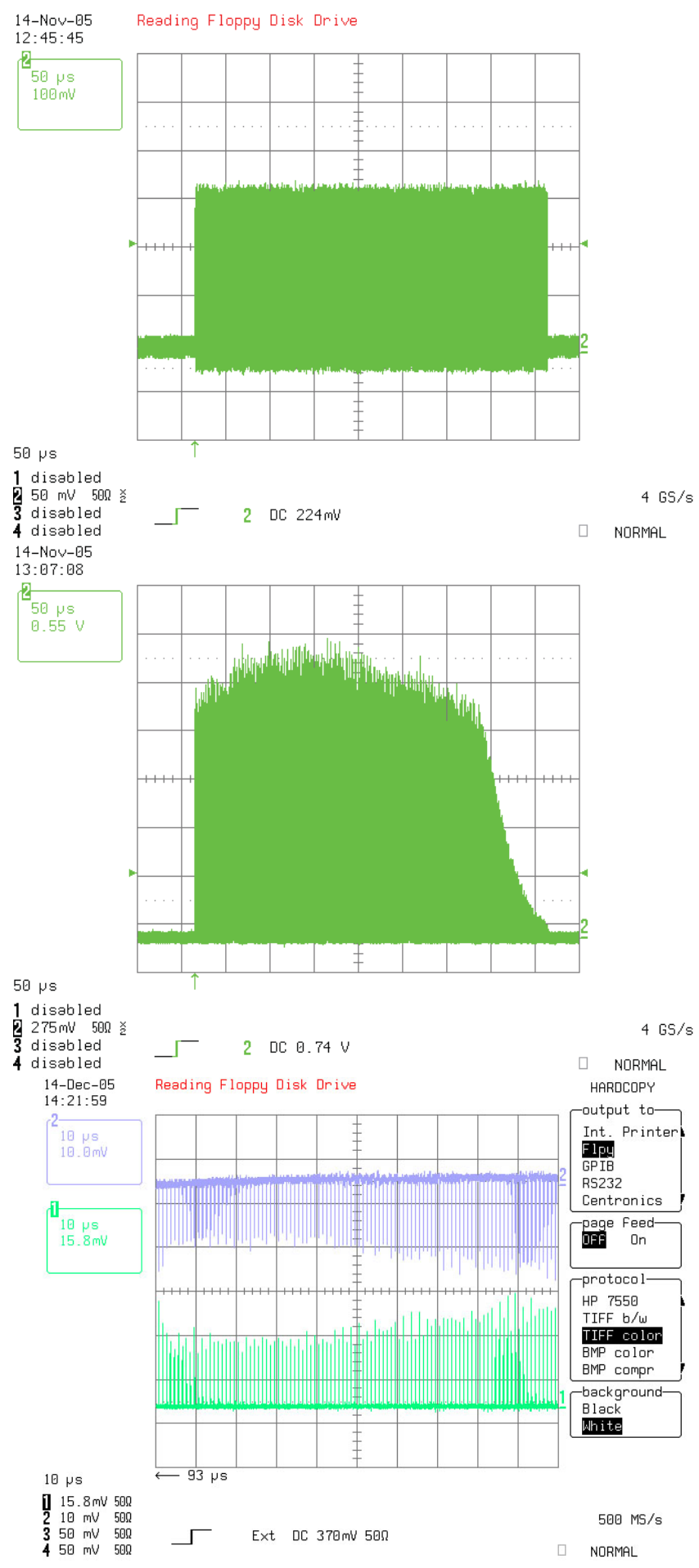

Figure 2.12: A long pulse train of 400 seed (IR) pulses captured with a fast digital oscilloscope (top); the same long pulse train after the 2-pass amplifiers (middle). And (bottom) a pulse train of $100 \mathrm{UV}$ pulses captured with a fast digital oscilloscope (green); the corresponding electron bunch train (blue). 


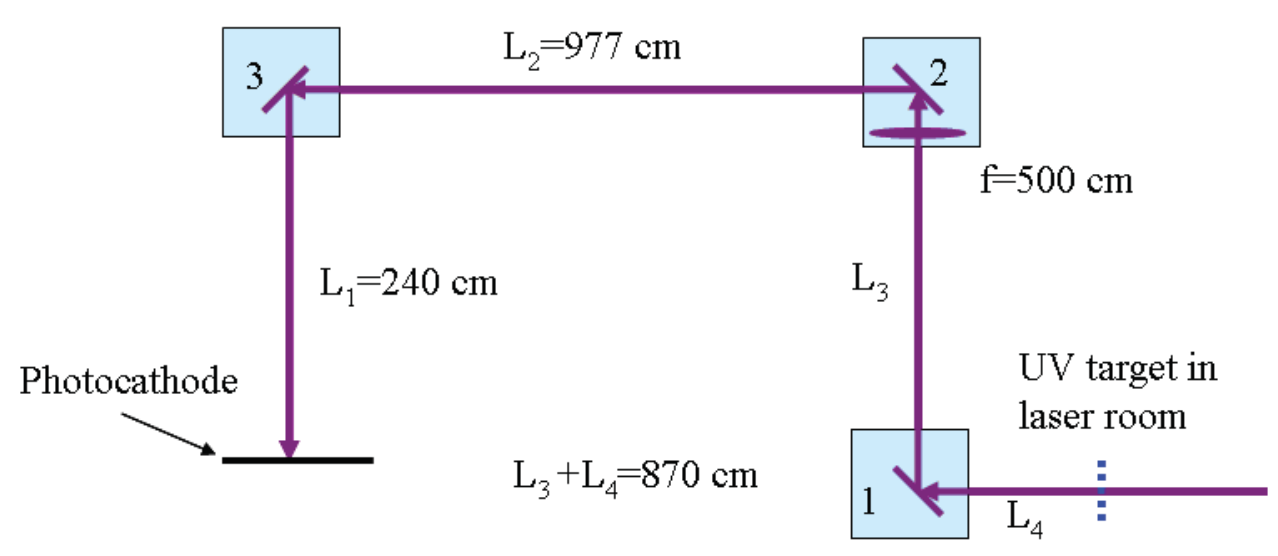

Figure 2.13: Schematic diagram of the UV beam transport line.

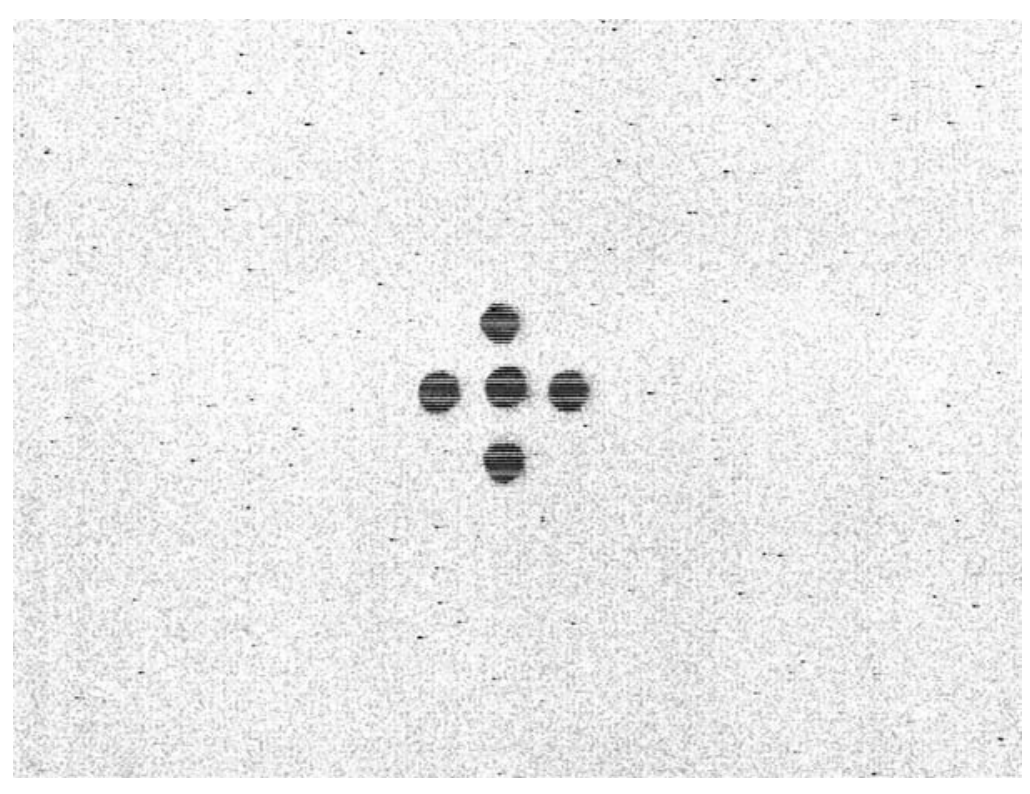

Figure 2.14: Five beamlets picture observed on the photocathode after the UV imaging relay system. 


\subsubsection{Transport to the cathode}

To relay the UV beam in the laser room onto the photocathode which is located more than $20 \mathrm{~m}$ away, an imaging system was installed along the beam transport line. The beam transport line is folded and there are only three turning corners, where the optics can be mounted in the boxes. Fig. 2.13 shows the schematic of the transport beam line (as a 2-dimensional projection). A UV coated standard singlet spherical plano-covex lens with focal length of $5 \mathrm{~m}$ was inserted in front of the flat mirror in turning box 2 . With this imaging system we can introduce a mask in the laser room and then image its pattern onto photocathode. In particular, we can study the evolution of the non-uniform transverse distribution of the electron beam and benchmark the simulation code of the electron beam line $[36]$.

A five-beamlet mask made by drilling five $0.7 \mathrm{~mm}$ holes in a brass plate was inserted in the laser room and a high-quality image was obtained on the photocathode in the cave (20 m away) as shown Fig. 2.14. An electron bunch with the corresponding transverse distribution $(0.44 \mathrm{nC}$ charge) was accelerated to an energy of $15.7 \mathrm{MeV}$. We then observed the transverse distribution on the optical transition radiation (OTR) screen for different currents of the solenoid lens. The OTR screen was made of yttrium aluminum garnet (YAG) and located $4.6 \mathrm{~m}$ downstream of the photocathode. The observed distributions were compared with simulation performed by a computer program Impact [37] which incorporates a three dimensional space charge algorithm. Such an algorithm is needed for calculating the beam dynamics associated with beams with no cylindrical symmetry. An example of a set of pictures for the solenoid current settings of 237 A, 209 A, 199 A, 188 A, 182 A, and $173 \mathrm{~A}$ is presented in Fig. 2.15. 

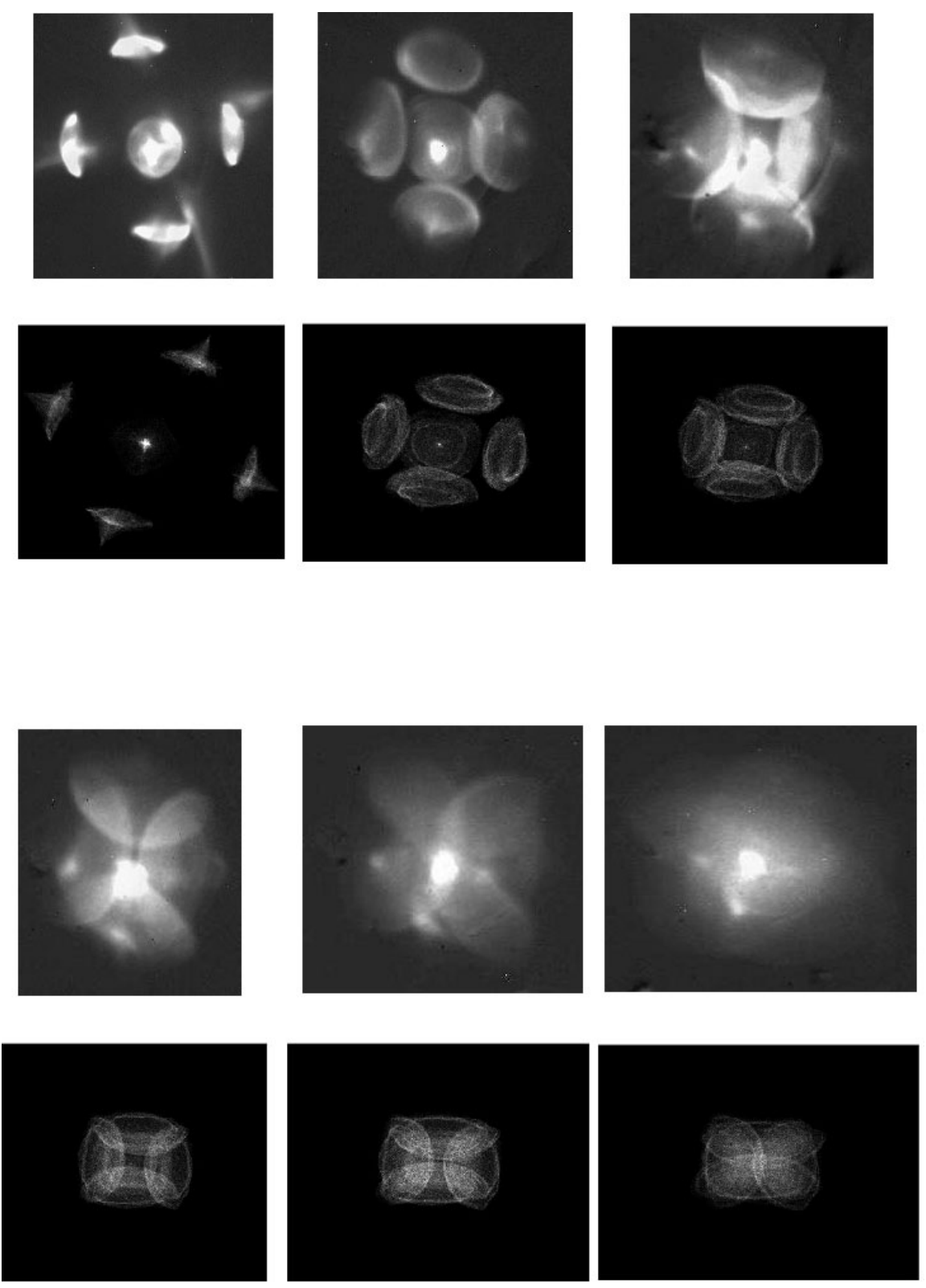

Figure 2.15: Dependence of the transverse electron bunch image on the solenoid current. In each pair of rows, the upper is experimental and the lower is simulation. The currents on the primary solenoid are, from left to right, 237 A, 209 A, 199 A (top pair of rows), 188 A, $182 \mathrm{~A}$, and $173 \mathrm{~A}$ (bottom pair); the images are not to scale. 


\section{Chapter 3}

\section{Manipulation of the Longitudinal}

\section{Profile}

In this chapter we present studies of transverse emittances and electron beam pulse length for various operating points of the electron source: electron beam charge, laser length and spot size, and solenoid settings. We especially study the impact, on transverse emittance, of short Gaussian versus long square temporal distributions of the photocathode drive-laser.

Optimization of the UV laser pulse shape on the cathode should be relevant in reducing the space charge effects. The simplest way to reduce the charge density is to increase the laser spot size, but this results in increased emittance. A more effective way would be to elongate the laser pulse, while also making its longitudinal profile square. One of the most efficient shapes for reducing the space charge effect is a "can" shape", which has a square profile in both the

\footnotetext{
${ }^{1}$ An ellipsoid of revolution is the best possible shape since, ideally it provides no emittance growth due to the space charge effect. A laser pulse of such shape, however, is very challenging to obtain.
} 


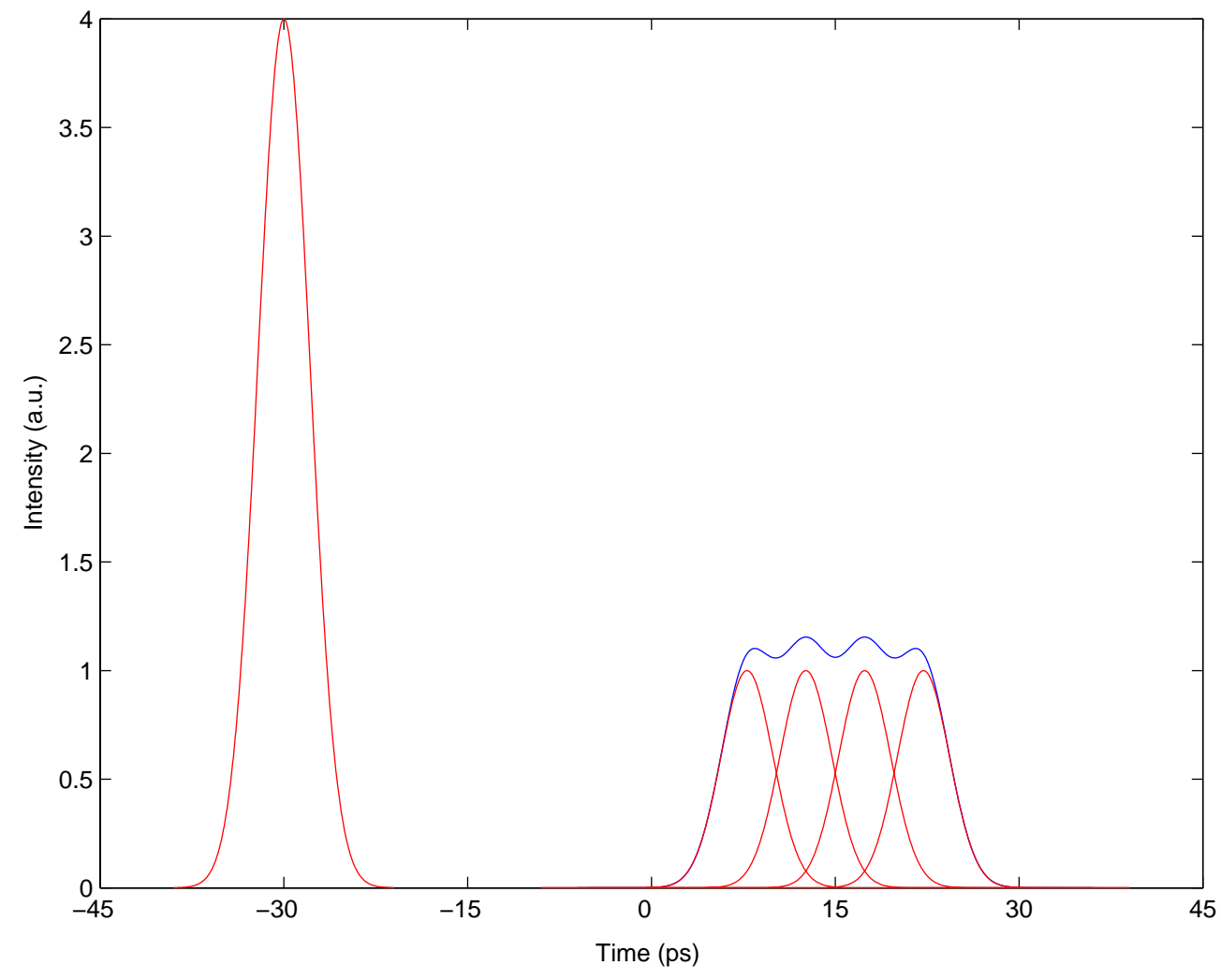

Figure 3.1: The pulse-stacker principle of operation. A pulse-stacker takes a single Gaussian (left); splits it into four beams, and stacks the beams after introducing a delay between them (right). The four stacked Gaussians form a quasi flat-top distribution (blue). 
transverse and longitudinal domains. In the transverse domain a quasi-square shape is relatively easy to achieve by introducing an iris in the laser beam and imaging the iris onto the cathode. However, in the longitudinal domain this is not such an easy task. To generate a long and flat-topped temporal distribution we use a "pulse stacker". In this device the photoinjector laser beam is split into four different beams which are then recombined (stacked) after a time delay is introduced between the pulses. This results in a quasi flattop pulse as shown in Fig. 3.1.

\subsection{Pulse Stacker}

We use a modified DESY-type pulse stacker designed by S. Schreiber [35] (see Fig. 3.2). A Gaussian laser pulse is split in two by a prism (Pz) so that the $\mathrm{P}-$ polarized beam goes straight and the S-polarized beam is reflected sideways. Each of the beams ( $\mathrm{P}$ and $\mathrm{S}$ ) hit the set of mirrors $(\mathrm{M})$ and then they are recombined by another prism $(\mathrm{Pz})$; one set of mirrors is positioned on a movable platform so that the delay between the two beams can be adjusted. After the first stage of splitting and recombining the double Gaussian goes through a second stage analogous to the one described above. Half-wave plates $(\lambda / 2)$ are used to adjust the relative intensities of the stacked Gaussians; intensity adjustments along with the adjustable delay enable us to optimize the flattop shape. Each stacking stage makes two copies of the input and then stacks them; so, in the most general case when we have $n$ stacking stages the final number of copies utilized is $2^{n}$.

One of the features of this design is that the copies utilized by each stacking stage have different polarization. Thus we are able to avoid the longitudinal interference between adjacent pulses by use of the alternate polarization scheme: 
SPSP. Another feature is that all the input intensity appears in the stacked pulse (in older designs half of the light was lost [29]).

The pulse stacker has been successfully built and commissioned at A0. The use of the imaging system reduces the transverse fluctuation of the stacked beam spot centroids with the respect to each other, when they reach the cathode. The resulting quasi-flattop pulse length is 20.8 ps full width half $\max$ (FWHM) which as expected, is four times longer than the original single Gaussian (see Fig. 3.3). The overall transmission through the pulse stacker is about $80 \%$, the intensity loss is due to the fact that the laser beam encounters sixteen surfaces while passing through the pulse stacker. The loss could be slightly reduced by substituting Brewster's plates for the prisms.

The stacked pulse "flatness" (the variation of the intensity in the flattop region) is better than $5 \% \mathrm{rms}$. The rise time $(10 \%-90 \%)$ is $6.4 \mathrm{ps}$; of course, the rise-time is completely determined by the original Gaussian pulse shape. In order to make the pulse more square, that is to decrease the ratio of the rise-time over FWHM, we have been considering adding one more stacking stage, thus increasing the FWHM by a factor of two. The flattop pulse length depends on the number of pulses stacked and on the delay between them; the overall pulse length can be varied from 8 to 25 ps FWHM, by stacking two or four pulses. It is not advisable to have more than three stacking stages since the pulse length must be much smaller than the rf period, which is 769 ps; otherwise the energy spread increases resulting in increased longitudinal emittance (the longitudinal emittance is proportional to the cube of the pulse length [41]). 


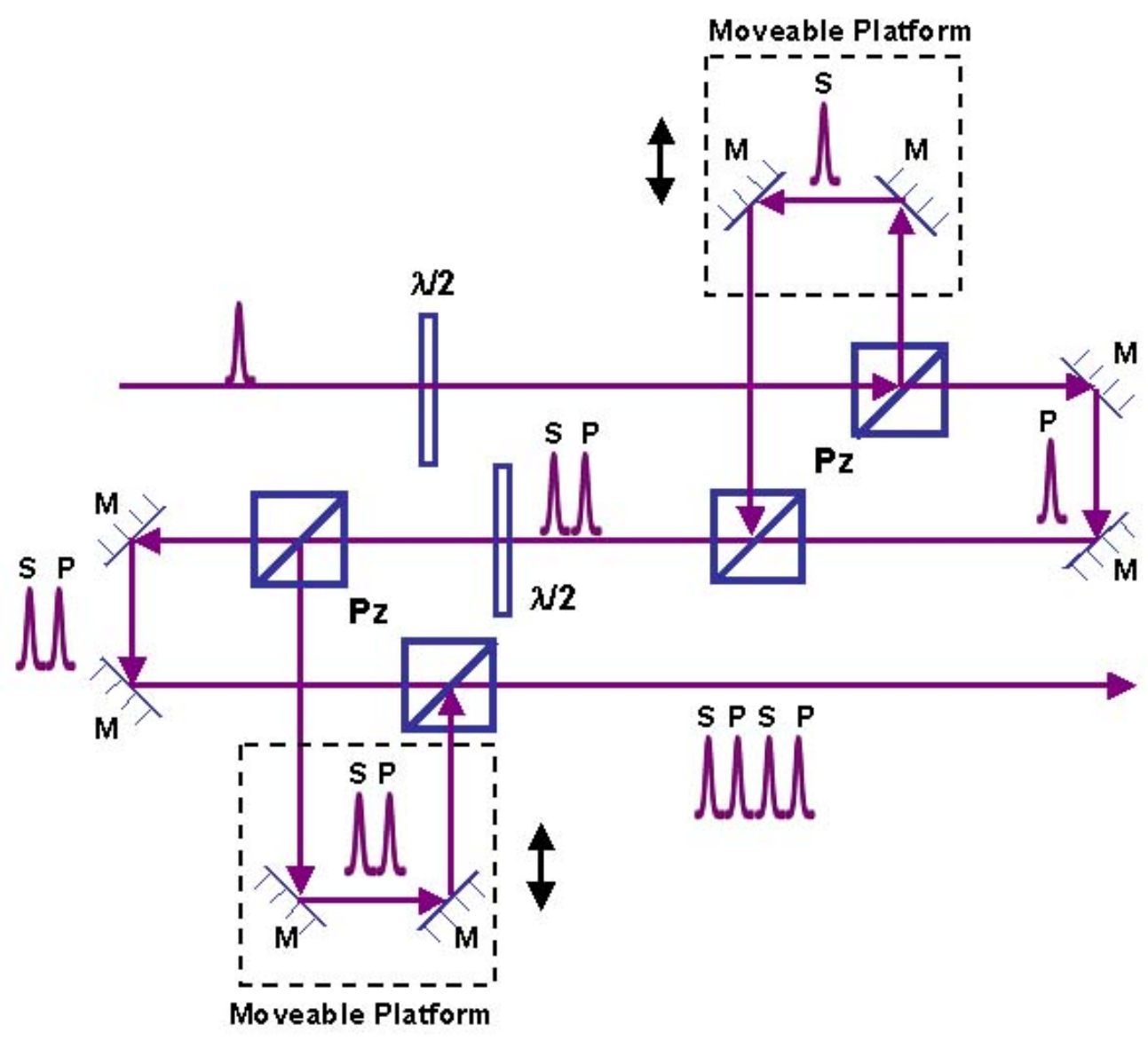

Figure 3.2: Pulse Stacker; M-mirror, $\lambda / 2$-halfwave plate, Pz-prism, SPalternating polarizations. 

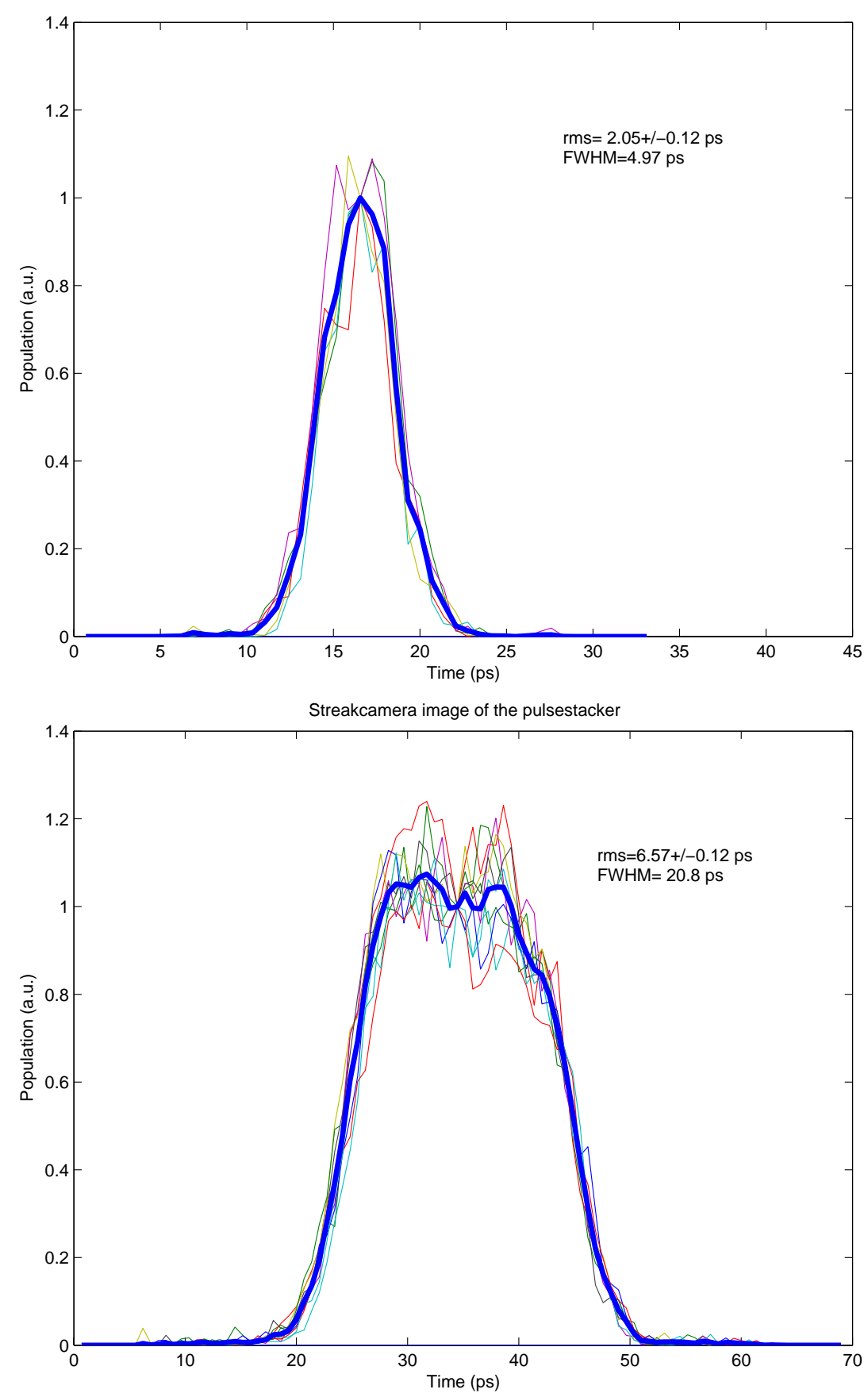

Figure 3.3: Time profile of the UV laser pulse measured by a streak camera. Five Gaussian (top) and eight flat-topped (bottom) laser shots and their average (thick blue). 


\subsection{Emittance}

\subsubsection{Introduction}

An ensemble of particles can be fully described in a six-dimensional phase space of position and canonical momentum $(\vec{r}, \vec{p})$. According to Liouville's theorem, the volume of an ensemble of non-interacting particles in such phase space is invariant. There is a set of sub-spaces $\mathcal{G}_{i}=\left(r_{i}, p_{i}\right)$ associated with each physical dimension $(i=x, y, z)$. It follows that in the case of weak mutual coupling between dimensions, which is usually a true approximation, the area of a two-dimensional phase space associated with a particular sub-space remains constant.

The normalized emittance of the sub-space is defined as:

$$
\epsilon_{n, x} \equiv \frac{\Gamma^{x}}{\pi m c},
$$

where $\Gamma^{x}$ is the area the ensemble occupies in the $\left(x, p_{x}\right)$ sub-space, $m$ is the mass of an electron, and $c$ is the speed of light.

The statistical definition of emittance is

$$
\epsilon_{n, x}^{r m s} \equiv \frac{1}{m c} \sqrt{\left\langle x^{2}\right\rangle\left\langle p_{x}^{2}\right\rangle-\left\langle x p_{x}\right\rangle^{2}} .
$$

And in the limit of the paraxial approximation $\left(p_{x, y} / p_{z} \ll 1\right)$, the transverse emittance can be written as:

$$
\epsilon_{n, x}^{r m s}=\beta \gamma \sqrt{\left\langle x^{2}\right\rangle\left\langle x^{\prime 2}\right\rangle-\left\langle x x^{\prime}\right\rangle^{2}},
$$

where $x^{\prime} \equiv p_{x} / p_{z}$; the sub-space of $\left(x, x^{\prime}\right)$ is referred to as the "trace space".

Emittance is one of the most important characteristics of a beam of charged particles. Along with energy, charge and duration (time structure) of a bunch, emittance characterizes the beam. In the ideal case of particles moving in exactly 
the same direction (no transverse motion) and of an infinitesimal bunch size (zero cross-section) the emittance value in the limit of classical mechanics would be zero.

In the quantum limit the emittance has a non-zero value due to the uncertainty principle. For a single electron the normalized rms emittance is

$$
\epsilon_{n, x}=\frac{\hbar}{m c} \approx 4 \cdot 10^{-13} \mathrm{~m}
$$

or the Compton wavelength of the electron. Here $\hbar$ is Planck's constant. For the six dimensional phase space the volume is given by

$$
\Gamma=\left(\frac{\hbar}{m c}\right)^{3},
$$

Thus for an ensemble of $N$ electrons, the minimum phase space volume the ensemble occupies is expressed as

$$
\Gamma=\left(\frac{\hbar}{m c}\right)^{3} \frac{N}{2},
$$

where the factor of $1 / 2$ is included to account for two possible spin orientation for the electron. Thus for $N$ electrons we have the quantum limited rms emittance,

$$
\epsilon_{n, x}=\left(\frac{N}{2}\right)^{\frac{1}{3}} \frac{\hbar}{m c},
$$

which for $1 \mathrm{nC}\left(N \approx 6 \cdot 10^{9}\right)$ is in the order of $10^{-3} \mathrm{~mm}$-mrad [38]. However, when describing a real world ensemble of electrons we see that a typical value of the emittance is three orders of magnitude greater than the quantum limit. In the following we will consider sources contributing to the growth of the emittance in real beams.

\subsubsection{Sources of Emittance Growth}

There are four main contributions to emittance growth [38]: thermal emittance, magnetic emittance, rf emittance and space-charge emittance. 
Thermal Emittance is due to the fact that the electrons leaving the photo cathode have non-zero momenta, since in general

$$
\hbar \omega>\epsilon_{T} .
$$

Here $\hbar \omega$ is the photon energy of the light impinging the photo-cathode, and $\epsilon_{T}=$ $\epsilon_{g a p}+\epsilon_{a}$ is the threshold energy, used for semiconductors in an analogous way as the work function $\phi$ is used for the metal photo-cathodes. The energy gap between the valence and the conduction bands is given by $\epsilon_{\text {gap }}$, and $\epsilon_{a}$ is the electron affinity (the energy difference between the conduction band and the vacuum level). In the presence of an external electric field $\epsilon_{T}$ is modified to

$$
\epsilon_{T}=\epsilon_{T}^{0}-e \sqrt{\frac{e E}{4 \pi \epsilon_{0}}},
$$

where $\epsilon_{T}^{0}$ is the threshold energy of the photo-cathode without any external electric field and $E$ is the rf electric field on the photo-cathode. The effect of lowering the potential barrier in the presence of the electric field is known as the Schottky effect. The threshold energy $\epsilon_{T}^{0}$ for $\mathrm{Cs}_{2} \mathrm{Te}$ is $3.5 \mathrm{eV}$; the effective work function $\epsilon_{T}$ in the presence of an electric field of $E=30 \mathrm{MV} / \mathrm{m}$ is lowered to $3.3 \mathrm{eV}$. Flöttmann has shown [39] that for $\hbar \omega=4.7 \mathrm{eV}$ (263 nm wavelength), electrons are excited to a final state energy of $4.05 \mathrm{eV}$, and therefore the electrons leaving the photo-cathode have an average kinetic energy $\epsilon_{k}=p^{2} / 2 m$ of $0.75 \mathrm{eV}$. Assuming the electrons are emitted isotropically the maximum angle (with respect to the surface normal) is given as

$$
\theta_{\max }=\arccos \sqrt{\frac{\epsilon_{a}}{\epsilon_{k}}} .
$$

For the electron affinity $\epsilon_{a}=0.2 \mathrm{eV}, \theta_{\max }=59^{\circ}$. The corresponding transverse momentum $p_{x}$ is then

$$
p_{x}=p \sin \theta \cos \phi,
$$


where $\theta=\left[0, \theta_{\max }\right]$ and $\phi=[0,2 \pi]$ are the azimuth and meridian angles respectively. With

$$
p=m_{0} c \sqrt{\gamma^{2}-1} \approx m_{0} c \sqrt{\frac{2 \epsilon_{k}}{m_{0} c^{2}}}
$$

and

$$
\sqrt{\left\langle p_{x}^{2}\right\rangle}=\sqrt{\frac{\iint p_{x}^{2} \sin \theta d \theta d \phi}{\iint \sin \theta d \theta d \phi}}
$$

the normalized rms emittance (Eq. 3.2) is

$$
\epsilon_{n, x}^{t h}=\sigma_{x} \sqrt{\frac{2 \epsilon_{k}}{m_{0} c^{2}}} \frac{1}{\sqrt{3}} \sqrt{\frac{2+\cos ^{3} \theta_{\max }-3 \cos \theta_{\max }}{2\left(1-\cos \theta_{\max }\right)}} .
$$

For a one $\mathrm{mm}\left(\sigma_{x}=1 \mathrm{~mm}\right)$ uniform distribution and the above parameters, the thermal emittance is $\epsilon_{n, x}^{t h}=0.8 \mathrm{~mm}-\mathrm{mrad}$.

Magnetic Emittance: From Eq. 3.1 it follows explicitly that the value of the emittance depends on the canonical momentum. Thus if there exists a non-zero longitudinal magnetic field at the cathode the ensemble of particles will experience an increase of the transverse emittance because of the presence of a transverse component in the angular momentum given by

$$
p_{x}=e A_{x}=e \frac{B_{z}}{2} y
$$

where $\vec{A}$ is the vector potential defined by $\vec{B}=\vec{\nabla} \times \vec{A}$, and therefore $\vec{A}=(y \hat{x}-$ $x \hat{y}) B_{z} / 2$.

From Eqs. (3.15) and (3.2) it follows that

$$
\epsilon_{x}^{m a g}=\frac{e B_{z}}{2 m c} \sqrt{\left\langle x^{2}\right\rangle\left\langle y^{2}\right\rangle-\langle x y\rangle^{2}},
$$

and for $\langle x\rangle=\langle y\rangle=\langle x y\rangle=0$

$$
\epsilon_{x}^{m a g}=\frac{e B_{z}}{2 m c} \sigma_{x} \sigma_{y}
$$


Here we introduce the rms spread in beam position, $\sigma_{x, y}=\sqrt{\left\langle x^{2}\right\rangle-\langle x\rangle^{2}}$. The emittance-compensation solenoid peak magnetic field has a typical value on the order of $2 \mathrm{kG}$. Even though the bucking coil is used to null the magnetic field at the cathode, there is some field still present on the cathode. For $B_{z} \sim 10$ gauss, and $\sigma_{x}=\sigma_{y}=1 \mathrm{~mm}, \epsilon_{x}^{m a g}=0.07 \mathrm{~mm}-\mathrm{mrad}$ for a uniform transverse distribution; under nominal conditions of operation this is an insignificant contribution to the total emittance.

RF Emittance: At the exit of the gun the longitudinal electric field must terminate. This leads to the exit kick effect. It has been shown [38] that at the exit of the gun the radial velocity is given by

$$
\beta_{r}=\alpha k r \sin \phi_{\text {exit }}
$$

Here $\alpha$ is the normalized peak electric field

$$
\alpha=\frac{e E_{0}}{2 k m c^{2}} .
$$

with $k=2 \pi / \lambda$ the wavevector of the accelerating $\mathrm{rf}$ and $E_{0}$ the peak electric field. $r$ is the transverse distance of an electron from the gun axis and $\phi_{\text {exit }}$ is the exit phase of the gun. For $p_{x}=m c \beta_{x}=\alpha k x \sin \phi$ and from Eq. (3.2) it follows that

$$
\epsilon_{x}^{r f}=\alpha k\left\langle x^{2}\right\rangle \sqrt{\left\langle\sin ^{2} \phi\right\rangle-\langle\sin \phi\rangle^{2}}
$$

where $\phi=k z+\phi_{\text {exit }} ; z$ is the position of the electons in the longitudinal domain with respect to the center of the ensemble (obviously $\langle z\rangle=0$ ). Since $z$ is on the order of $1 \mathrm{~mm}$ and $k=0.027 \mathrm{~mm}^{-1}, k z \ll 1$ we can use the following approximation

$$
\sin \phi=\sin \left(k z+\phi_{\text {exit }}\right) \approx k z \cos \phi_{\text {exit }}+\sin \phi_{\text {exit }} .
$$

And therefore

$$
\langle\sin \phi\rangle \approx\left\langle k z \cos \phi_{\text {exit }}\right\rangle+\left\langle\sin \phi_{\text {exit }}\right\rangle=\left\langle\sin \phi_{\text {exit }}\right\rangle \equiv \sin \phi_{\text {exit }}
$$




$$
\left\langle\sin ^{2} \phi\right\rangle \approx k^{2}\left\langle z^{2}\right\rangle\left\langle\cos ^{2} \phi_{\text {exit }}\right\rangle+\left\langle\sin ^{2} \phi_{\text {exit }}\right\rangle=k^{2}\left\langle z^{2}\right\rangle \cos ^{2} \phi_{\text {exit }}+\sin ^{2} \phi_{\text {exit }}
$$

with these simplifications Eq. (3.20) becomes

$$
\epsilon_{x}^{r f} \approx \alpha k^{2} \sigma_{x}^{2} \sigma_{z}\left|\cos \phi_{\text {exit }}\right|
$$

As we can see the minimum emittance is for an exit phase $\phi=\pi / 2$. In our case $\left(E_{0}=35 \mathrm{MV} / \mathrm{m}, \lambda=23 \mathrm{~cm}\right) \alpha=1.30$, the initial phase $\phi_{0}=45^{\circ}, \phi_{\text {exit }}=$ $\phi_{0}+1 / 2 \alpha \sin \phi_{0}=76^{\circ}[41]$. For a beam size $\sigma_{z}=\sigma_{x}=1 \mathrm{~mm}$ and a uniform distribution, $\epsilon_{x}^{r f} \approx 0.3 \mathrm{~mm}-\mathrm{mrad}$.

Space-charge Emittance: An ensemble of charged particles exerts a repulsive electrical force among its constituent thus making itself to inflate. This is referred to as the space-charge effect. The linear space-charge force that acts as a defocusing lens could be corrected by employing the emittance-compensation solenoids. The non-linear space-charge forces associated with the difference in electric field between the edges of the beam and the core, cannot be corrected and cause most of the damage.

The space-charge force decreases as $1 / \gamma^{2}$ for relativistic beams because in the rest frame the size of the beam in the longitudinal domain is decreased by a factor of $\gamma$ and the proper time is decreased by a factor of $\gamma$ as well. Thus, in order to reduce the effects of space-charge emittance growth, the beam of charged particles must be rapidly accelerated to high energies.

There exists an analytical model that can predict the emittance evolution in an accelerating structure [41]:

$$
\epsilon_{x}^{s . c .} \propto \frac{1}{\alpha k \sin \phi_{0}} \frac{I}{I_{A}}
$$

where $I$ is the electron current, and $I_{A} \equiv 4 \pi \epsilon_{0} m c^{3} / e \approx 17 \mathrm{kA}$ is the Alfvén current. This model however is sensitive to the bunch shape and therefore is not 


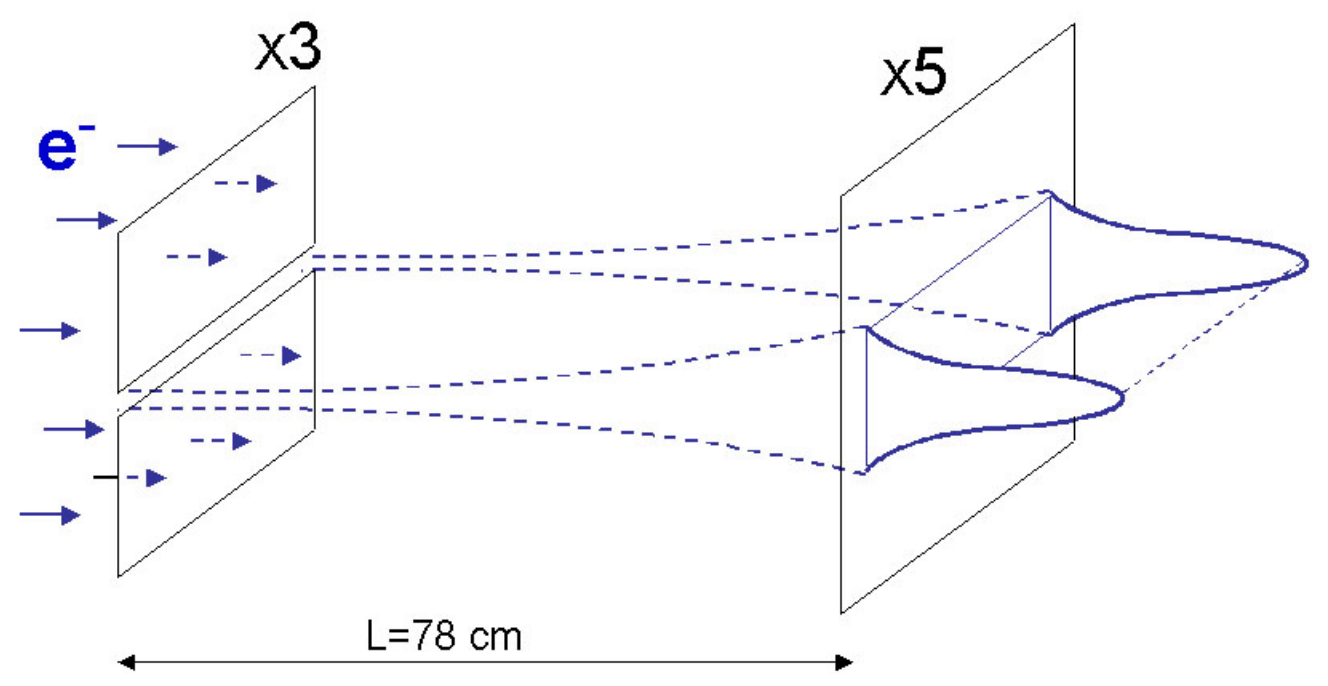

Figure 3.4: Slit method schematics. The electrons coming from the left go through the slit (X3) and hit onto the YAG flag (X5).

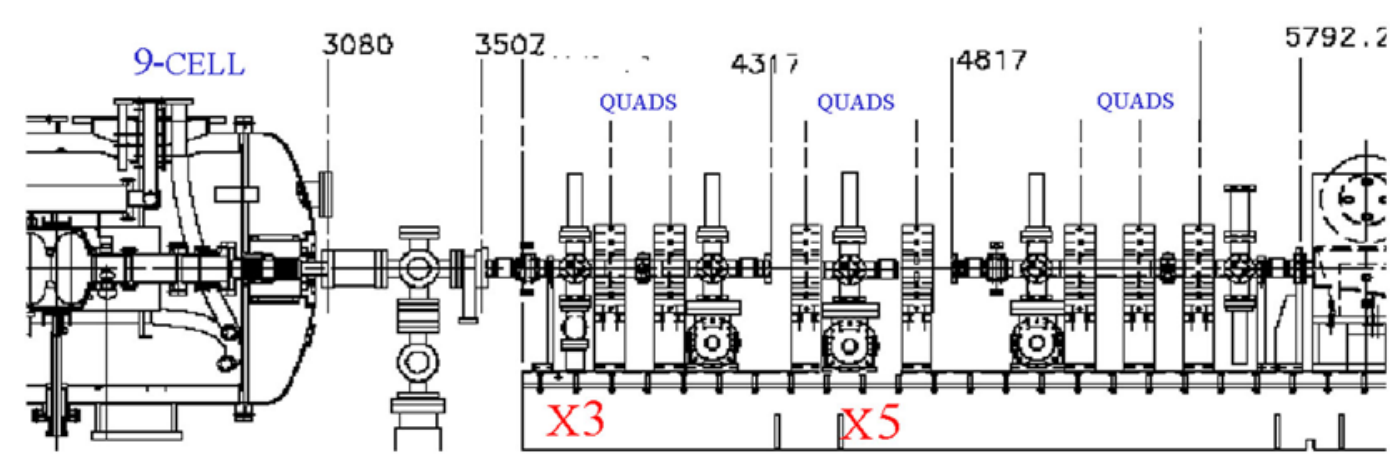

Figure 3.5: Beam line schematics (excerpt from Fig. 1.2). Emittance was measured at X3, which is the first available cross after the 9-cell cavity (left). The image of the slits at X3 was projected onto a YAG screen at X5. The numbers indicate the distance from the photocathode (in $\mathrm{mm}$ ). 
very suitable for comparative analysis of a flattop vs. Gaussian temporal distributions. A three dimensional tracking program that includes the space charge effect such as ASTRA has to be employed. ASTRA (A Space Charge Tracking Algorithm) [42] tracks particles through user defined external fields taking into account the space charge field of the particle cloud. The tracking is based on a Runge-Kutta integration of 4 th order with fixed time step. Although ASTRA is a three-dimensional code, the simplified version with a cylindrical grid was used to make the calculation fast enough for a $\mathrm{PC}$.

It is important to note that in the most general case, different parts of an ensemble of particles (electron bunch) have different values of emittance. Usually, an integrated emittance (referred to as "projected" emittance) is considered as a comparative characteristic of a beam; integration is performed in both the transverse and time domains. One can also consider measurements of the slice emittance, resolved in the time domain but integrated over the transverse dimentions (see [43], [44]). The transverse emittance distribution as well as the dependence of the integrated emittance on different parameters (such as charge and shape of the beam) is being considered in the following sections.

\subsubsection{Experimental Set-up}

The emittance was measured by using the slit technique. Slits, 50 micron wide, were made in an $\mathrm{Al}$ mask; the slit separation was $1 \mathrm{~mm}$. The mask is remotely interchangeable with an optical transition radiation (OTR) tungsten screen (which allows us to observe the transverse bunch size by means of a camera) and located at flag X3 (see Fig. 3.5). The image of the slits can be viewed on an OTR yttrium aluminum garnet (YAG) screen located at X5. The distance between the flags is 


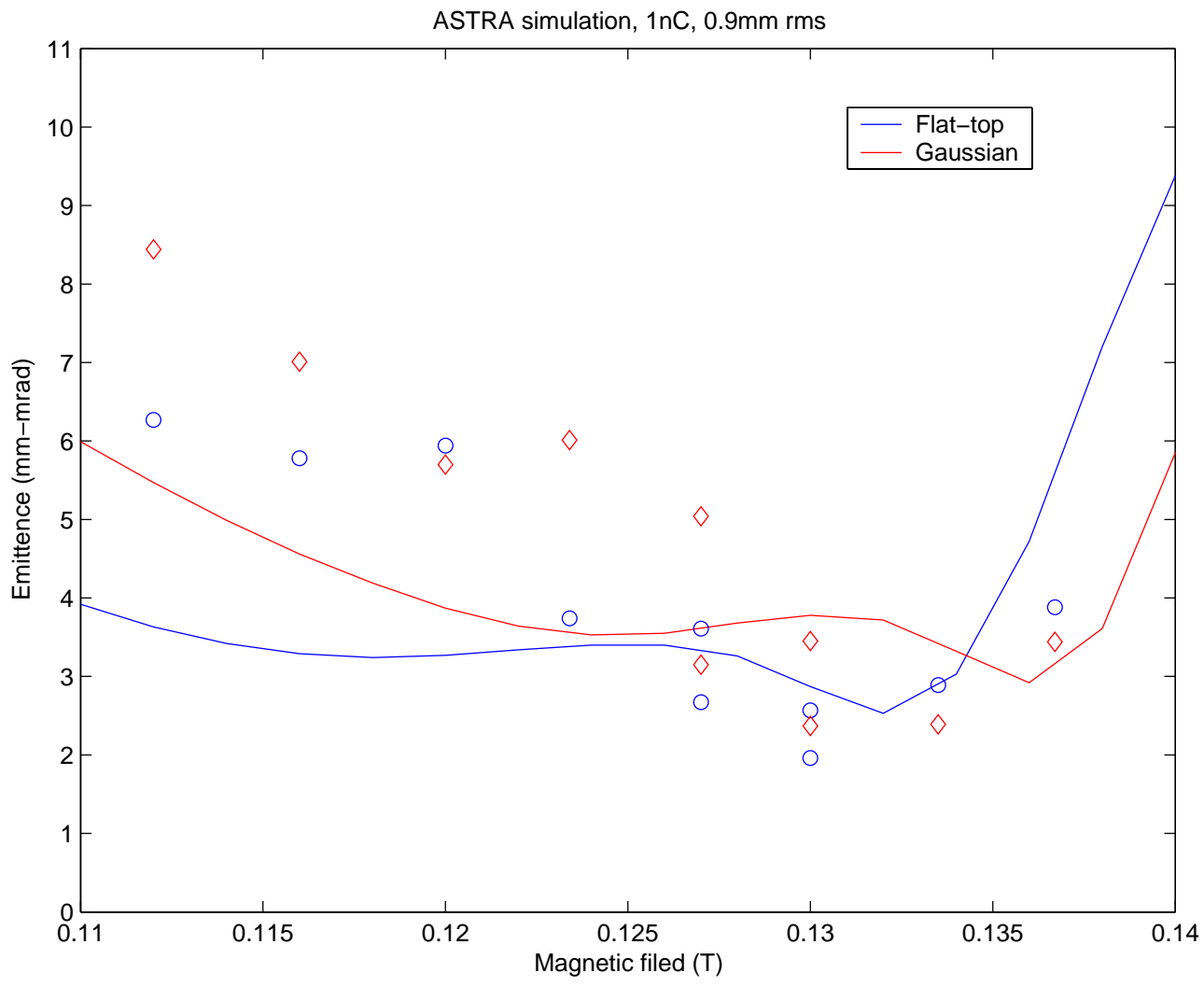

Figure 3.6: Emittance versus solenoid field: experimental points (diamonds and circles) and ASTRA simulation (solid lines). Gaussian pulse length is $3.5 \mathrm{ps}$ rms and flat-top is $6.5 \mathrm{ps} \mathrm{rms}$; the transverse size for both is $0.9 \mathrm{~mm} \mathrm{rms}$ and the charge is $1 \mathrm{nC}$. 
$78 \mathrm{~cm}$ (see Fig. 3.4). The beam divergence is calculated from the growth of the size of the slit image for a given flag distance. Beam spot size, its divergence as well as its energy determine the normalized emittence according to Eq. (3.3):

$$
\epsilon_{x}=\beta \gamma \sigma_{x} \sigma_{x^{\prime}}
$$

where $\sigma_{x}$ is the rms beam size (flag X3) and $\sigma_{x^{\prime}}=d_{x} / L$ is the rms value of the beam divergence; $d_{x}$ is the rms value of the slit image width (flag X5) and $L$ is the distance between the flags. The photoinjector linac was set to operate at its regular regime; see Table 3.1 for the list of parameters.

Table 3.1: Linac operating parameters.

\begin{tabular}{|l|l|}
\hline Laser transverse size on photocathode, rms & $0.91 \mathrm{~mm}$ \\
\hline Laser pulse length (single Gaussian), rms & $2.05 \mathrm{ps}$ \\
\hline Laser pulse length (flattop), rms & $6.57 \mathrm{ps}$ \\
\hline Charge & $1.013 \mathrm{nC}$ \\
\hline rf-gun peak field & $34.82 \mathrm{MV} / \mathrm{m}$ \\
\hline 9-cell accel. field & $12.00 \mathrm{MV} / \mathrm{m}$ \\
\hline Electron energy & $15.83 \mathrm{MeV}$ \\
\hline Separation of pulses in train & $1 \mu \mathrm{s}$ \\
\hline Number of pulses in train & 2 \\
\hline Repetition rate & $1 \mathrm{~Hz}$ \\
\hline rf frequency & $1.30 \mathrm{GHz}$ \\
\hline Laser injection phase & $45^{\circ} \pm 5^{\circ}$ \\
\hline 9-cell phase & $\sim 84^{\circ}$ \\
\hline
\end{tabular}

The emittance compensation scheme proposed by Carlsten [45] was used to minimize the projected emittance. There are three solenoids around the A0 rf- 
gun: primary, bucking and secondary. The emittance measurements were taken while varying the solenoid current in such a way that all three solenoids had the same current. This ensures that the magnetic field on the photocathode surface is zero. The current was varied from 210 to 280 Amps (which corresponds to peak fields from 0.112 to 0.137 Tesla).

\subsubsection{Results}

We have measured the emittance for both single (Gaussian) and stacked (flattop) pulses as a function of rf-gun solenoid current (see Fig. 3.6). The minimum value for a Gaussian pulse is $2.9 \mathrm{~mm}-\mathrm{mrad}(0.137 \mathrm{~T})$; and for a stacked pulse it is $2.5 \mathrm{~mm}$ mrad $(0.132 \mathrm{~T})$. The stacked pulse has lower emittance and agrees well with the ASTRA simulation. For our value of emittance $(2.5 \mathrm{~mm}-\mathrm{mrad})$, the difference of $0.4 \mathrm{~mm}$-mrad (which is only $\sim 20 \%$ ) does not seem to be very significant. However, in a properly optimized accelerator, that is in accelerator which is not only tuned properly, but also uses optimized hardware (including the proper distance between the rf-gun and the 9-cell cavity), we expect a sub $1 \mathrm{~mm}$-mrad emittance (for $1 \mathrm{nC}$ charge). For such a low emittance machine, utilization of a flattop scheme would be a great improvement. Also, for such a machine, we expect a better performance for the flattop scheme in a super large charge regime (greater than $10 \mathrm{nC}$ ).

Phase space correlation (divergence as a function of transverse distance from the beam centroid) has been analyzed for one of the settings (flattop, 0.137 T) see Fig. 3.7. As expected the divergence is smallest in the center of the beam and it grows as we move away from the center (transversely). The phase space map has been reconstructed for that setting (see Fig. 3.8) using Matlab [46]. We show the particle density distribution as a function of phase-space coordinates $\left(x, x^{\prime}\right)$. 

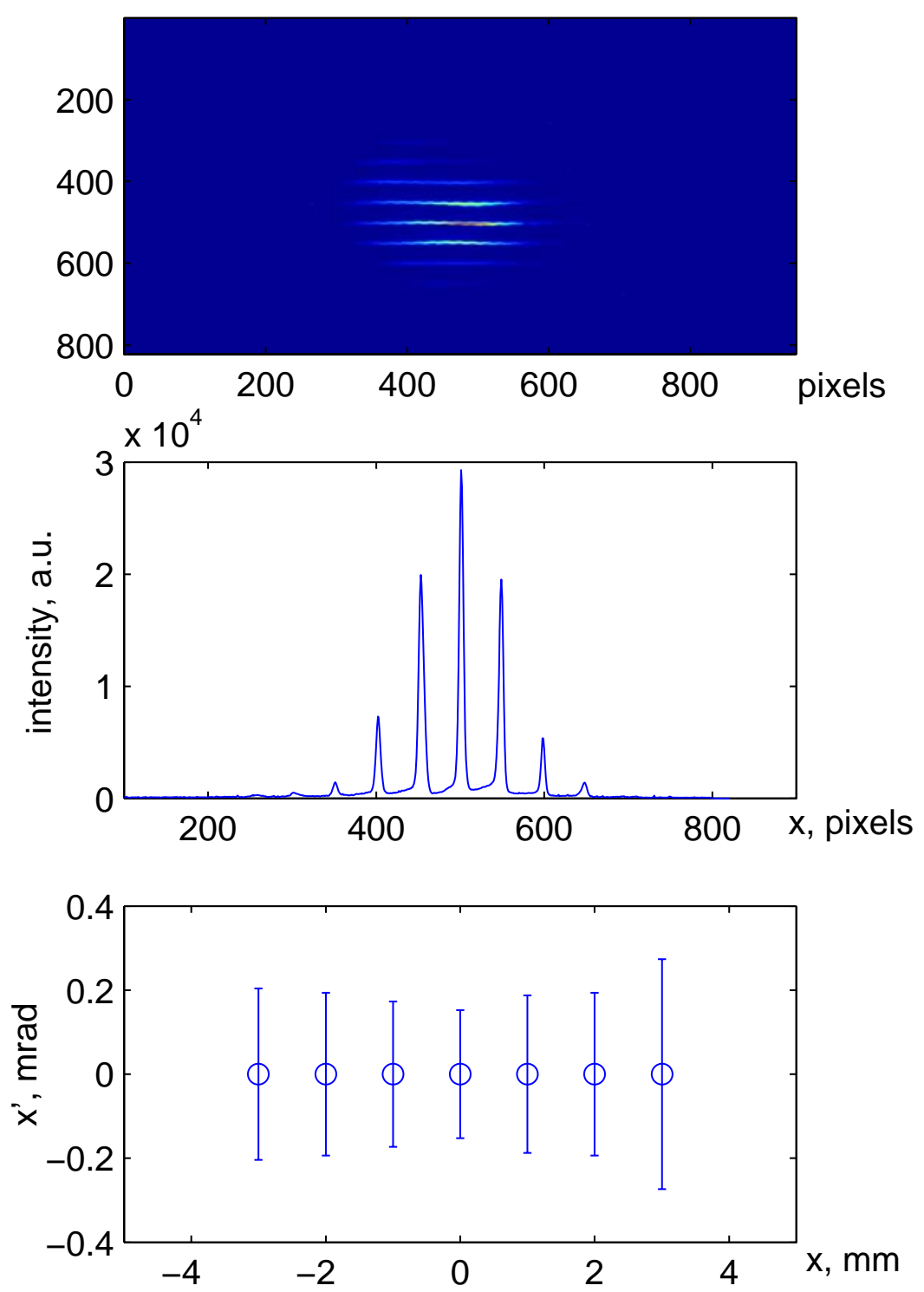

Figure 3.7: Image of slits at flag X5 (top) and its projection on the vertical axis (middle). Phase space correlation: divergence as a function of transverse distance (bottom). 


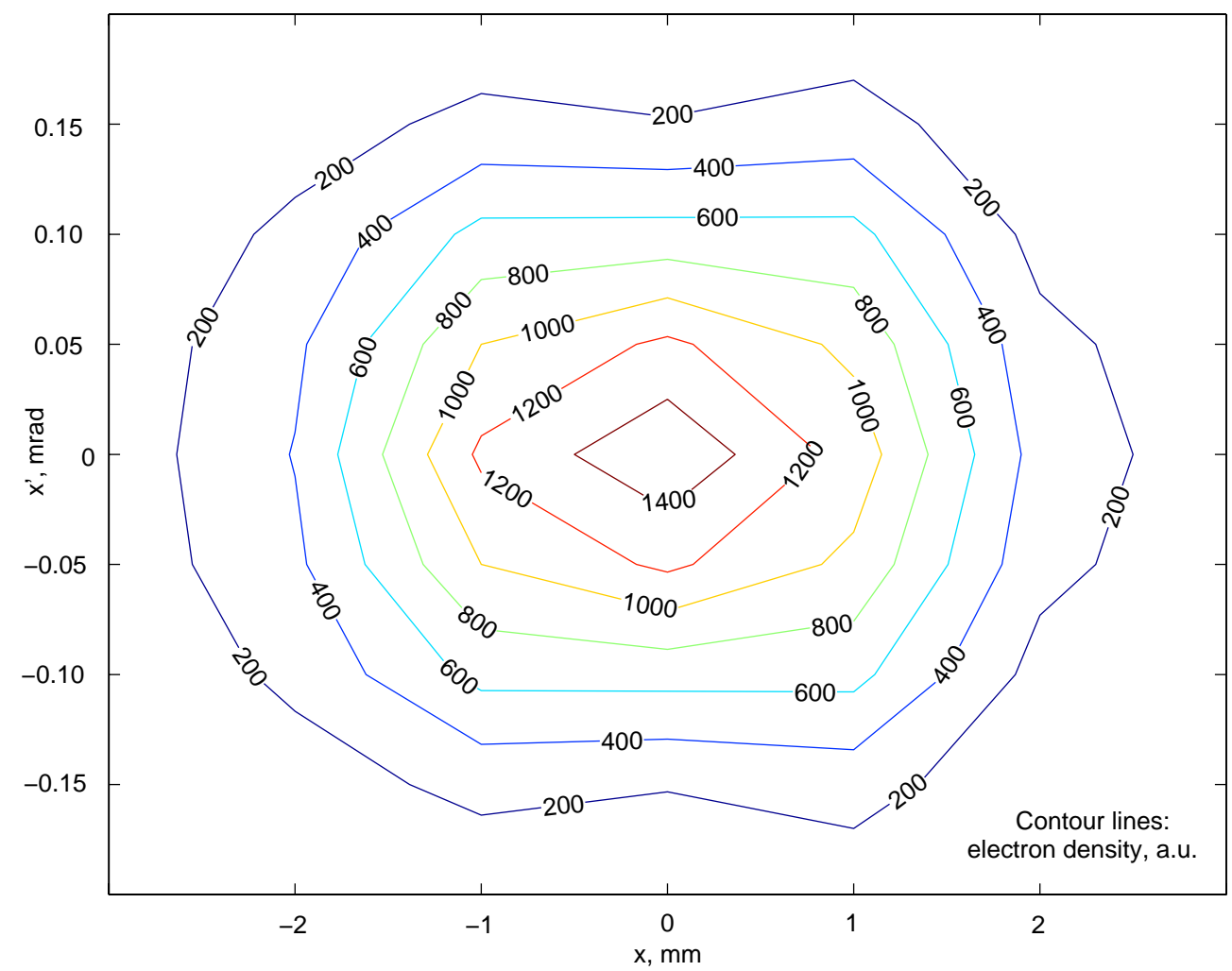

Figure 3.8: Transverse phase space map at X3 (1 nC, 20.8 ps FWHM flattop, $\left.B_{\text {sol }}=0.137 \mathrm{~T}\right)$. Electron density distribution as a function of phase-space coordinates $\left(x, x^{\prime}\right)$. This plot corresponds to the projected emittance value of 4 mm-mrad. To make the map readable, the slope of the phase space correlation (divergence of the whole electron beam, whose value is $0.4 \mathrm{mrad}$ ) was not included. 


\subsection{Pulse length as a function of charge}

Even though the set charge for the International Linear Collider was chosen to be $3.2 \mathrm{nC}$, there are experiments that require drastically different values of charge. Experiments sensitive to emittance would typically require a charge less than $1 \mathrm{nC}$. On the other hand, there are number of experiments (such as acceleration by a plasma wakefield) that require very large charge (greater than $10 \mathrm{nC}$ ). It is understood that the transverse emittance grows with charge due to the spacecharge effect. However, it is also interesting to observe how the space-charge contributes to the pulse elongation and whether such elongation depends on the original shape of the pulse.

The laser intensity was varied by means of a half-wave plate and a prism. Thus we were able to vary the charge of the electron bunch from $1 \mathrm{nC}$ to $10 \mathrm{nC}$. The aperture was set to $1.77 \mathrm{~mm}$ rms to better accommodate such a range of charges and then imaged onto the photo cathode so that diffraction effects due to the aperture were avoided. The streak camera was used to collect the light from the OTR. The dependence of pulse length on bunch charge was measured for both Gaussian and stacked pulses and is presented on Fig. 3.9. The pulse length for the Gaussian distribution has a stronger charge dependence. It agrees well with ASTRA simulation. This is due to the stronger space charge effect for the Gaussian distribution.

\subsection{Conclusion}

A long laser pulse with a quasi flattop temporal distribution was constructed by adding four single Gaussians by means of a pulsestacker. Such a laser pulse (as well as a single Gaussian) was imaged onto the photocathode and an electron 


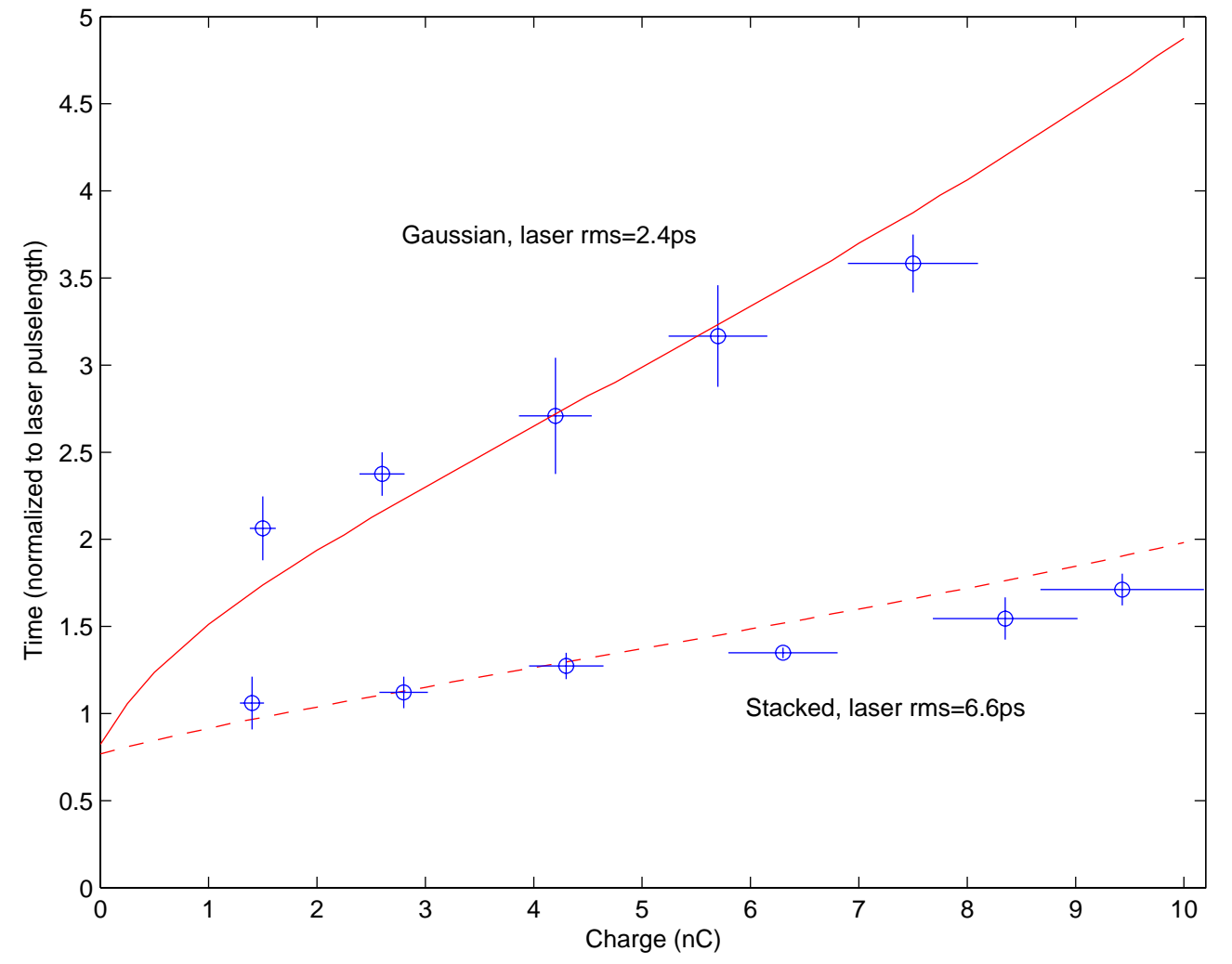

Figure 3.9: Electron bunch length normalized to the corresponding laser pulse length (2.4 ps rms for Gaussian and $6.6 \mathrm{ps}$ rms for the flattop) as a function of charge. The growth for the longer flattop pulse is significantly slower. 
pulse with corresponding temporal distribution was produced. The two temporal distributions have been compared analytically and experimentally. The minimum emittance was found for each of them by scanning the rf-gun solenoid current. The quasi flattop emittance gives $20 \%$ better value. Electron pulse elongation as a function of bunch charge was studied for both distributions. The growth for the longer flattop pulse is significantly slower.

A quasi flattop distribution in the time domain yields better results than a simple Gaussian for both the emittance measurements and space-charge effects. It is therefore preferable to use the pulse stacker for future experiments especially those requiring low emittance and/or high charge. 


\section{Chapter 4}

\section{Beam Compression}

\subsection{Introduction}

Linear accelerators designed to drive FEL-based light sources or advanced accelerator physics R\&D experiments (such as plasma wakefield accelerators) need to provide small emittance high peak current electron bunches. In order to achieve such high-brightness beams, the bunch after generation is generally manipulated both in the transverse and longitudinal phase spaces. This is achieved by emittance compensation and by bunch compression techniques. The beam dynamics associated with such beams is intricate since both the external and internal fields have to be taken into account. It is, therefore, difficult to set-up and optimize the beam manipulation process by simply measuring the bunch properties (i.e. transverse emittances, momentum spread and bunch length). Instead, it is first

necessary to make sure that the lattice is set in a proper way as obtained from numerical simulations. Direct measurement of the lattice properties is generally an easy task in the transverse phase space: one can perturb the beam trajectory with a magnetic steerer and study the perturbed orbit using beam position mon- 
itors. However, as far as the longitudinal phase space is concerned this is not as

easy. A method described in [40] used a special cavity to measure the relative time of arrival of the bunch for an initial time or energy modulation. In the present Chapter we propose a much simpler method based on generating two identical microbunches separated by a fixed time interval. We refer to this scheme as that of "two macroparticles". There are two main advantages of the two-macroparticle method: First, measuring the change of the separation between two microbunches, that is the distance between their peaks, is much easier than measuring the change of the FWHM of a single bunch; the single bunch measurement is complicated due to both the background and the shape change of the bunch. Second, the space charge effects, although present within each of the macroparticles do not affect their separation (as described in Section 4.2.3). Overall, the two-macroparticle experiment provides much cleaner measurement and it has been achieved for the first time in this work.

\subsection{Theoretical Background}

\subsubsection{Magnetic Bunch Compression}

In a magnetic-based bunch compressor an energy-dependent path length is introduced via a series of dipoles forming a chicane, see Fig. 4.1. The incoming bunch that is to be compressed is first passed through an accelerating section operated off-crest so as to introduce a time-energy correlation along the bunch (a chirp). The correlation is such that the bunch head has a lower energy than the tail. When such a bunch propagates through the magnetic chicane it gets compressed: the tail catches-up with the head of the bunch. Under a single-particle approach and using the TRANSPORT formalism [47], an electron with initial coordinates 
$\left(t_{i}, \delta_{i}\right)$ in the longitudinal phase space within the bunch is mapped downstream of the bunch compressor according to:

$$
t_{f}=t_{i}+\frac{R_{56}}{c \beta} \delta_{i},
$$

and,

$$
\delta_{f}=\delta_{i} .
$$

$R_{56}$ is the so-called first order momentum compaction for the bunch compressor, $c$ is the velocity of light. The coordinates of the electron in the longitudinal phase space are time $t_{i}$ and fractional momentum spread $\delta_{i} \equiv\left(p_{z}-\left\langle p_{z}\right\rangle\right) /\left\langle p_{z}\right\rangle$ (where $\left\langle p_{z}\right\rangle$ is the average momentum of the bunch). The $R_{56}$ notation comes from the transfer matrix formalism which deals with a six-dimensional trace space ${ }^{1}$. We should also use longitudinal distance $z$ instead of time $t$, in this case. For $z=c \beta t$ Eq. 4.1 reads $z_{f}=z_{i}+R_{56} \delta$.

A six-dimensional radius-vector in trace-space is defined as

$$
r=\left(\begin{array}{c}
x \\
x^{\prime} \\
y \\
y^{\prime} \\
z \\
\delta
\end{array}\right)
$$

where $x^{\prime} \equiv p_{x} / p_{z} ; r$ is transformed by the transfer matrix $R$

$$
r^{\prime}=R r
$$

\footnotetext{
${ }^{1}$ The trace-space is defined from the phase-space by substituting $p_{x}, p_{y}$ and $p_{z}$ with $x^{\prime}, y^{\prime}$ and $\delta$ respectively.
} 
where

$$
R=\left(\begin{array}{cccc}
R_{11} & R_{12} & \ldots & R_{16} \\
\vdots & \vdots & \ddots & \vdots \\
R_{51} & R_{52} & \ldots & R_{56} \\
R_{61} & R_{62} & \ldots & R_{66}
\end{array}\right)
$$

Clearly, $R_{56}$ corresponds to the correlation between $z$ and $\delta$.

Since $\beta \approx 1$ we can interpret $\delta$ in terms of the energy: $\delta \equiv(\mathcal{E}-\langle\mathcal{E}\rangle) /\langle\mathcal{E}\rangle$. Note that minimum bunch length is achieved $\left(t_{f}=0\right.$ under single-particle linear dynamics) provided

$$
\frac{d \delta_{i}}{d t_{i}}=\frac{c}{R_{56}}
$$

that is, the incoming chirp matches the chicane momentum compaction. Finally we should note that in the case of two macropaticles, the evolution of the macropaticle separation downstream of the bunch compressor is given by:

$$
\Delta t_{f}=\Delta t_{i}+\frac{R_{56}}{c} \frac{\Delta \mathcal{E}}{\mathcal{E}}
$$

where $\Delta \mathcal{E}$ is the macroparticle energy difference. And introducing a second order correction into Eq. 4.6 yields:

$$
\Delta t_{f}=\Delta t_{i}+\frac{R_{56}}{c} \frac{\Delta \mathcal{E}}{\mathcal{E}}+\frac{T_{566}}{c}\left(\frac{\Delta \mathcal{E}}{\mathcal{E}}\right)^{2}
$$

The second order correction tensor element $T_{566}$ should have a theoretical value of $-\frac{3}{2} R_{56}$ [49]. In such a case $R_{56}$ could be written as

$$
R_{56}=\frac{c\left(\Delta t_{f}-\Delta t_{i}\right)}{\delta(1-3 / 2 \delta)}
$$

where $\delta \equiv \Delta \mathcal{E} / \mathcal{E}$. Therefore measuring the separation and energy of each macroparticle allows us to infer the momentum compaction of the chicane. 


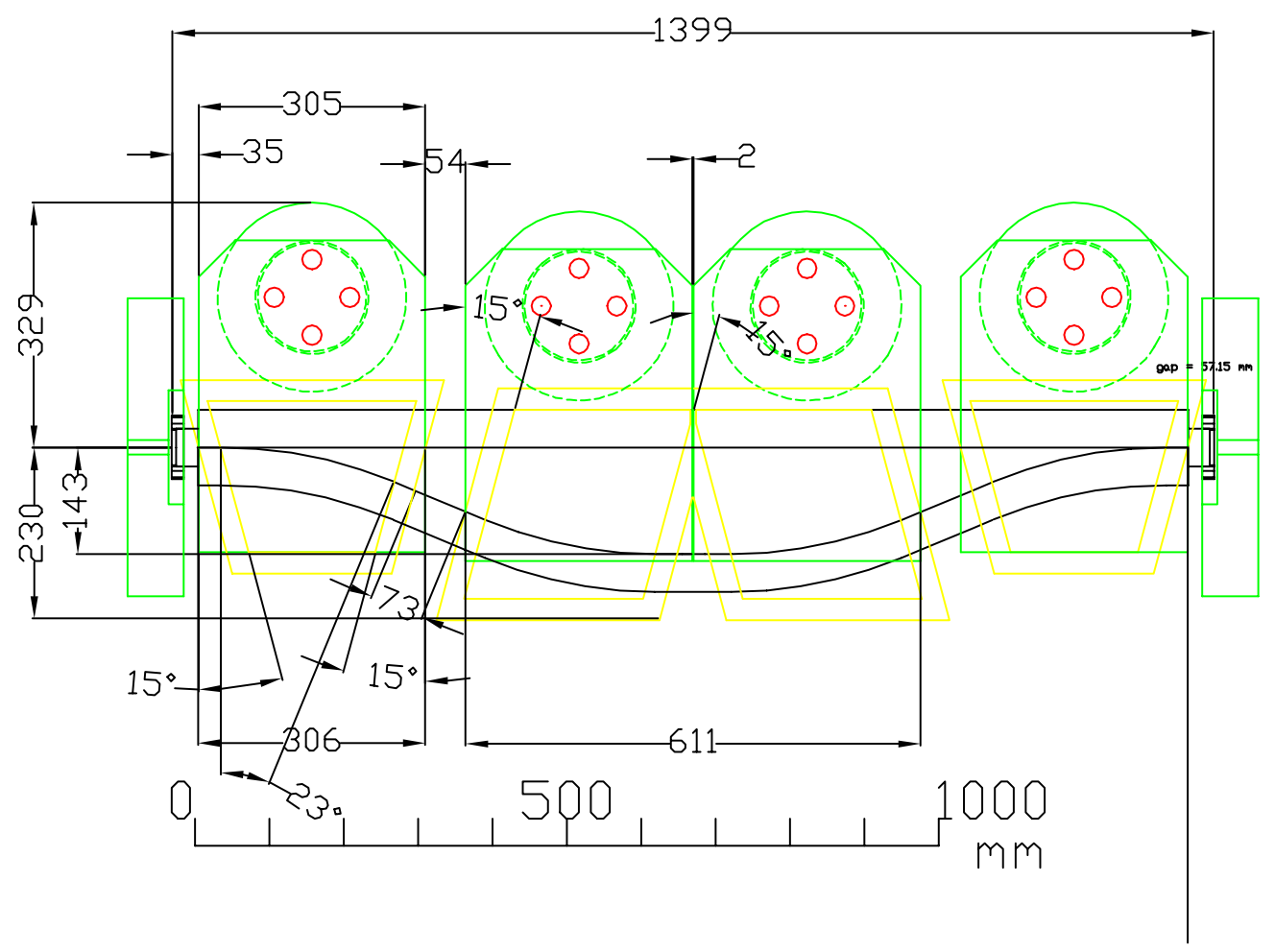

Figure 4.1: Overview of the A0 magnetic bunch compressor chicane. The plane of the drawing corresponds to the deflecting plane $y z$. The magnetic fields $\left(B_{x}\right)$ are directed into the plane of paper. 


\section{Energy Spread due to the RF-Gun}

To calculate $R_{56}$ all we need to know is the best compression phase and the ratio of the rf-gun and the 9-cell cavity energy gains. For the off-crest position, the final energy of the particles can be expressed as:

$$
\mathcal{E}(\phi)=\mathcal{E}_{0}+\mathcal{E}_{0}^{c a v} \cos \phi,
$$

where $\phi$ is the off-crest phase, $\mathcal{E}_{0}$ is the particle energy after the rf-gun, and $\mathcal{E}_{0}^{\text {cav }}$ is the particle energy gain after the 9-cell cavity for the on-crest position. Thus the maximum final energy corresponds to $\phi=0$ (on-crest position).

If we choose $\phi_{1}$ and $\phi_{2}$ to be the phase values corresponding to the best compression for the leading and trailing macroparticles respectively; then the best compression phase $\phi$ for the system of two macroparticles is approximated as $\phi=\left(\phi_{1}+\phi_{2}\right) / 2$. This is the phase when the two macroparticles collapse:

$$
\delta=\frac{\mathcal{E}_{0}^{c a v}}{\mathcal{E}_{\text {b.c. }}}\left[\cos \left(\phi-\frac{\Delta \phi}{2}\right)-\cos \left(\phi+\frac{\Delta \phi}{2}\right)\right],
$$

where $\delta \equiv \Delta \mathcal{E} / \mathcal{E}, \mathcal{E}_{\text {b.c. }}$ is the particle energy corresponding to the best compression and $\Delta \phi=\phi_{2}-\phi_{1}$ is the macroparticle phase difference. After a simple trigonometric manipulation Eq. 4.10 can be expressed as follows:

$$
\delta=\frac{2 \sin \phi \sin \left(\frac{\Delta \phi}{2}\right)}{\frac{\mathcal{E}_{0}}{\mathcal{E}_{0}^{a c v}}+\cos \phi}
$$

For $\Delta t_{f}=0$, which corresponds to the best compression, Eq. 4.6 reads:

$$
\frac{R_{56}}{c}=-\frac{\Delta t_{i}}{\delta},
$$

The macroparticle phase difference $\Delta \phi$ in terms of their initial time separation $\Delta t_{i}$ is:

$$
\Delta \phi=2 \pi \nu \Delta t_{i},
$$


where $\nu=1.3 \mathrm{GHz}$ is the rf frequency.

For small angles $\sin \left(\frac{\Delta \phi}{2}\right) \approx \frac{\Delta \phi}{2}$, so combining Eqs. 4.11, 4.12 and 4.13 we get:

$$
\begin{gathered}
\frac{R_{56}}{c}=-\frac{\frac{\mathcal{E}_{0}}{\mathcal{E}_{0}^{a c}}+\cos \phi}{2 \pi \nu \sin \phi}, \\
-R_{56}=\frac{c}{2 \pi \nu} \frac{\frac{\mathcal{E}_{0}}{\mathcal{E}_{0}^{a d v}}+\cos \phi}{\sin \phi}=3.67 \frac{\frac{\mathcal{E}_{0}}{\mathcal{E}_{0}^{\text {cav }}}+\cos \phi}{\sin \phi} \quad(\mathrm{cm})
\end{gathered}
$$

For $\mathcal{E}_{0}=3.3 \mathrm{MeV}, \mathcal{E}_{0}^{\text {cav }}=12 \mathrm{MeV}$ and if $\phi$ is equal to $35^{\circ}$, then $R_{56}$ has the value of $-7.0 \mathrm{~cm}$.

In the derivation above, we did not take into account the energy spread due to the rf-gun. Such a spread $\delta_{r f}$ results in a negative contribution to the spread caused by the 9-cell cavity being run "off-crest"

$$
\delta^{\prime}=\delta-\delta_{r f}
$$

We obtained an empirical expression for $\delta_{r f}$ from a simulation

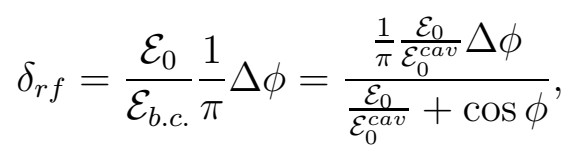

where $\Delta \phi$ is in radians. Combining Eqs. 4.11, 4.16 and 4.17, the total energy spread is given

$$
\delta^{\prime}=\frac{2 \sin \phi \sin \left(\frac{\Delta \phi}{2}\right)-\frac{1}{\pi} \frac{\mathcal{E}_{0}}{\mathcal{E}_{0}^{\text {cou }}} \Delta \phi}{\frac{\mathcal{E}_{0}}{\mathcal{E}_{0}^{\text {cav }}}+\cos \phi} \approx \frac{\left(\sin \phi-\frac{1}{\pi}\right) \frac{\mathcal{E}_{0}}{\mathcal{E}_{0}^{\text {cav }}} \Delta \phi}{\frac{\mathcal{E}_{0}}{\mathcal{E}_{0}^{\text {cav }}}+\cos \phi}
$$

Therefore Eq. 4.15 in this case will be modified as follows:

$$
-R_{56}=3.67 \frac{\frac{\mathcal{E}_{0}}{\mathcal{E}_{0}^{\text {ov }}}+\cos \phi}{\sin \phi-\frac{1}{\pi} \frac{\mathcal{E}_{0}}{\mathcal{E}_{0}^{\text {cav }}}}=3.67 \frac{\mathcal{E}_{0}+\mathcal{E}_{0}^{\text {cav }} \cos \phi}{\mathcal{E}_{0}^{c a v} \sin \phi-\frac{1}{\pi} \mathcal{E}_{0}} \quad(\mathrm{~cm})
$$

For our case of $\phi=35^{\circ}$, we find $R_{56}=-8.3 \mathrm{~cm}$.

Comparing the $R_{56}$ values for both corrected and uncorrected cases ( $\delta$ versus $\left.\delta^{\prime}\right)$, we see that the effect of the energy spread due to the rf-gun $\left(\delta_{r f}\right)$ is equivalent to shifting the 9-cell cavity phase $\phi$ by $6^{\circ}$. This agrees well with the observed discrepancy between the "on-crest" (highest energy) and the least energy spread phases. 


\subsubsection{Velocity Bunching in the RF-Gun and 9-cell Cavity}

An electron in an rf standing wave accelerating structure experiences the longitudinal electric field:

$$
E_{z}(z, t)=E_{0} \cos (k z) \sin \left(\omega t+\psi_{0}\right)
$$

where $E_{0}$ is the peak accelerating field and $\psi_{0}$ is the injection phase.

Let $\psi(z, t)=\omega t-k z+\psi_{0}$ be the phase of the electrons with respect to the rf wave. The evolution of $\psi(z, t)$ can be expressed as a function of $z$ :

$$
\frac{d \psi}{d z}=\omega \frac{d t}{d z}-k=k\left(\frac{\gamma}{\sqrt{\gamma^{2}-1}}-1\right)
$$

where $\gamma \equiv 1 / \sqrt{1-\beta^{2}}=\mathcal{E} / m c^{2}, \mathcal{E}$ and $m$ are the energy and mass of an electron respectively.

The energy gain gradient can be written as:

$$
\frac{d \gamma}{d z}=\alpha k(\sin (\psi+2 k z)+\sin (\psi))
$$

and $\alpha=e E_{0} / 2 k m c^{2}$.

The system of coupled differential equations 4.21 and 4.22 describes the longitudinal motion of an electron in the rf structure. There is no exact algebraic solution to such a system ${ }^{2}$; rather, numeric simulations such as ASTRA are successfully used to track particles through the rf gun and 9-cell cavity.

\subsubsection{Estimate of Space Charge Effects on the Separation between Two Macroparticles}

\section{(a) Introduction}

Here, we estimate the effect of space charge on the separation between the two

\footnotetext{
${ }^{2}$ The system can be solved if we neglect the backward wave term in Eq. 4.22.
} 
particles. We find that the effect is minimal but present the calculation because of it intrinsic interest.

The linac configuration is shown in Fig. 4.2, where $z_{0}$ corresponds to the rf-gun position, $z_{1}, z_{2}$ the beginning and the end of the 9-cell cavity, and $z_{3}$ the end of the linac.

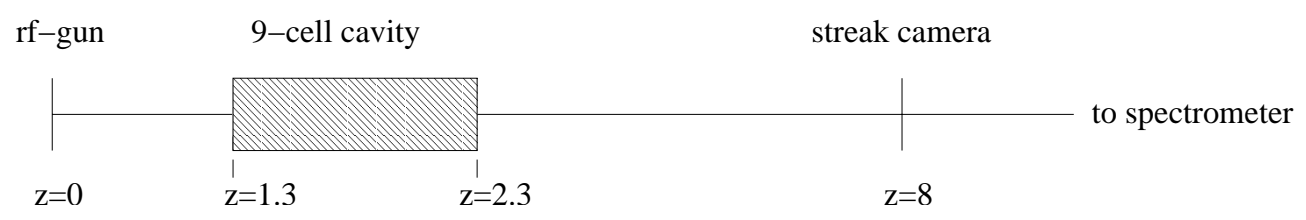

Figure 4.2: Simplified block diagram of the A0 beamline.

Consider two macroparticles $2 \mathrm{nC}$ each, separated by $5 \mathrm{~mm}(17 \mathrm{ps})$. The spacecharge effect introduces a change in momentum $\Delta P$ (two particles are pushed away from each other). There are three sources of $\Delta P$ :

- rf-Gun (kick)

- 9-cell Cavity (kick)

- Coulomb force effect over the time of traversal in the linac

The momentum change $\Delta P$ translates into a separation increase $\Delta l$ over the distance $L$ (traveled in the laboratory frame)

$$
\Delta l=\frac{L}{\gamma^{2}} \frac{\Delta P}{P},
$$

where normalized notation is used for momentum and its change, $P=\gamma \beta$. In the most general case the differential length change can be written as

$$
d \Delta l=\frac{d z}{\gamma^{2}(z)} \frac{\Delta P(z)}{P(z)}
$$

The following reasonable assumptions are applicable: 
$\Delta P$ is small ( $c \Delta P$ is much smaller than the electron rest energy)

The Coulomb force is constant (this assumes both, no loss of electrons and negligible change of the distance)

$\beta \approx 1$ (for $\gamma_{1}=8, \beta=0.992$ ), thus $\gamma \approx P$. Then Eqs. 4.23 and 4.24 become:

$$
\begin{array}{r}
\Delta l=\frac{L}{\gamma^{3}} \Delta P, \\
d \Delta l=d z \frac{\Delta P(z)}{\gamma^{3}(z)}
\end{array}
$$

Next we consider the sources of $\Delta P$ in detail.

(b) RF-Gun

From Eq. (40) of reference [41], the Coulomb force an electron is experiencing is

$$
F=\frac{1}{\gamma^{2}} f(\gamma)
$$

where $f(\gamma)$ is a slowly varying function, it can be approximated as $f(1)$. In the case of two macroparticles $f(1)=e E_{z}$, where $E_{z}=k q / r^{2}$, for the electrons at rest in the laboratory frame.

From eq. (43) of reference [41], the normalized change of momentum is expressed as

$$
\Delta P=\frac{1}{m c} \int F d t=\frac{1}{m c^{2}} \int \frac{1}{\gamma^{2} \beta} f(\gamma) d z,
$$

and from eq. (44) of reference [41]

$$
\Delta P=\frac{1}{e E_{0} \sin \phi_{0}} f(1) \int_{1}^{\gamma_{f}} \frac{d \gamma}{\gamma^{2} \beta},
$$

where $E_{0}$ and $\phi_{0}$ are the rf-gun gradient and injection phase respectively.

The above integration can be done analytically,

$$
I_{1}=\int_{1}^{\gamma_{f}} \frac{d \gamma}{\gamma^{2} \beta}=\int_{1}^{\gamma_{f}} \frac{1}{\sqrt{1-\frac{1}{\gamma^{2}}}} \frac{1}{\gamma^{2}} d \gamma=\left[\frac{\pi}{2}-\sin ^{-1}\left(\frac{1}{\gamma_{f}}\right)\right],
$$


Thus, we finally obtain for the momentum change due to the rf-gun

$$
\Delta P_{1}=\frac{1}{E_{0} \sin \phi_{0}} E_{z} I_{1}
$$

For $E_{z}=k q / r^{2}=0.8 \mathrm{MV} / \mathrm{m}, E_{0}=34 \mathrm{MV} / \mathrm{m}, \phi_{0}=45^{\circ}, I_{1}=1.45$ and therefore $\Delta P_{1}=0.048$.

In order to calculate the separation change $\Delta l_{1}$ caused by $\Delta P_{1}$ using Eq. 4.25 we need to consider three regions. First, between the gun and the 9-cell Cavity (from $z_{0}$ to $z_{1}$ ), where $\gamma_{1}=8$. Second, after the 9-cell Cavity (from $z_{2}$ to $z_{3}$ ), where $\gamma_{2}=32$, and, lastly, inside the 9-cell (from $z_{1}$ to $z_{2}$ ), where $\gamma(z)$ is changing from 8 to 32 , thus:

$$
\Delta l_{1}=\Delta P_{1} \int \frac{d z}{\gamma^{3}}=\Delta P_{1} \frac{\left(z_{1}-z_{0}\right)}{\gamma_{1}^{3}}+\Delta P_{1} \frac{\left(z_{3}-z_{2}\right)}{\gamma_{2}^{3}}+\Delta P_{1} \int_{z_{1}}^{z_{2}} \frac{d z}{\gamma(z)^{3}}
$$

Here $\gamma(z)=\gamma_{1}+E_{0}^{c a v}\left(z-z_{1}\right)$, where $E_{0}^{c a v}=12 \mathrm{MV} / \mathrm{m}$ is the 9-cell cavity gradient. The resulting separation increase $\Delta l_{1}=0.144 \mathrm{~mm}$.

(c) 9-cell Cavity

The case of the 9-cell cavity is a little harder to investigate because of it finite length. From Eq. 4.29 it follows that

$$
\Delta P_{2}(z)=\frac{1}{e E_{0}^{c a v} \sin \psi_{0}} f(1) \int_{\gamma_{1}}^{\gamma_{z}} \frac{d \gamma}{\gamma^{2} \beta}
$$

where $f(1)=e E_{z}, E_{z}=k q / r^{2}=0.8 \mathrm{MV} / \mathrm{m}$ for $2 \mathrm{nC}$ separated by $5 \mathrm{~mm}$. Further $E_{0}^{c a v}=12 \mathrm{MV} / \mathrm{m}, \psi_{0}=0^{\circ}, \beta \approx 1, \gamma_{z}=\gamma(z)$. It follows that

$$
\Delta P_{2}(z)=\frac{E_{z}}{E_{0}^{c a v}} \int_{\gamma_{1}}^{\gamma_{z}} \frac{d \gamma}{\gamma^{2}}=\frac{E_{z}}{E_{0}^{\text {cav }}}\left(\frac{1}{\gamma_{1}}-\frac{1}{\gamma(z)}\right)
$$

and for the separation change $\Delta l_{2}$

$$
\Delta l_{2}=\int d z \frac{\Delta P_{2}(z)}{\gamma^{3}(z)}
$$


Combining (12) and (13) will result in

$$
\begin{aligned}
\Delta l_{2} & =\frac{E_{z}}{E_{0}^{\text {cav }}} \int_{z_{1}}^{z_{3}}\left(\frac{1}{\gamma_{1}}-\frac{1}{\gamma(z)}\right) \frac{d z}{\gamma^{3}(z)} \\
& =\frac{E_{z}}{E_{0}^{\text {cav }}}\left[\int_{z_{1}}^{z_{2}} \frac{d z}{\gamma_{1} \gamma^{3}(z)}+\int_{z_{2}}^{z_{3}} \frac{d z}{\gamma_{1} \gamma_{2}^{3}}-\int_{z_{1}}^{z_{2}} \frac{d z}{\gamma^{4}(z)}-\int_{z_{2}}^{z_{3}} \frac{d z}{\gamma_{2}^{4}}\right],
\end{aligned}
$$

where $\gamma_{1}=8, \gamma_{2}=32, \gamma(z)=\gamma_{1}+E_{0}^{c a v}\left(z-z_{1}\right), E_{0}^{c a v}=12 \mathrm{MV} / \mathrm{m}$. Thus the separation increase is $\Delta l_{2}=16$ microns.

(d) Coulomb force effect during the traversal of the linac

For a constant force $F$, Eq. 4.28 can be written as

$$
\Delta P(t)=\frac{1}{m c} \int F d t=\frac{1}{m c} F \Delta t
$$

Expressing this in z-coordinates and making use of Eq. 4.27

$$
\Delta P(z)=\frac{1}{m c^{2}} F z=\frac{e E_{z}}{m c^{2}} \frac{z}{\gamma^{2}(z)},
$$

Introducing Eq. 4.38 into Eq. 4.35 we see that

$$
\Delta l_{3}=\frac{e E_{z}}{m c^{2}} \int \frac{z d z}{\gamma^{5}(z)}=\frac{e E_{z}}{m c^{2}}\left[\int_{z_{0}}^{z_{1}} \frac{z d z}{\gamma_{1}^{5}}+\int_{z_{1}}^{z_{2}} \frac{z d z}{\gamma^{5}(z)}+\int_{z_{2}}^{z_{3}} \frac{z d z}{\gamma_{2}^{5}}\right],
$$

where $\gamma_{1}=8$ and $\gamma_{2}=32 ; \gamma(z)=\gamma_{1}+E_{0}^{\text {cav }}\left(z-z_{1}\right)$, and $E_{0}^{\text {cav }}=12 \mathrm{MV} / \mathrm{m}$. The resulting separation increase $\Delta l_{3}=18$ microns.

(e) Results

Adding all parts yields

$$
\Delta l_{\text {total }}=\Delta l_{1}+\Delta l_{2}+\Delta l_{3}=0.178 \mathrm{~mm} .
$$

This investigation showed the biggest contribution to the increase of macroparticle separation due to space charge effect happens before the 9-cell cavity. This is to be expected since it corresponds to the lowest energy. Overall, the effect is about $4 \%$ (less than $1 \mathrm{ps}$ ), which falls within the experemental error-bars. Note that in the above investigation, we considered the worst case configuration. For the 
rf-gun and the 9-cell Cavity compression study, which requires higher resolution, we used $1.5 \mathrm{nC}$ per macroparticle and $20 \mathrm{ps}$ separation and in this case the effect is only $2 \%$.

\subsubsection{Numerical Simulations}

The numerical simulation program that we used for the velocity bunching (9cell and rf-gun compression) experiments is ASTRA (A Space Charge Tracking Algorithm) developed at DESY [42].

We have also developed a Matlab [46] program to have $R_{56}$ estimated directly from the measured B-field ${ }^{3}$. The two-dimensional B-field map was measured on the symmetry plane of the chicane $B_{x}(x=0, y, z)$, where $x, y$, and $z$ are the accelerator coordinate system (the dipoles are oriented to provide a $B_{x}$-field). The measured fields were extrapolated using an exponential function fit to reconstruct the fringe fields. Using these corrected fields the equation of motion in the $y$-plane and for $x=0$

$$
\gamma m c \frac{d^{2} y}{d z^{2}}=-e \beta_{z} B_{x}(z, y)
$$

was numerically solved. A fourth order Runge-Kutta method implemented in MATLAB was used to integrate this ordinary differential equation. An example of a calculated trajectory along the bunch compressor chicane is shown in Fig. 4.3. From the trajectory, $y(z)$, one can compute the path length variation introduced by the bunch compressor as:

$$
\delta L=\int \sqrt{d y^{2}(z)+d z^{2}}-d z
$$

which can be evaluated by a simple summation.

\footnotetext{
${ }^{3}$ B-field map measurement were provided by the Technical Division
} 

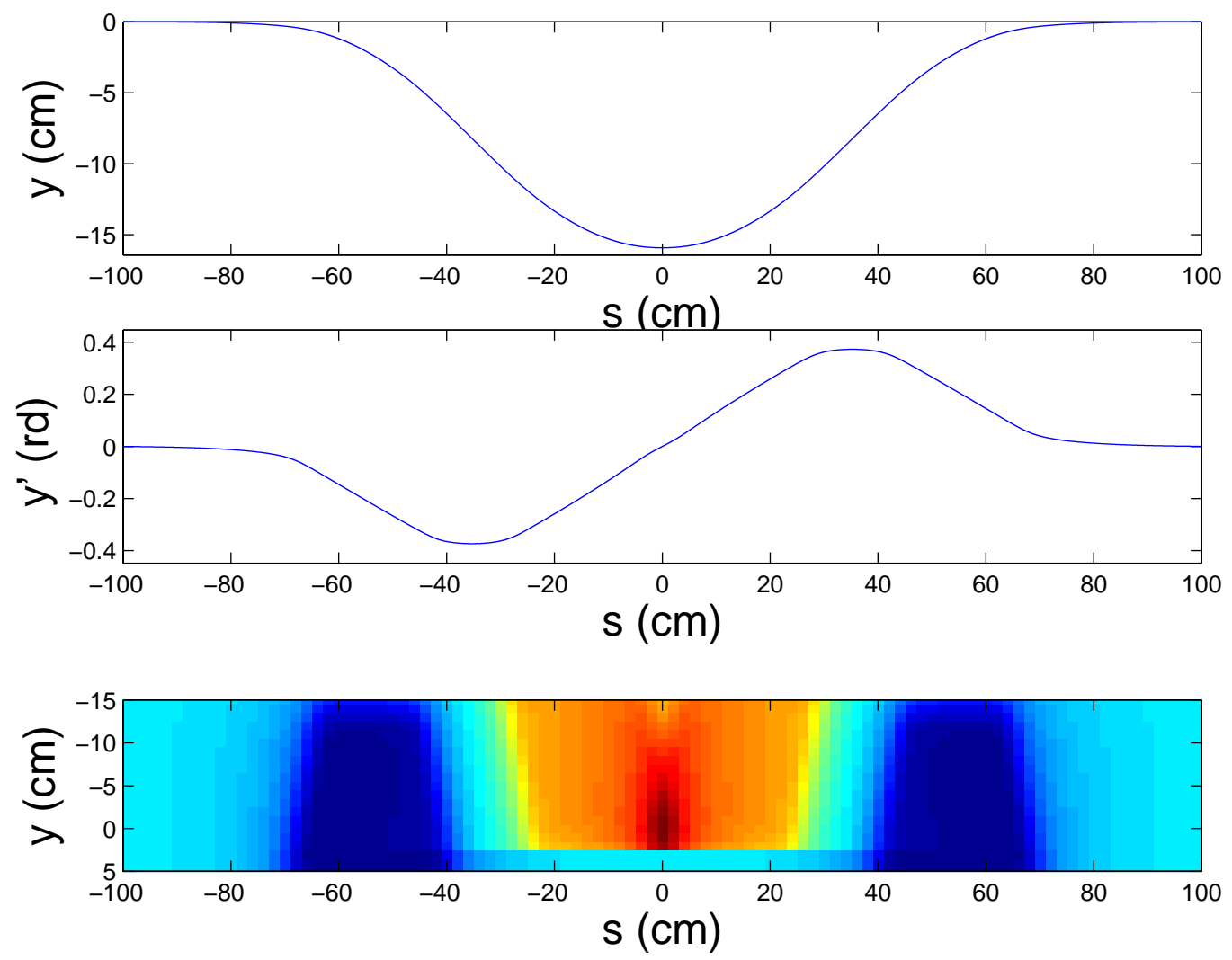

Figure 4.3: Example of integration of equation of motion: vertical trajectory offset $y$ (top) and vertical deflection (middle) versus longitudinal coordinate. Bottom 2D field false color map of the chicane $B_{x}(y, s)$-field. 
To estimate $R_{56}$, the incoming particle Lorentz factor $\gamma$ was varied around its nominal value to yield a relative variation of $\pm 1 \%$, and the corresponding path length variation, $\delta L$, was calculated for each value of $\gamma$. The value of $R_{56}$ can therefore be estimated as

$$
R_{56}=\frac{d \delta L}{d \delta},
$$

where $\delta \equiv \delta \gamma / \gamma$ is the fractional change of $\gamma$ with respect to its nominal value. The calculated values (as well as their measured counterparts) are given in Table 4.1.

\subsection{Experimental Set-up}

The beam is compressed due to velocity bunching in the rf gun and 9-cell cavity and in transversing the chicane.

The pulse stacker discussed in section 3.2 was used for this study. To create two-macroparticle configuration, the pulse stacker was operated in the single-stage regime, i.e. making one pair of copies. The two Gaussians were separated by the distance greater than their FWHM thus making the laser beam a double-pulse rather than a flattop. When such a double-pulse impinges on the photocathode it creates two electron bunches with a time separation much smaller than the rf period (769 ps). Hence both macroparticles fall into the same rf bucket and can be treated as a single bunch. The macroparticles are refered to as leading and trailing. The latter is the delayed one.

At the end of the beamline, the electron beam can be horizontally bent in a dispersive section $(B=0.14 \mathrm{~T})$, to measure the beam energy distribution using a fluorescent screen located downstream at a (horizontal) dispersion of $\left|\eta_{x}\right|=$ $317 \mathrm{~mm}$, where dispersion is defined from the transverse deflection $x=\eta \cdot \frac{\Delta P}{P}$. 
The bunch length measurement is performed with the streak camera that streaks optical transition radiation (OTR) pulses emitted as the bunch strikes an Al-coated mirror.

An alternative frequency-domain bunch length diagnostics based on MartinPuplett interferometry of coherent transition radiation is also available. The bunch longitudinal density is monitored by detecting the coherent transition radiation (CTR, $\lambda \sim 1 \mathrm{~mm}$ ) emitted in the backward direction as the electron bunch crosses the Al-coated mirror. The power density radiated by a bunch of $N$ electrons is

$$
\left[\frac{d P}{d \omega d \Omega}\right]_{N}=\left[\frac{d P}{d \omega d \Omega}\right]_{1} \times N\left(1+(N-1)|\mathcal{F}|^{2}\right)
$$

where $\left[\frac{d P}{d \omega d \Omega}\right]_{1}$ is the single electron power density and $\mathcal{F} \equiv \int_{-\infty}^{+\infty} d t S(t) \exp (-i \omega t)$ is the Fourier transform of the bunch temporal distribution $S(t)$. Since the Fourier transform of a bunch with characteristic length $\sigma_{s}$ extends to frequency $\omega \sim c / \sigma_{s}$, detecting the CTR in this frequency range provides indirect information on the bunch length. For measurements reported hereafter, only the total power of the CTR emission was detected using a pyroelectric detector, rather than measuring the cross-correlation. By maximizing the CTR emission, we could obtain the setting of minimum bunch length. The CTR detector proved to be a very good method for finding the rf phase corresponding to the best compression. Its precision is much higher than that of the streak camera and yields results better than one degree in the rf phase. However outside the region of the best compression the CTR intensity drops quickly to an undetectable level thus making it necessary to use the streak camera. 


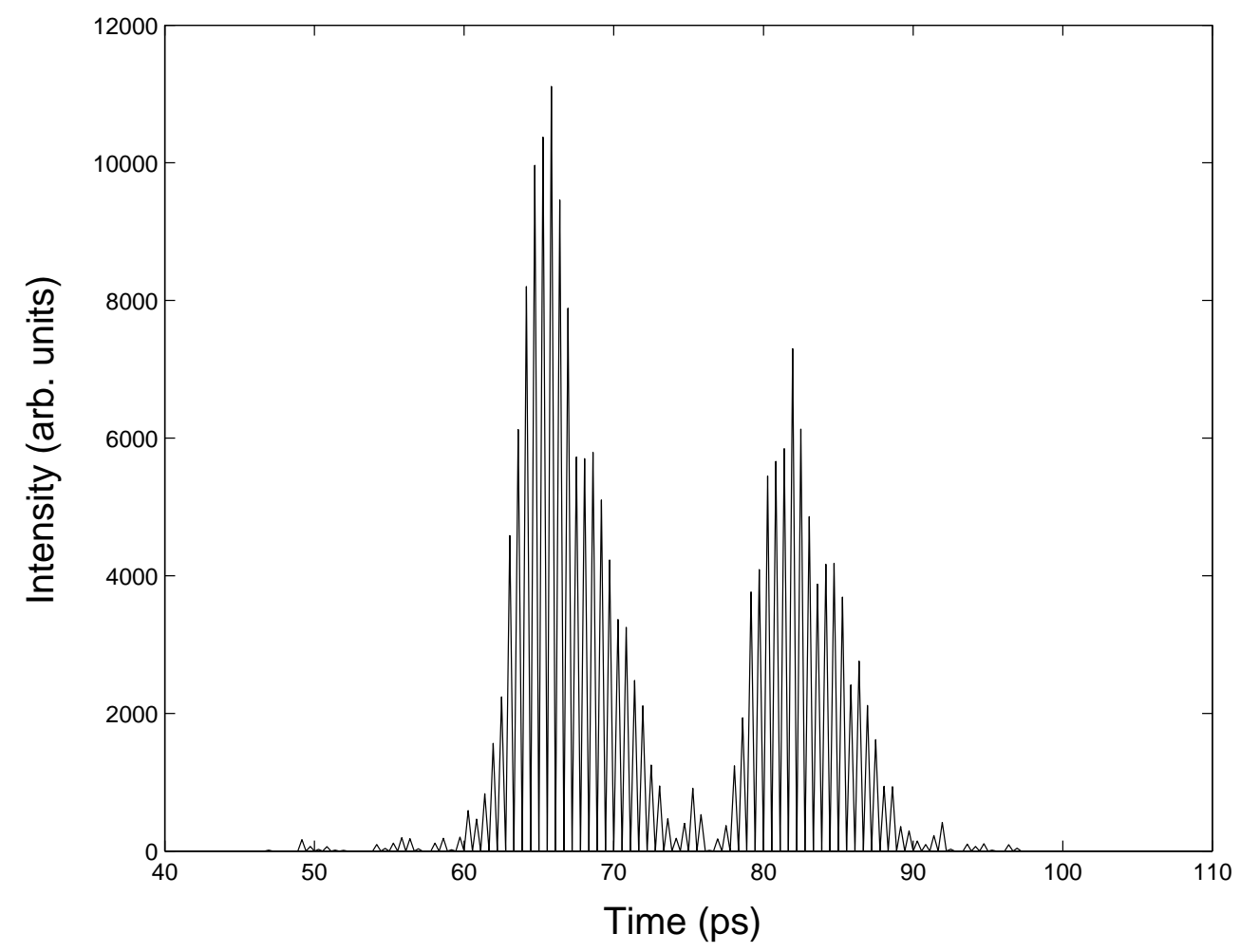

Figure 4.4: Streak camera profile of the laser set-up so as to create the twomacroparticle configuration. 


\subsubsection{Magnetic Bunch Compression Measurement}

The 9-cell cavity phase was first adjusted to the "on-crest" setting, that is, to the phase corresponding to the maximum energy gain (henceforth referred to as $\phi=0^{\circ}$. The beam energy was measured to be $15.3 \mathrm{MeV}$ ), the charge was set to be $\sim 1.6 \mathrm{nC}$ per macroparticle and the double-beam set-up was set to $17.8 \mathrm{ps}$ optical path difference, see Fig. 4.4.

The 9-cell cavity phase was then changed to approximately $-35^{\circ}$ to introduce the required chirp for compressing the bunch in the magnetic chicane. The dipoles of the magnetic chicane were excited to their nominal value ( $I=1.8 \mathrm{~A}$, corresponding to a bending angle of $22.5^{\circ}$ ). The CTR signal was used to find the phase corresponding to the best compression for each macroparticle (Fig. 4.5); the results are: off crest $-39^{\circ}$ and $-31^{\circ}$ for the leading and trailing beam (macroparticle) respectively. The next step was to go back to the un-compressed scenario (magnetic chicane dipoles un-excited and degaussed) and measure the energy spread and time difference between the two macroparticles. The energy measurements are given in Fig. 4.6 and give the macroparticle energy difference $\Delta \mathcal{E}=0.84 \mathrm{MeV}$ for the average energy $\mathcal{E}=12.9 \mathrm{MeV}(\Delta \mathcal{E} / \mathcal{E}=6.5 \%)$. The time separation between the two macroparticles was measured with the streak camera: $\Delta t_{i}=17.8$ ps. From Eq. 4.6 this yields the value $R_{56}=8.2 \mathrm{~cm}$. If the higher order correction $T_{566}$ is to be used (Eq. 4.7), the $R_{56}$ has a value of $9.1 \mathrm{~cm}$.

We can cross-check our direct results exploiting the corresponding phase measurements. The time between the macroparticles of 17.8 ps correlates well with their phase difference of $8^{\circ}$ (Fig. 4.7) which corresponds to 17.1 ps. The energy drop from the "on-crest" position $(15.3 \mathrm{MeV})$ to the "best-compression" position $(12.9 \mathrm{MeV})$ then corresponds to a phase angle of $36.9^{\circ}$ off-crest (see Eq. 4.9), which corresponds well to $35^{\circ}$ as measured. The measured phase values for each particle 


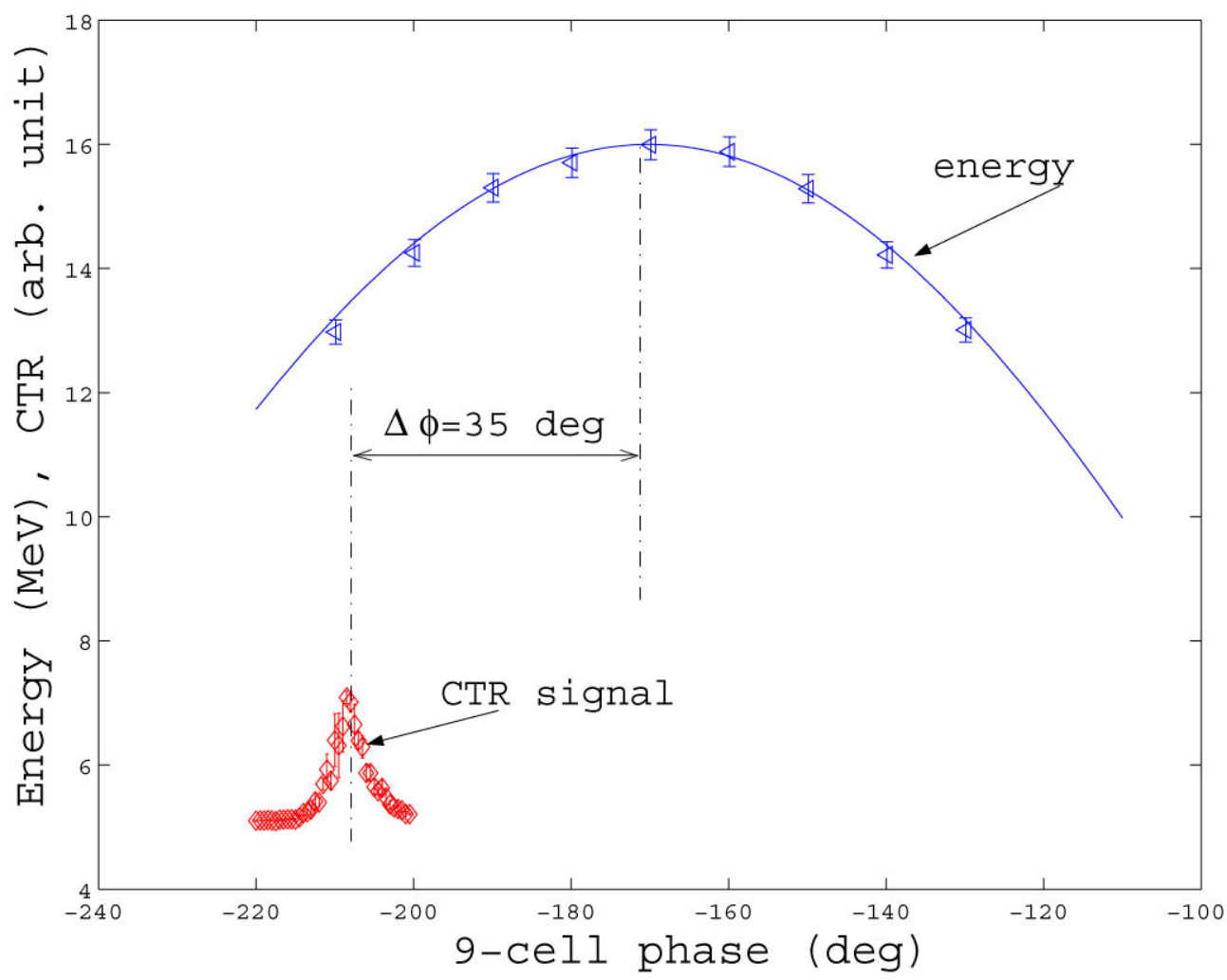

Figure 4.5: Energy of the electron bunch downstream of the 9-cell cavity as a function of the 9-cell phase. The CTR signal rises above the background only at the maximum compression phase, $\Delta \phi=35^{\circ}$. 

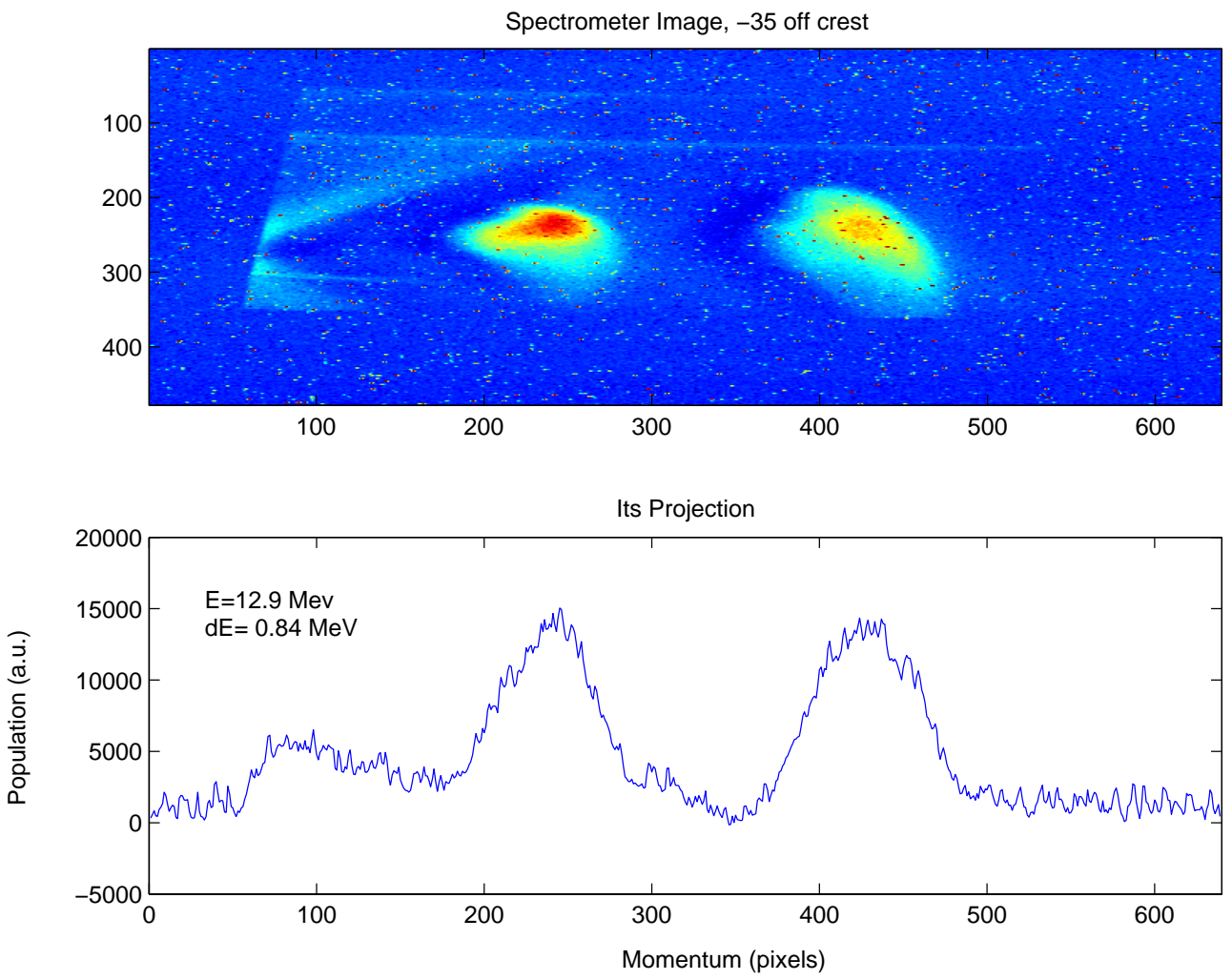

Figure 4.6: Measurement of the energy separation between the two macroparticles. 


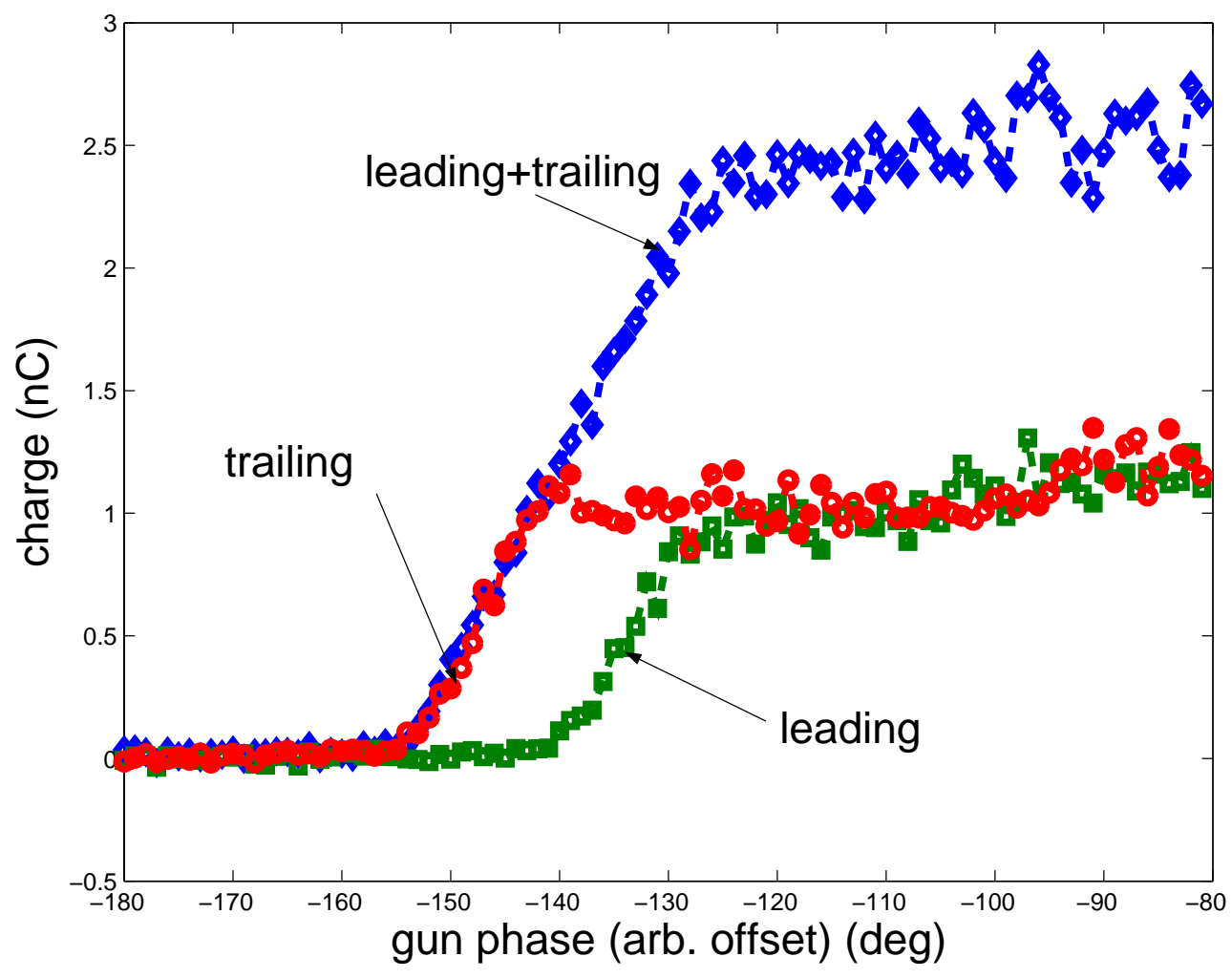

Figure 4.7: Scan of the phase between the rf-gun and the photocathode drive-laser when only leading (trailing) or both pulses are incident on the cathode. 
Table 4.1: Measured versus simulated $R_{56}$. The chicane current was $1.8 \mathrm{~A}$ (for the outer dipoles).

\begin{tabular}{|c|c|c|}
\hline Energy $(\mathrm{MeV})$ & $\left|R_{56}\right|$ measured $(\mathrm{cm})$ & $\left|R_{56}\right|$ simulated $(\mathrm{cm})$ \\
\hline 14.5 & $8.3 \pm 3.7$ & 7.47 \\
\hline 14.3 & $7.7 \pm 2.8$ & 7.82 \\
\hline 12.9 (max compression) & $9.1 \pm 1.1$ & 8.89 \\
\hline
\end{tabular}

$\left(-39^{\circ}\right.$ and $\left.-31^{\circ}\right)$ yield $6.3 \%$ energy difference, as compared to the measured value of $6.5 \%$.

Apart from the maximum compression case $\left(-35^{\circ}\right.$ off crest), we have also measured the value of $R_{56}$ for the 9-cell phase set of $\pm 20^{\circ}$ off crest. We compared it with the Matlab simulation for path length variation (see Eq. 4.42). The $R_{56}$ can be obtained by a linear fit as shown in Fig. 4.8. Despite the simple model used, the agreement between the calculated and measured values for $R_{56}$ is very good as summarized in Table 4.1.

\subsubsection{Velocity Bunching in RF-Gun and 9-cell Cavity}

The nominal rf-gun phase is set to $45^{\circ}$ with respect to the zero-crossing. The 9-cell cavity phase was adjusted to obtain the maximum energy gain. The two macropatricles were set to have a 20 ps optical path difference between the two laser pulses and the charge was approximately $1.5 \mathrm{nC}$ per macroparticle.

In the first experiment the rf-gun phase was varied while keeping the 9-cell cavity phase "on-crest". For each rf-gun phase the separation between the two macroparticles within the electron bunch was measured and then we computed the compression ratio by normalizing the measured time separation of the electron 

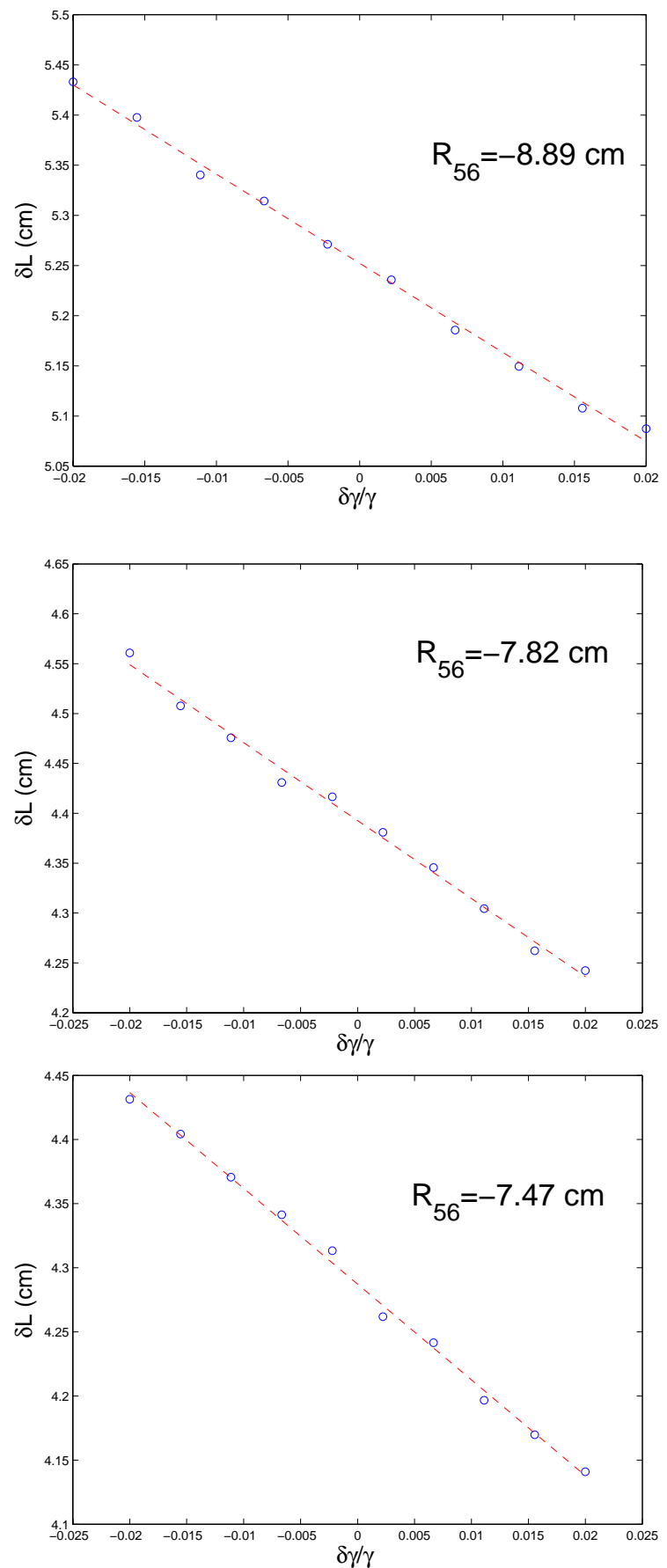

Figure 4.8: Calculated $R_{56}$ for three experimentally measured cases: $12.9 \mathrm{MeV}$ (top), 14.3 MeV (middle) and 14.5 MeV (bottom). 
bunches by the laser time separation: $\Delta \tau_{f} / \Delta \tau_{\text {cath }}$. The results are compared with ASTRA in Fig. 4.9.

In the second set of experiments, the 9-cell cavity phase was varied while keeping the rf-gun phase at its nominal value of $45^{\circ}$. Streak camera measurements of the time separation between the two macroparticles were made versus the cavity phase. The results are shown on Fig. 4.9. As before, the compression ratio is macroparticle time separation divided by the time separation when the cavity is operated on crest. Numerical simulations performed with ASTRA agree with the experimental measurement within the error bars. During this experiment we were limited to phases $\phi \in\left[-40^{\circ},+40^{\circ}\right]$ off-crest, trying to go further offcrest resulted in a large transverse envelope (due to rf-induced defocussing and chromatic aberrations).

\subsection{Conclusions}

The compression in a magnetic chicane, rf-gun and 9-cell cavity was studied using a novel Two Macroparticle method. The momentum compaction value for the chicane was found to be $8.2 \mathrm{~cm}$ (9.1 $\mathrm{cm}$ with higher order $\left(T_{566}\right)$ correction), which matches the simulated value of $8.9 \mathrm{~cm}$.

The velocity bunching effect was observed in both the rf-gun and the 9-cell cavity and is in excellent agreement with the ASTRA simulation.

The two macroparticle method could have promising applications as a method for direct probing of the longitudinal dynamics effects in a linac. Such applications could include commissioning of the free-electron laser, exploration of wakefield and coherent synchrotron radiation, and investigation of response time associated with photoemission. 

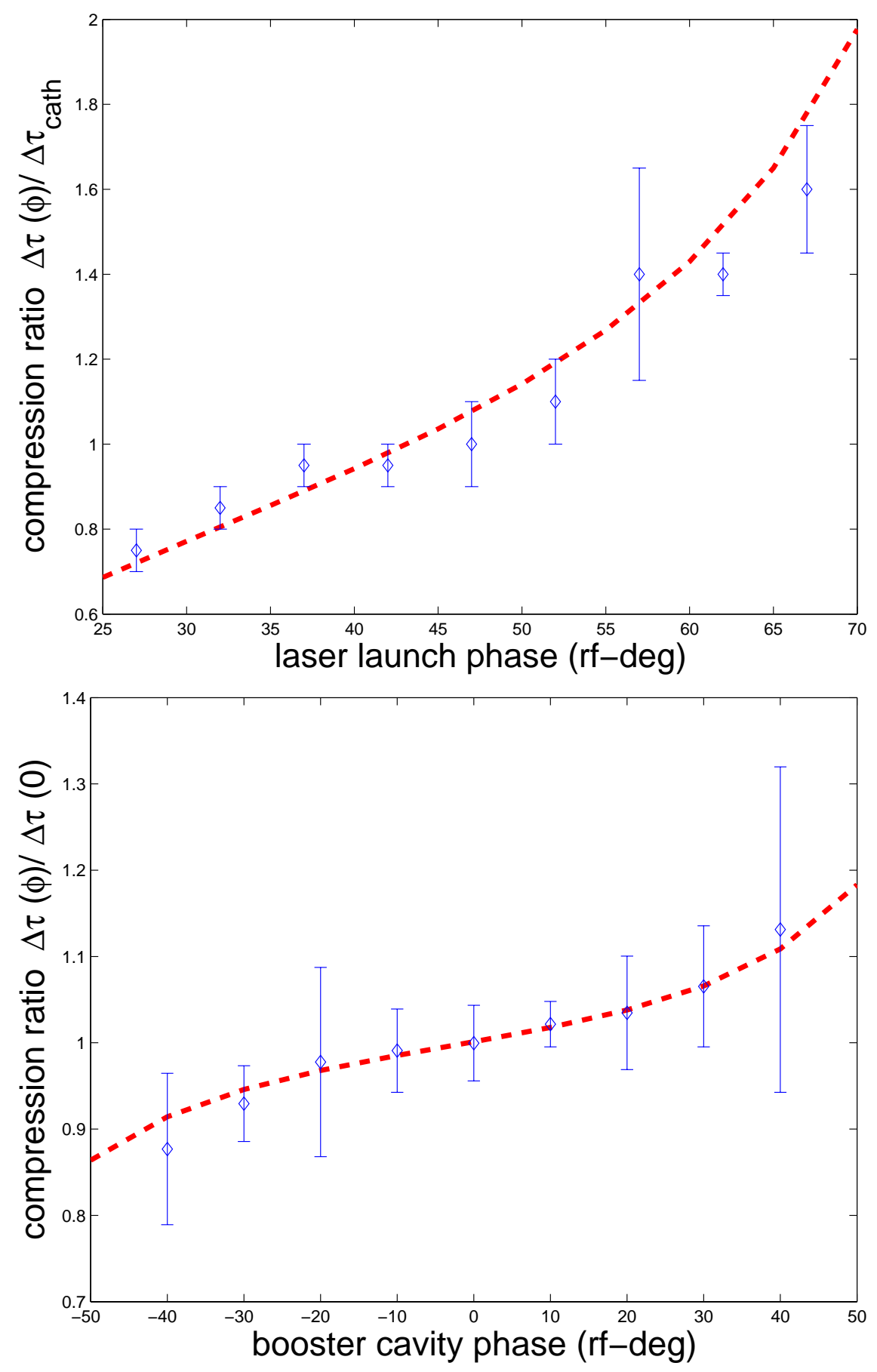

Figure 4.9: Velocity bunching in the rf-gun and 9-cell cavity. Compression ratio as a function of rf-gun phase (top) and 9-cell phase (bottom). Diamonds are experimental measurements and the dashed line corresponds to numerical simulations. 


\section{Chapter 5}

\section{Laser Acceleration of Electrons}

\section{$5.1 \quad$ Introduction}

In the last decades particle accelerators of ever increasing energy have been built and operated. All of these machines use rf technology. However, because of synchrotron radiation high energy electron colliders cannot be circular machines and this requires very high accelerating fields in excess of $40 \mathrm{MV} / \mathrm{m}$ if the machine is to be of finite length. Thus there has been a concerted effort to find alternate acceleration mechanism that can provide very high gradients. These involve acceleration by fields induced in plasmas and acceleration by focused short laser pulses [50]-[53].

All the laser acceleration schemes must provide a longitudinal component of the field and remain in synchronization (phase matched) with the electron bunch. In the rf regime this is achieved by propagating the rf power in a waveguide or similar structure. Such structures have dimensions of the order of the rf wavelength. For a laser field this would imply structures of dimensions of the order of one or few micrometers, which in turn, makes the tolerances on the electron beam size and 
position highly impractical.

In 1996 R. Pantel [50] proposed a scheme for propagating a laser beam in an open iris structure which is analogous to propagation in a Fabry-Perot resonator with flat mirrors. This scheme has been analyzed in detail by M. Xie [53] but has not as yet been tested. The phase velocity of the laser beam is only slightly in excess of the speed of light so that for a fully relativistic electron beam the phase matching length is $67 \mathrm{~cm}$. The maximum laser power that can be supported by the material of the structure is $34 \mathrm{TW}$. For this laser power the accelerating field is $E_{a}=0.54 \mathrm{GV} / \mathrm{m}[53]$.

It has been proposed to test this acceleration scheme with low energy electrons $(40 \mathrm{MeV})$ in which case a phase matching mechanism must be provided. One can load the structure with an inert gas to slow down the laser phase velocity. To estimate the accelerating field we note that

$$
I=\frac{U}{A \tau} .
$$

For pulse energy $U=2 \mathrm{~J}$ and pulse duration $\tau=2 \mathrm{ps}$ at $\lambda=1054 \mathrm{~nm}$ focused to an area of $A \sim 1 \mathrm{~mm}^{2}$, the peak intensity $I=10^{14} \mathrm{~W} / \mathrm{cm}^{2}$ resulting in the peak transverse field:

$$
E_{t}=\sqrt{2 Z_{0} I} \sim 10 \mathrm{GV} / \mathrm{m},
$$

where $Z_{0}=\sqrt{\mu_{0} / \epsilon_{0}}=337$ Ohms is vacuum impedance. Even though the corresponding peak acceleration gradient is more than two orders of magnitude smaller $\left(E_{a}=90 \mathrm{MV} / \mathrm{m}\right)$, it is still higher than any rf cavity can deliver. For a $0.5 \mathrm{~m}$ structure the energy gain would be $45 \mathrm{MeV}$, which is a spectacular change in momentum for a low energy injected beam.

According to the Lawson-Woodward-Palmer theorem [54], the laser-electron beam interaction region cannot extend to infinity but must be limited, and there 
always exists an inverse process that makes the electrons radiate. Since the interaction region will be filled with gas such a process would correspond to Cherenkov radiation. Thus the proposed scheme corresponds to Inverse Cherenkov acceleration.

\subsection{The Open Iris-loaded Waveguide Structure and Phase Matching}

\subsubsection{OILS}

An important advantage of an open iris-loaded structure (OILS) is its over-sized dimension compared to the laser wavelength. The structure consists of a number of stacked elements with a circular opening of radius a (See Fig. 5.1)[56].

Each element has tapered edges with the angle of tapering $\alpha_{T}$ greater than the diffraction divergence angle $\theta_{d}=\lambda / a$ so that the light sees it as an infinitely thin iris. The parameters of such a structure are given in Table 5.1. The structure

Table 5.1: Structure Parameters

\begin{tabular}{|l|l|}
\hline Parameter & Value \\
\hline Length & $\Lambda=10 \mathrm{~cm}(25 \mathrm{~cm})$ \\
\hline Diameter & $2 a=1 \mathrm{~mm}$ \\
\hline Number of Elements & $50(125)$ \\
\hline
\end{tabular}

can be visualized as an "unfolded" flat mirror Fabry-Perot resonator with Fresnel number:

$$
N=a^{2} / \lambda L=119
$$



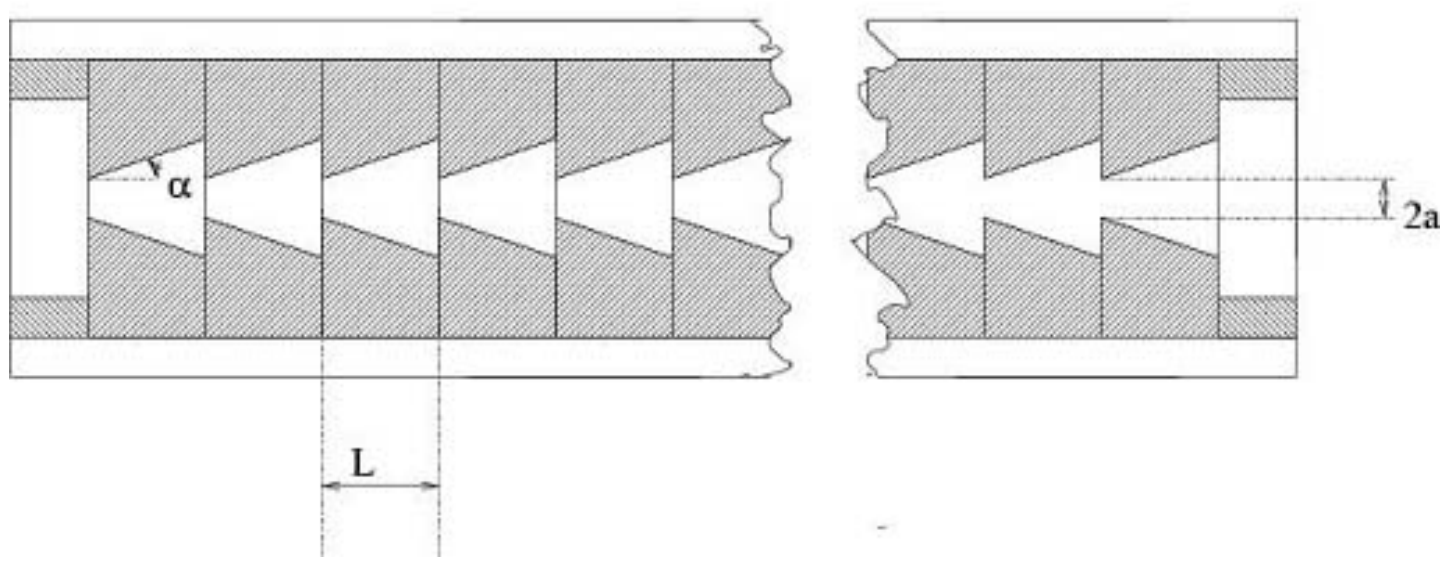

Figure 5.1: OILS Waveguide: radius $a=0.5 \mathrm{~mm}$, element length $L=2 \mathrm{~mm}$, angle of tapering $\alpha=20^{\circ}$.
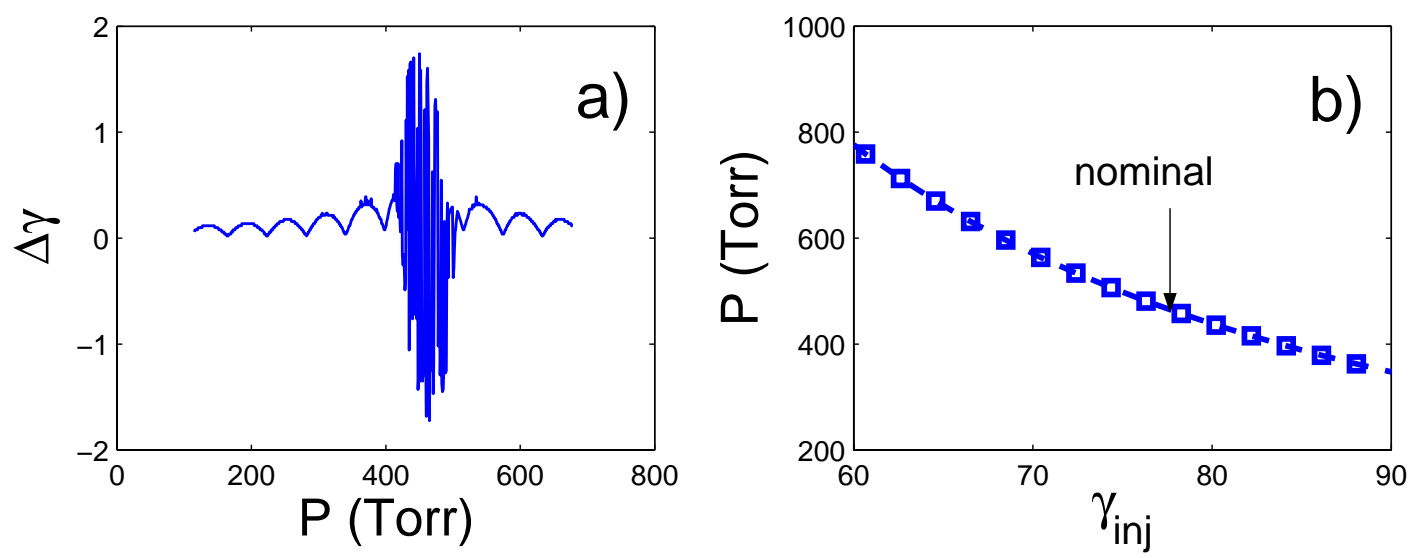

Figure 5.2: Sensitivity of energy gain on gas pressure (a) and required gas pressure for incoming energy (b). 


$$
Q=2 \pi L / \lambda \alpha_{c}=26 \times 10^{6}
$$

where $\alpha_{c}$ is the loss per cell;

$$
\alpha_{c}=8 v_{11}^{2}(M+\eta) \eta\left[(M+\eta)^{2}+\eta^{2}\right]^{2},
$$

where $v_{11}$ is the first zero of Bessel function:

$$
\begin{aligned}
& J_{1}\left(v_{11}\right)=0, \\
& v_{11} \approx 3.832 ; \\
& \eta=-\zeta(0.5) / \pi^{1 / 2},
\end{aligned}
$$

and $\zeta$ is Riemann's Zeta function;

$$
M=[8 \pi N]^{1 / 2} .
$$

Theoretical losses over a length $\Lambda=10 \mathrm{~cm}$ (and, later we will use $25 \mathrm{~cm}$ ) should be less than $5 \%(10 \%)$. It is interesting to note that such a large $Q$ factor allows the structure to be effective for a length of up to five kilometers.

\subsubsection{Phase Matching}

The eigenmodes of the structure were analytically derived by M. Xie [53]. The electric field associated to the TEM mode (no azimuthal dependence) is given by:

$$
\begin{aligned}
E_{z}(r, z, t) & =\hat{E} J_{0}\left(k_{r} r\right) e^{i\left(k_{z} z-\omega t\right)} \text { and } \\
E_{r}(r, z, t) & =\frac{k_{z}}{k} Z_{0} H_{\phi}(r, z, t) \\
& =-i \frac{k_{z}}{k_{r}} \hat{E} J_{1}\left(k_{r} r\right) e^{i\left(k_{z} z-\omega t\right)},
\end{aligned}
$$

where $\omega$ is the laser frequency in vacuum,

$\hat{E}$ is the axial peak electric field,

$k=n \omega / c$

$n$ is the index of refraction of the medium,

$$
k_{r}=v_{11} /(a[1+\eta(1+i) / M]) \text {, }
$$




$$
k_{z}=\sqrt{k^{2}-k_{r}^{2}} \simeq k-k_{r}^{2} /(2 k), \text { and }
$$

$Z_{0}$ is vacuum impedance.

Such a wave is supraluminous in vacuum: the phase velocity is

$$
v_{\phi} \equiv \frac{\omega}{\mathcal{R} e\left(k_{z}\right)} \simeq \frac{c}{n}\left[1+\frac{1}{2}\left(\frac{v_{11} \lambda}{2 \pi a}\right)^{2}\right],
$$

which is always larger than $c$ in vacuum $(n=1)$. This is a limitation, since any electron beam, especially non-ultra-relativistic incoming beams, such as considered in the present work, will eventually slip in phase. The laser phase velocity needs to be reduced by selecting a medium with the proper refractive index, e.g. immersing the interaction region in a gas. In order to have a net electron energy change, the laser and electron beams must be synchronous, i.e. the phase velocity of the laser should match the electron beam velocity. Two causes may break the synchronous condition: (1) the incoming beam is non-ultra-relativistic, and (2) when the energy gain is too high there will be significant phase slippage between the laser and electron beams. To avoid these effects, the laser phase velocity is decreased by immersing the interaction region in a gas with index of refraction chosen to have:

$$
n-1=\frac{1}{2}\left[\left(\frac{v_{11} \lambda}{2 \pi a}\right)^{2}+\frac{1}{\gamma^{2}}\right] .
$$

From past experience [57] with electron beam having similar parameters, filling the interaction region with hydrogen would be the best alternative. For Hydrogen $\left(\mathrm{H}_{2}\right)$, the index of refraction, at a given wavelength $\lambda$, is parameterized in term of gas pressure and temperature via the relation:

$$
n-1=10^{-6} \frac{P}{760} \frac{273.15}{T}\left(21.113+\frac{12723.2}{111-\lambda^{2}}\right)
$$

with $P, T$ and $\lambda$ respectively given in units of Torr, Kelvin and microns. For the nominal energy $\gamma=78$ we estimate from Eq. 5.5 for the refractive index $n-1 \simeq 8.3 \times 10^{-5}$, corresponding to a pressure of $\mathrm{H}_{2}$ of 450 Torr (see Fig. 5.2). 
Since the index of refraction depends on the gas pressure, pressure control can be used for fine-tuning of the structure. Note however, that the gas should not break down in the intense laser field. Note also that in the low energy regime as the electrons are accelerated the gas pressure must be reduced accordingly.

\subsection{Experimental Set-up}

\subsubsection{Laser System}

To obtain the desired laser field we need to use a regenerative Nd:glass laser with $\lambda=1054 \mathrm{~nm}$ seeded by an oscillator that has a long pulse length (200 ps), so that it is safe for the optics to generate $20 \mathrm{~mJ}$ pulses. After amplification the laser pulse would be compressed to a 2 ps width.

\section{Oscillator}

Our group designed and built the cw-pumped, mode-locked oscillator (see Fig. 5.3). It is based on the Quantronix 116 pump chamber that houses a $4 \mathrm{~mm}$ diameter by $79 \mathrm{~mm} \mathrm{Nd:YLF} \mathrm{rod.} \mathrm{The} \mathrm{cavity} \mathrm{consists} \mathrm{of} \mathrm{two} \mathrm{mirrors,} \mathrm{a} \mathrm{curved}$ ( $R=5 \mathrm{~m}$ ) high reflectivity and a flat output coupler (80\% transmission). Since the Nd:YLF lasing material can produce two wavelengths, $1047 \mathrm{~nm}$ and $1053 \mathrm{~nm}$, which possess mutually orthogonal polarizations (related to the ordinary and extraordinary axes of the YLF crystal), we introduce a Brewster plate polarizer to select the $1053 \mathrm{~nm}$ wavelength (s-polarized); this wavelength matches well the Nd:glass lasing wavelength $(1054 \mathrm{~nm})$ which is used in re-gen. To synchronize the oscillator with the rf system, we use an active mode-locking scheme based on the acousto-optic effect: the index of refraction in a medium is modified by the stress field of sound pressure wave. The oscillator produces the train of pulses with the 

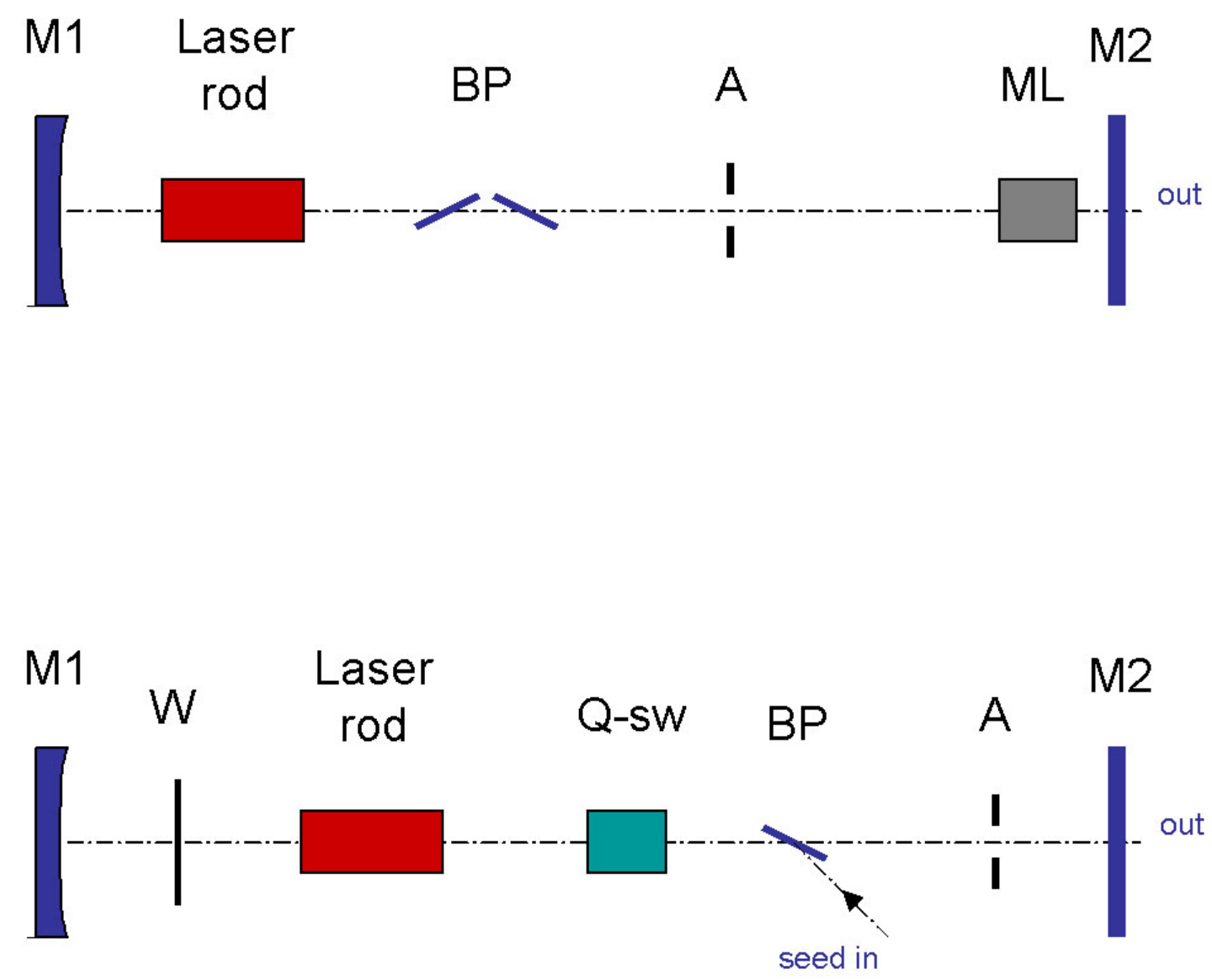

Figure 5.3: Schematics of the oscillator (top) and re-gen amplifier (bottom). M1, high reflectivity curved mirror; M2, output coupler (80\% transmission mirror); BP, Brewster polarizer; A, aperture; ML, mode locker; Q-sw, Q-switch Pockel cell; W, wire (to suppress the fundamental mode). 
repetition rate corresponding to the rf system $(81.25 \mathrm{MHz})$; the average power is $0.8 \mathrm{~W}$; the bandwidth is $5 \AA$ and the pulse duration is about 200 ps.

\section{Chirp Pulse Expansion and Compression}

We use a $2 \mathrm{~km}$ single mode fiber to produce a frequency chirp (linear correlation of the frequency and the length of the pulse), so that we can avoid high peak intensity of the laser beam during amplification and then to compress it using a grating compressor. This method is referred to as the chirp pulse amplification (CPA) technique [55]. The laser pulse passing through the fiber encounters two physical effects: self-phase modulation, which results in bandwidth broadening, and group velocity dispersion (GVD). The GVD effect results in pulse elongation and frequency modulation (chirp) which can be expressed as

$$
\beta \cong \frac{\lambda^{3}}{2 \pi c^{2}} \frac{d^{2} n}{d \lambda^{2}}
$$

where $n$ is the fiber refractive index, $\lambda$ is the laser wavelength, $c$ is the speed of light. After passing through the fiber the bandwidth is broadened to $25 \AA$, the pulse length is about 400 ps. We observe a $20 \%$ transmission efficiency.

We use a double-pass pair of gold-coated gratings to compress the beam after amplification. The GVD in the gratings introduce an "inverse" chirp

$$
\frac{d t}{d \lambda}=\frac{2}{c} \frac{(\lambda / d)(L / d)}{\cos ^{3} \theta_{\text {out }}}=\frac{2}{c} \frac{(\lambda / d)(L / d)}{\left[1-\left(\sin \theta_{\text {in }}-\lambda / d\right)\right]^{3 / 2}},
$$

where $L$ is the grating spacing and line density $d^{-1}=1700$ lines $/ \mathrm{mm}$. We can make the gratings and fiber chirps to match by adjusting the spacing $L$ (or the angle $\left.\theta_{\text {in }}\right)$. We obtained 2 ps pulselength after compression. 


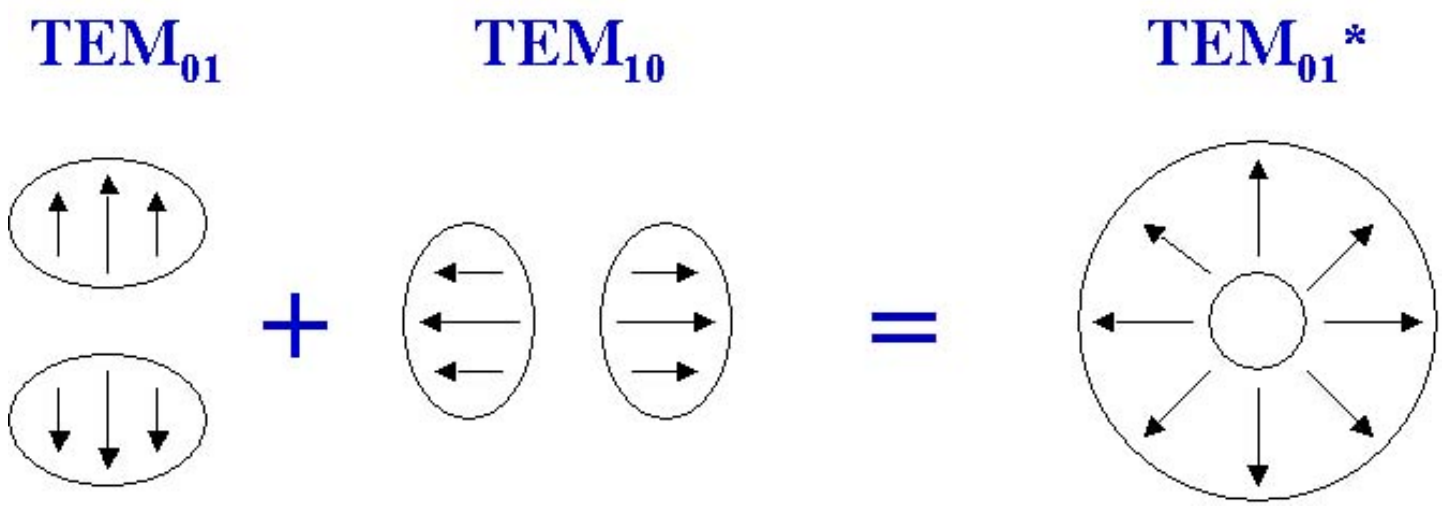

Figure 5.4: Doughnut shape mode $\left(T E M_{01}^{*}\right)$ is achieved as a linear combination of $\left(T E M_{01}\right)$ and $\left(T E M_{10}\right)$ modes with proper phase-matching.

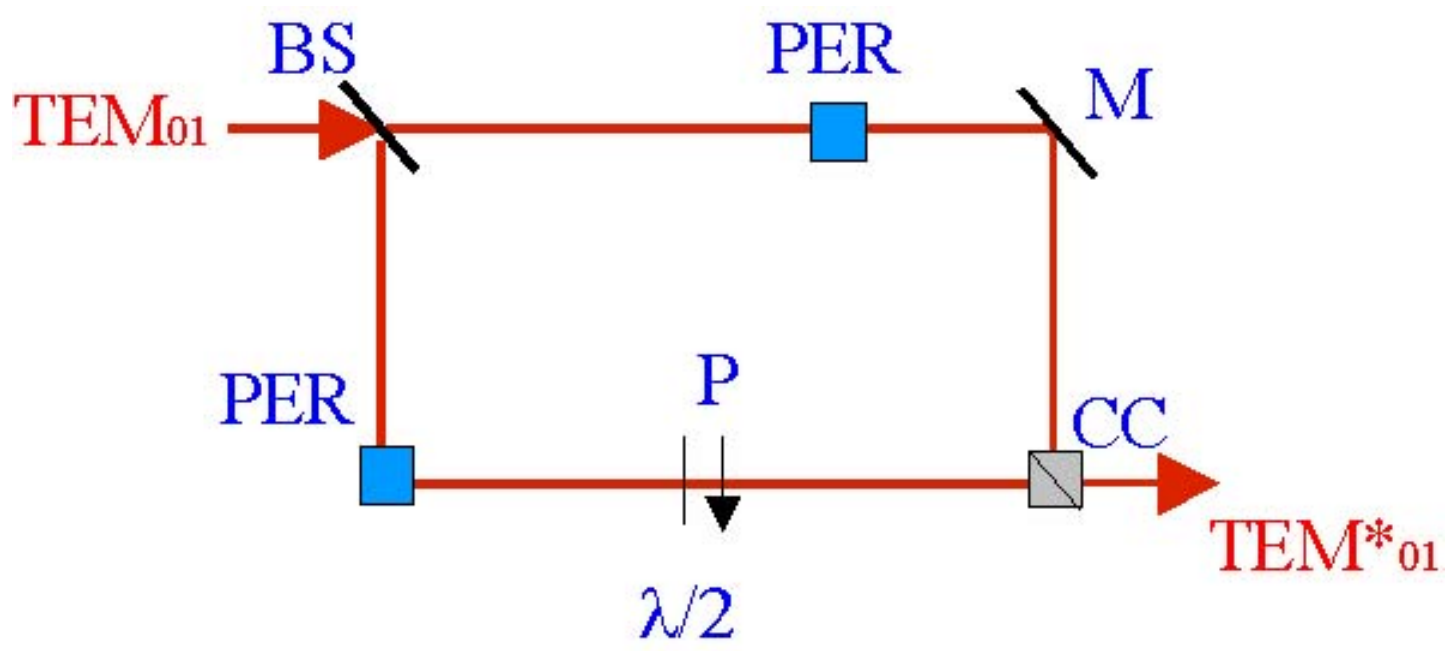

Figure 5.5: Mach-Zender Interferometer: 50/50 beam splitter $B S$, periscopes $P S 1$ and $P S 2$, combining cube $C C$, polarizer $P$, half-wave plate $P l$, piezo-driven mirror $M$. 


\section{Regenerative Amplifier}

We built the re-gen and produced a $T E M_{01}$ mode with stable preliminary parameters (1 mJ, $200 \mathrm{ps}$ ). The re-gen scheme (see Fig. 5.3) is analogous to the multi-pass discussed in section 2.3, brought to saturation. The Kigre laser head (model E40256KK) and power supply (model LCS 500) were used.

To generate the $T E M_{01}$ mode not only do we need to tune the cavity mirrors appropriately, but also we need to suppress the unwanted modes. To suppress the fundamental mode $\left(T E M_{00}\right)$ we introduce a thin wire next to a cavity mirror. This wire coincides with the node (dark central part) of the $T E M_{01}$ mode, thus causing no loss. To suppress higher order modes we use the usual technique of closing the intracavity irises to the appropriate size.

\subsubsection{Mach-Zender Interferometer}

For symmetry reasons and to gain a factor of $\sqrt{2}$ in accelerating field for given laser power, it is desirable to use radial polarization of the laser. A radially polarized field is shown in Fig. 5.4, and this mode is known as the $T E M_{01}^{*}$ mode (doughnut-shape).

One method for obtaining the $T E M_{01}^{*}$ mode is indicated in Fig. 5.5. We extract a $T E M_{01}^{*}$ mode from the laser and split it into two beams (50/50 beam splitter $B S)$. One beam is rotated by $90^{\circ}$ in periscope $P S 2$ and the two beams are then recombined (in beam cube $C C$ ) with the proper phase relationship; to compensate for the height difference of the two arms of the interferometer a second periscope $P S 1$ is used. To compensate for possible intensity difference, the combination of polarizer $(P)$ and half-wave plate $(P l)$ is used; to make sure that the beams 

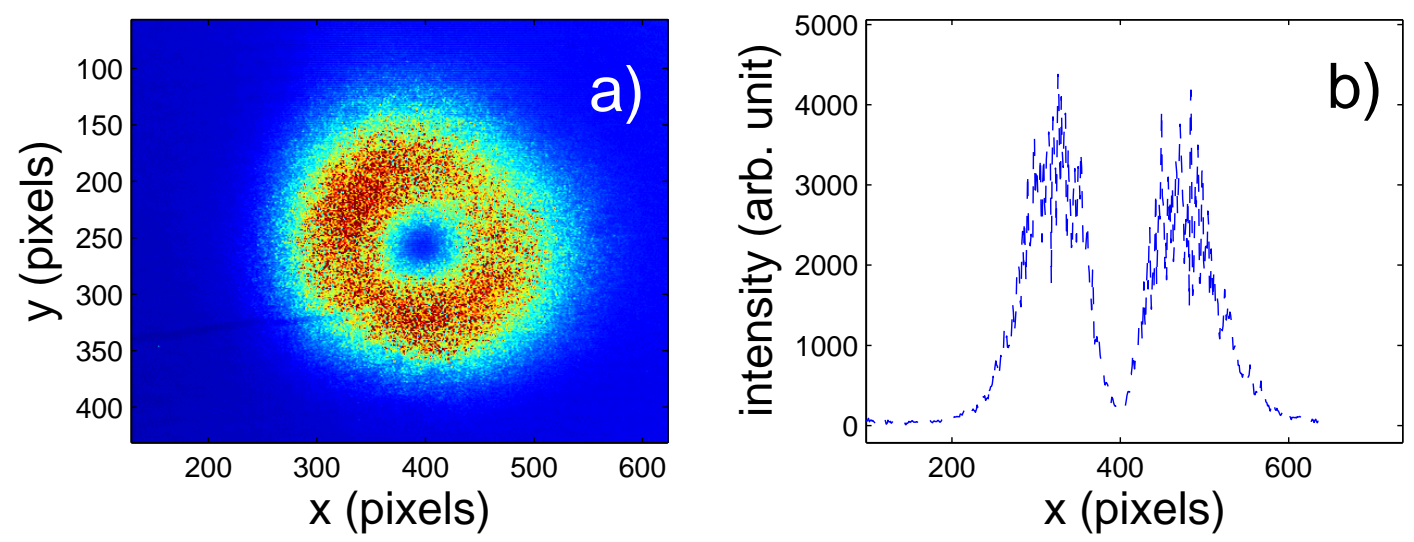

Figure 5.6: Measured transverse density a) and corresponding line profile b) of the $\mathrm{TEM}_{01}^{*}$ mode.

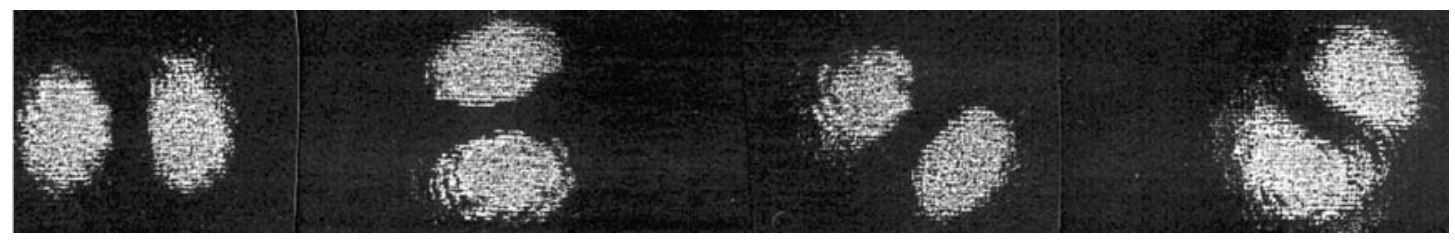

Figure 5.7: The phase matching test: polarizer oriented at different angles $(0,45$, 90 and 135 degrees). The polarizer makes the doughnut-shape mode $\left(T E M_{01}^{*}\right)$ collapse back to the $T E M_{01}$ mode losing its cylindrical symmetry; its orientation should correspond to the polarizer orientation for all angles. 
recombine in phase we use a piezo-driven mirror $(M)$. If the phase of the two interfering waves are not matched one obtaines a non-radial mode.

We have successfully built the interferometer and got the doughnut-shape mode (Fig. 5.6); we have also tested it for phase matching using a polarizer oriented at different angles (Fig. 5.7). The polarizer makes the doughnut-shape mode ( $T E M_{01}^{*}$ ) collapse back to the $T E M_{01}$ mode losing its cylindrical symmetry; its orientation should correspond to the polarizer orientation for all angles.

For efficient coupling between the $\mathrm{TEM}_{01}^{*}$ mode and the eigenvalue mode of the structure, we need to focus the mode to a waist $w \simeq 0.6 a$ where $a$ is the iris radius.

A $10 \mathrm{~cm}$ long structure was built and we obtained 85\% (intensity) transmission through the structure. The mode-structure of the beam remains the same before and after the waveguide. In fact there is no divergence of the beam associated with the structure - it acts like a weekly focusing lens sufficient to overcome the natural divergence of the Gaussian beam.

The preliminary design of the chamber is shown in Fig. 5.8. Aluminum or titanium foil would be used as windows of the chamber (being able to withstand up to 2 atm of inert gas). An uncompressed electron beam (2 ps rms) should be used. The electron beam should be focused (to $200 \mathrm{~mm}$ ) and collimated to $50 \mathrm{~mm}$. The expected accelerating field is of the order of $10 \mathrm{MV} / \mathrm{m}$ for the $20 \mathrm{~mJ}$ laser pulse. 


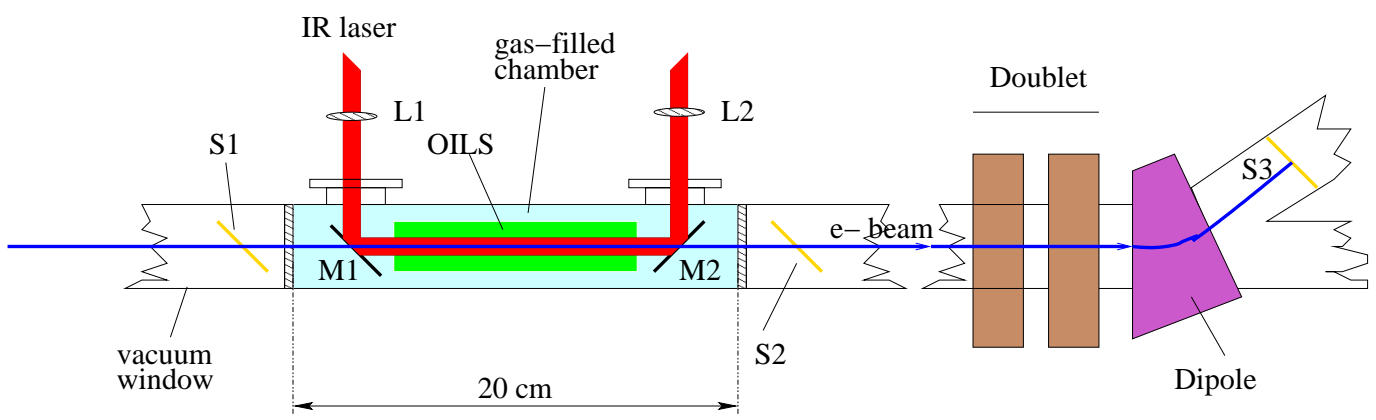

Figure 5.8: The laser beam enters from the top left. After propagating passing through a lens (L1) and reflecting from the apertured mirror (M1), the beam copropagates with the electron beam through the open iris-loaded structure (OILS) [green rectangle]. The laser beam is then extracted from the chamber thanks to a second apertured mirror (M2) and then transported to a diagnostics station.
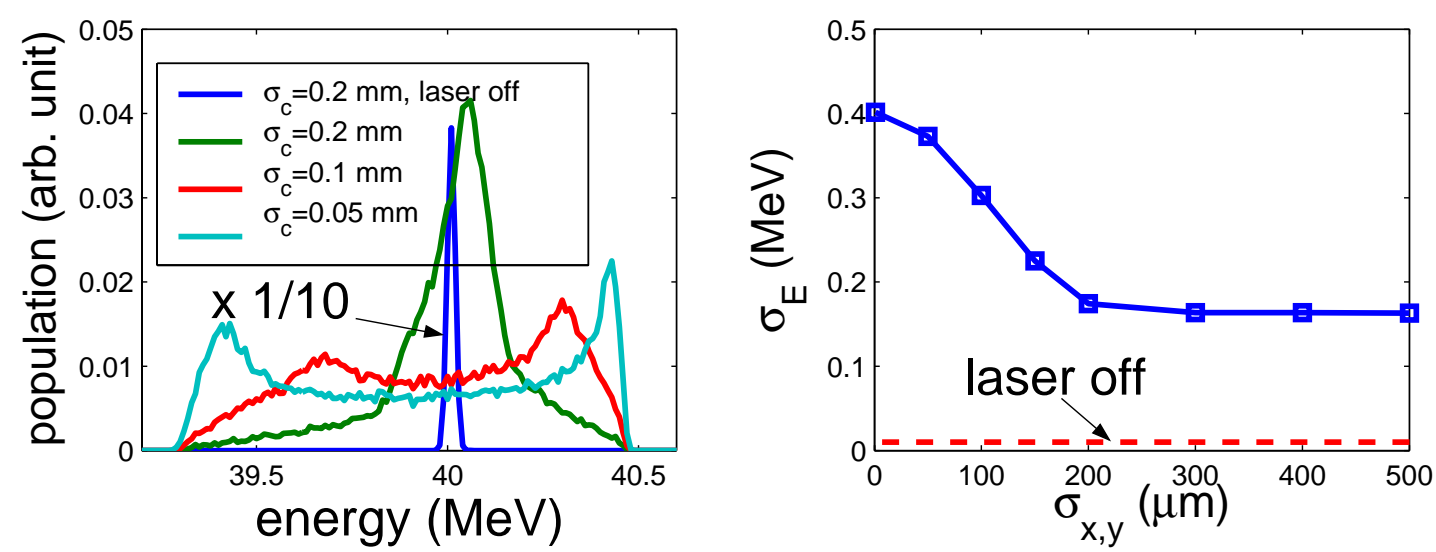

Figure 5.9: Simulated energy spectrum for various electron beam sizes in the OILS structure (left) and corresponding energy spread (right). Note for $\sigma_{x, y}>$ $0.2 \mathrm{~mm}$, the beam is not fully transmitted $\left(\sigma_{E}=10 \mathrm{keV}\right)$. (Note that the profile corresponding to the "laser off" case has been scaled by $1 / 10$ ). 


\subsection{Numerical Simulations}

We assume for the subsequent calculations of the laser acceleration performance a $40 \mathrm{MeV}^{1}$ electron beam. At the location of the interaction, we need an electron beam focused to transverse spot sizes $\sigma_{x}=\sigma_{y} \simeq 200 \mu \mathrm{m}$ (rms). Such a small focus insures that the beam is contained not only within the structure aperture (limited by the iris radius $a=1 \mathrm{~mm}$ ), but also in the region of high accelerating field (Fig. 5.9). Therefore we must optimize the electron transport to produce a low emittance beam. Since the charge per bunch is not a limiting parameter, we optimize the accelerator at the charge $Q=100 \mathrm{pC}$, low enough to achieve transverse normalized emittance below $1 \mathrm{~mm}$-mrad and, large enough to allow the use of our diagnostics (optical transition radiation and scintillating YAG screens). The main parameters of the low charge settings for the injector are reported in Table 5.2. For our estimate of the laser acceleration we consider an OILS with active length $L=10 \mathrm{~cm}$ and iris radius $a=1 \mathrm{~mm}$, the anticipated peak accelerating field is $\hat{E}=9 \mathrm{MV} / \mathrm{m}$.

Table 5.2: A0 nominal operating settings and simulated parameters for $100 \mathrm{pC}$ bunch.

\begin{tabular}{|c|c|c|}
\hline Parameter & Value & Unit \\
\hline charge & 100 & $\mathrm{pC}$ \\
\hline total energy & 43 & $\mathrm{MeV}$ \\
\hline transverse emittance & 0.7 & $\mathrm{~mm}-\mathrm{mrad}$ \\
\hline bunch length & $0.5(1.7)$ & $\mathrm{mm}(\mathrm{ps})$ \\
\hline momentum spread & 5.5 & $\mathrm{keV}$ \\
\hline
\end{tabular}

\footnotetext{
${ }^{1}$ This energy could be achieved after adding one more 9-cell cavity to the present A0 beamline.
} 
The two major effects that may degrade the expected signature of the laser/electron interaction are (1) multiple scattering as the beam crosses the vacuum window and propagate in the gas and (2) the effect of wakefields as the beam's Coulomb field interacts with the structure. The use of a gas at significant pressure (order of $1 \mathrm{~atm})$ to slow the phase velocity of the laser will deteriorate the electron beam via beam-gas scattering. The total emittance growth due to scattering is approximately $\Delta \tilde{\varepsilon}_{x} \simeq \sum_{i} 2 / 3(28 / \gamma)^{2}\left[L^{(i)} / X_{0}^{(i)}\right]$, where the summation is performed on the different constituents degrading the beam (two beryllium windows and the gas volume), $X_{0}$ and $L$ are the radiation length and interaction length. Our estimate results in a beam inflation by a factor of $\sim 2$ at the end of the structure. Even though the exited longitudinal wake-field would cause the bunch energy spread to grow, this effect is indeed small at $100 \mathrm{pC}$ for the considered bunch duration $\left(\sigma_{t} \simeq 2 \mathrm{ps}\right)$.

We used a modified tracking code ASTRA [42] to simulate laser acceleration. A new element was introduced into the program to accommodate a beam line section with a different phase velocity according to Eq. 5.3. The program then integrates the equation of motion taking into account space charge and the laser external fields. In the calculations reported here we do not include the beam-scattering due to the gas in the interaction region and entrance/exit vacuum windows. We also assume that the entire electron beam is interacting with the laser beam, namely the laser pulse in not shorter than the electron bunch.

The main signature of the laser/electron interaction occurs on the electron longitudinal phase space. Because the laser wavelength is much shorter than the bunch length, an energy modulation is transferred to the electron beam. The build-up of energy modulation and the associated density modulation are seen in Fig. 5.10. 

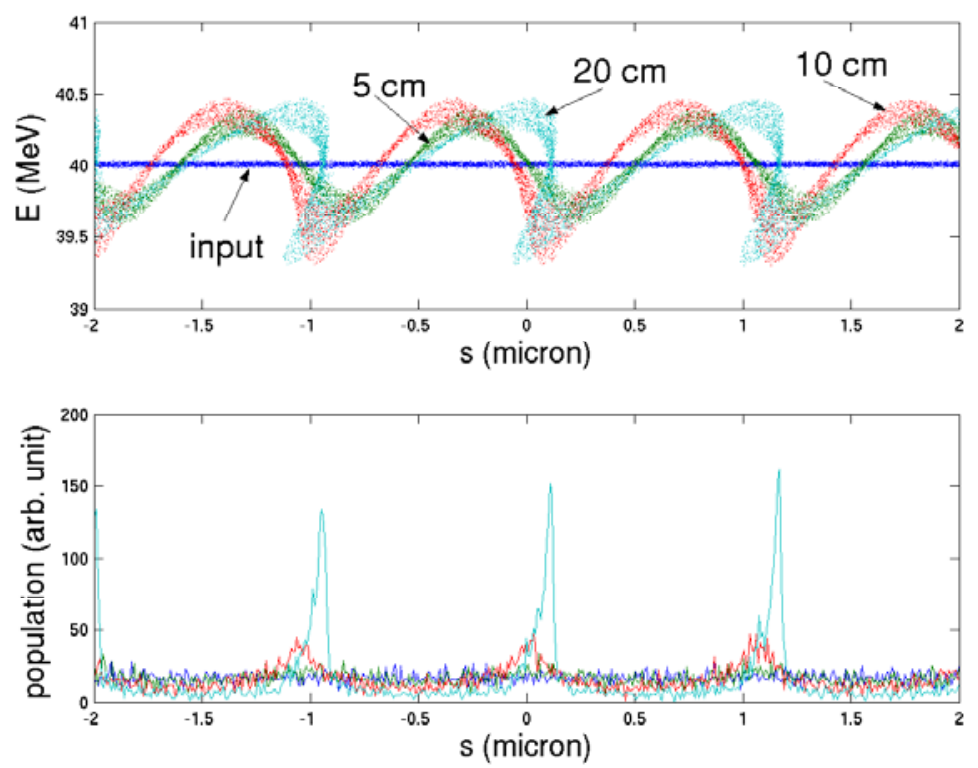

Figure 5.10: Energy and density modulation: (top) longitudinal phase space upstream (input), and at different locations downstream of the OILS section entrance $(5,10$ and $20 \mathrm{~cm})$ along with the associated charge density (bottom) $(s>0$ corresponds to the bunch head).

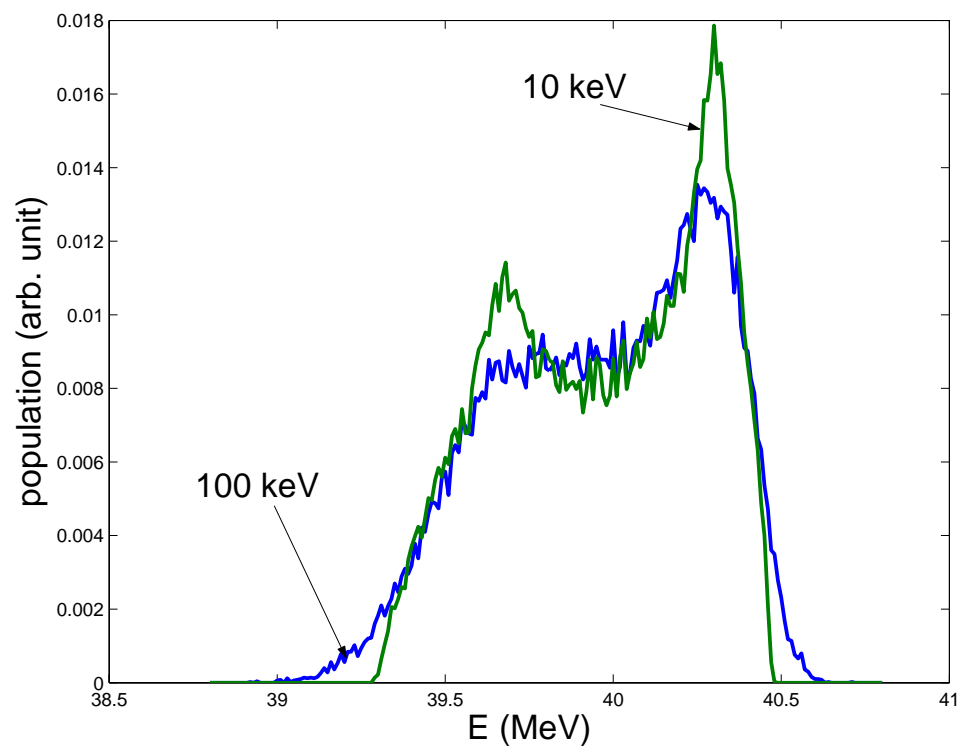

Figure 5.11: Sensitivity of the energy spectrum on the incoming electron beam energy spread $\left(\sigma_{x, y}=0.1 \mathrm{~mm}\right)$. 
This results in a double-peaked energy spectrum as shown in Fig. 5.11. The influence of incoming energy spread is not significant: even for large incoming energy spread (100 keV rms) the spectrum is not significantly altered. Because of the radial dependence of the longitudinal field we also investigate the evolution of energy spectrum in the OILS for various electron beam sizes (see Fig. 5.9). The results indicate that it is necessary to keep the beam sizes small $\left(\sigma_{x, y}<200 \mu \mathrm{m}\right)$ in order to avoid smearing of the double-peak structure. An advantage of accelerating non-ultra-relativistic electron beams is that the energy modulation eventually converts into a density modulation at the laser wavelength (micro-bunching). The maximum compression is achieved after a drift length $D=1 / \gamma^{2}(d \delta / d s)^{-1} \simeq 10 \mathrm{~cm}$ downstream of the OILS (where $d \delta / d s$ is the fractional energy chirp imparted by the laser). The micro-bunching could be used for other advanced accelerator physics applications (such as injection of pre-bunched beam in a plasma-wakefield accelerator); it could also produce coherent radiation, e.g., for bunch length diagnostics.

\subsection{Conclusion}

The $\mathrm{TEM}_{01}$ mode laser system has been developed and the preliminary requirements have been met. The doughnut-shape mode in the pulsed regime has been achieved for the first time using the Mach-Zender interferometer. The Open Irisloaded Waveguide structure has been built and tested. We have analyzed the possibility of performing an inverse Cherenkov acceleration experiment at A0 with a $40 \mathrm{MeV}$ electron beam. A clear signature of laser/electron interaction should be obtained in this experiment for a large range of incoming electron beam parameters. The experiment could be performed after the A0 upgrade [34]. 


\section{Bibliography}

[1] R.J. Van de Graaff, Phys. Rev. 38, p. 1919 (1931).

[2] D.W. Kerst, R. Serber, Phys. Rev. 60, p. 53 (1941).

[3] D. Edwards, M. Syphers, An Introduction to the Physics of High Energy Accelerators, Wiley and Sons (1993).

[4] J.M.J. Madey, J. Appl. Physics 42, p. 1906 (1971).

[5] D.A.G. Deacon, et al., Phys. Rev. Lett. 38, p. 892 (1977).

[6] J. Rossbach, Nucl. Instr. Meth. A 475, p. 13 (2001).

[7] Zeroth Order Design Report for the Next Linear Collider, SLAC-R-474 (1996).

[8] JLC Design Study, KEK-R-97-1 (1997).

[9] TESLA Technical Design Report (2001).

[10] H. Wiedemann, Particle Accelerator Physics, Springer-Verlag (1993).

[11] S.Y. Lee, Accelerator Physics, World Scientific (1999).

[12] J.P. Carneiro et al., proc. of XIX Int. Linac Conf., p. 642 (1998). 
[13] J. Andruszkow, et al., Phys. Rev. Lett. 85, p. 3825 (2000).

[14] Linac Coherent Light Source (LCLS) Design Study Report, SLAC-R-521 (1998).

[15] M.J. Fitch et al, Phys. Rev. Lett. 87, 034801 (2001).

[16] W. Hartung et al, proc. of PAC01, p. 2239 (2001).

[17] Y.-E Sun et al, Phys. Rev. ST A\&BB 7, 123501-1 (2004).

[18] Y.-E Sun Ph.D Dissertation, U Chicago (2005).

[19] N. Barov et al, Phys. Rev. ST A\&BB 7, 061301-1 (2004).

[20] M.C. Thompson et al, proc. of AIP conf., 737 (2004).

[21] A.R. Fry, M.J. Fitch, A.C. Melissinos, B.D. Taylor, Nucl. Instr. Meth. A 430, p. 180 (1999).

[22] G Gollin, private communications (2005). (information available on the web site http://www.hep.uiuc.edu/home/g-gollin/

[23] J. Li, R. Tikhoplav and A.C. Melissinos, FERMILAB-PUB-06-020-AD (2006).

[24] The laser specifications can be found on the website of the company: www.tbwp.com/Time_Bandwidth/Sites/S_Products/FrameSetPico/ GE100.htm

[25] Conoptics, Inc., m360-80 modulator and model 25D HV driver.

[26] I. Will et al, IEEE Quant. Elect. 34(10), p. 2020 (1998).

[27] I. Will et al, Nucl. Instr. Meth. A 541, p. 467 (2005). 
[28] P. Laporta, V. Magni, and L. Pallaro, Optics Comm. 51, p. 95 (1984).

[29] M.J. Fitch, Ph.D Dissertation, UR-1628 (2000).

[30] Hewlett-Packard HP70951A Optical Spectrum Analyzer on loan from R. Pasquinelli (FNAL).

[31] Conoptics, Inc., m360-105 modulator and model 25D HV driver.

[32] A.R. Fry, Ph.D Dissertation, UR-1492 (2000).

[33] F. Zhou, I. Ben-Zvi, M. Babzien, X. Y. Chang, A. Doyuran, R. Malone, X. J. Wang and V. Yakimenko, Phys. Rev. ST 5 094203-1 (2002).

[34] P. Piot et al, PAC05 paper TPPE044 (2005).

[35] S. Schreiber, TESLA Collaboration Meeting, WG3 (2004).

[36] J. Li, P. Piot, R. Tikhoplav, PAC05 paper WPAP043 (2005).

[37] J. Qiang et al., PAC05 paper WPAP055 (2005).

[38] D.A. Reis, Ph.D Dissertation, UR-1573 (1999).

[39] K. Flöttemann, TESLA-EFL 97-01 (1997).

[40] P. Piot, D.R. Douglas and G.A. Krafft, Phys. Rev. ST A $\mathscr{B} B 6030702$ (2003).

[41] K.-J. Kim, Nucl. Instr. Meth. A 275, 201-218 (1989).

[42] K. Flöttmann, ASTRA user manual, DESY (2000). (information available on the web site http://www.desy.de/^mpyflo)

[43] X. Qiu et al., Phys. Rev. Lett. 76, p. 3723 (1996).

[44] S. Giermann, Ph.D Dissertation, UC San Diego (2001). 
[45] B.E. Carlsten, Nucl. Instr. Meth. A 285, p. 313 (1989).

[46] Matlab is available from MathWorks, Inc.

[47] K.L. Brown et al., report CERN 73-16, (revised as CERN 80-4) CERN (1980).

[48] D. Mihalcea, private communications (2005).

[49] T.O. Raubenheimer, et al., proc. of PAC 1997, p. 635 (1997).

[50] R. Pantel, Nucl. Instr. Meth. A 393, p. 1 (1997).

[51] W.D. Kimura et al., Phys. Rev. Lett. 74, 546 (1995).

[52] Y.C. Huang et al., Appl. Phys. Lett. 68(6), 753 (1996).

[53] M. Xie, Berkeley reports LBNL-40558 (1997) and LBNL-42055 (1998).

[54] J.D. Lawson, IEEE Trans. Nucl. Sci. NS-26, p, 4217 (1979); P.M. Woodward, J. IEEE 93, p. 1554 (1947).

[55] P. Bado et al., Optics Letters 12(5), 319 (1987).

[56] R. Tikhoplav et al., proc. of EPAC 2002, p. 984 (2002).

[57] A. S. Fisher et al., Nucl. Instr. Meth. A 272, p. 89 (1988).

[58] W. Koechner, Solid-State Laser Engineering, Springer (1999). 


\section{Appendix A}

\section{Vibration Measurements}

\section{A.1 Introduction}

Output stability (both energy and pointing) is a crucial parameter for a laser system performance. It is especially important for the A0 photocathode drive laser, since firstly, it is a complex system (oscillator and two stages of amplification). Secondly, one of the amplification stages, the multipass, is not driven into saturation (like a re-gen), thus it amplifies the fluctuations as well. There are many more sources of laser instability, such as: air currents around optical elements, temperature fluctuations, electrical circuitry instabilities, vibrations of the optical tables. Here we present an attempt to quantitatively measure the vibration in the laser room in order to mitigate it in future installations.

\section{A.2 Description of the Measurements}

The vibration measurements at the A0 laser room were performed on the floor and on three optical tables: Oscillator (Table One), Multipass (Table Two) and 
Compression (Table Three). The measurements were carried out for both vertical and horizontal modes using low frequency $(0.05-200 \mathrm{~Hz})$ velocimeters of type SM3KV\#1429 (horizontal) and SM-3KV\#1434 (vertical). Refer to Table A.1 and Table A.2 for the results. In these tables entries with the asterisk indicate that a nearby air compressor was turned on. A typical oscilloscope trace is shown in Fig. A.1.

Table A.1: Vertical vibration, sensor \# 1434

\begin{tabular}{|c|c|c|c|c|c|c|c|c|c|}
\hline & \multicolumn{2}{|c|}{$17 \mathrm{~Hz}$} & \multicolumn{2}{|c|}{$30 \mathrm{~Hz}$} & \multicolumn{2}{|c|}{$40 * \mathrm{~Hz}$} & \multicolumn{2}{|c|}{$60 \mathrm{~Hz}$} & \multirow{2}{*}{ Resonance } \\
\cline { 2 - 10 } & $\mathrm{mV}$ & $\mathrm{nm}$ & $\mathrm{mV}$ & $\mathrm{nm}$ & $\mathrm{mV}$ & $\mathrm{nm}$ & $\mathrm{mV}$ & $\mathrm{nm}$ & frequency \\
\hline Floor & 30 & 3 & 30 & 2 & 90 & 4 & 30 & 1 & $\mathrm{~N} / \mathrm{A}$ \\
\hline Table One & 30 & 3 & 30 & 2 & 320 & 13 & 100 & 3 & $38 \mathrm{~Hz}$ \\
\hline Table Two & 30 & 3 & 30 & 2 & 150 & 6 & 30 & 1 & $\mathrm{~N} / \mathrm{A}$ \\
\hline Table Three & 20 & 2 & 20 & 1 & 220 & 10 & 120 & 4 & $45 \mathrm{~Hz}$ \\
\hline
\end{tabular}

The Oscillator table has an air-floating capability which has been successfully utilized. Floating the tables helps reduce the noise level and narrow the resonance range while shifting it to the lower frequency region. The latter corresponds to the movement of an optical table as a whole (thus the optical elements on the table do not move relative to each other) rather than exciting a wave on its surface. The results are given in Table A.3.

We have compared measurements at the Argonne National Lab Advanced Photo Source (APS) facility with the A0 facility at Fermilab. We found that the A0 facility was approximately three times noisier as compared to the APS (see Fig. A.2).

Two kinds of seismometers have been used in our APS/A0 comparative mea- 


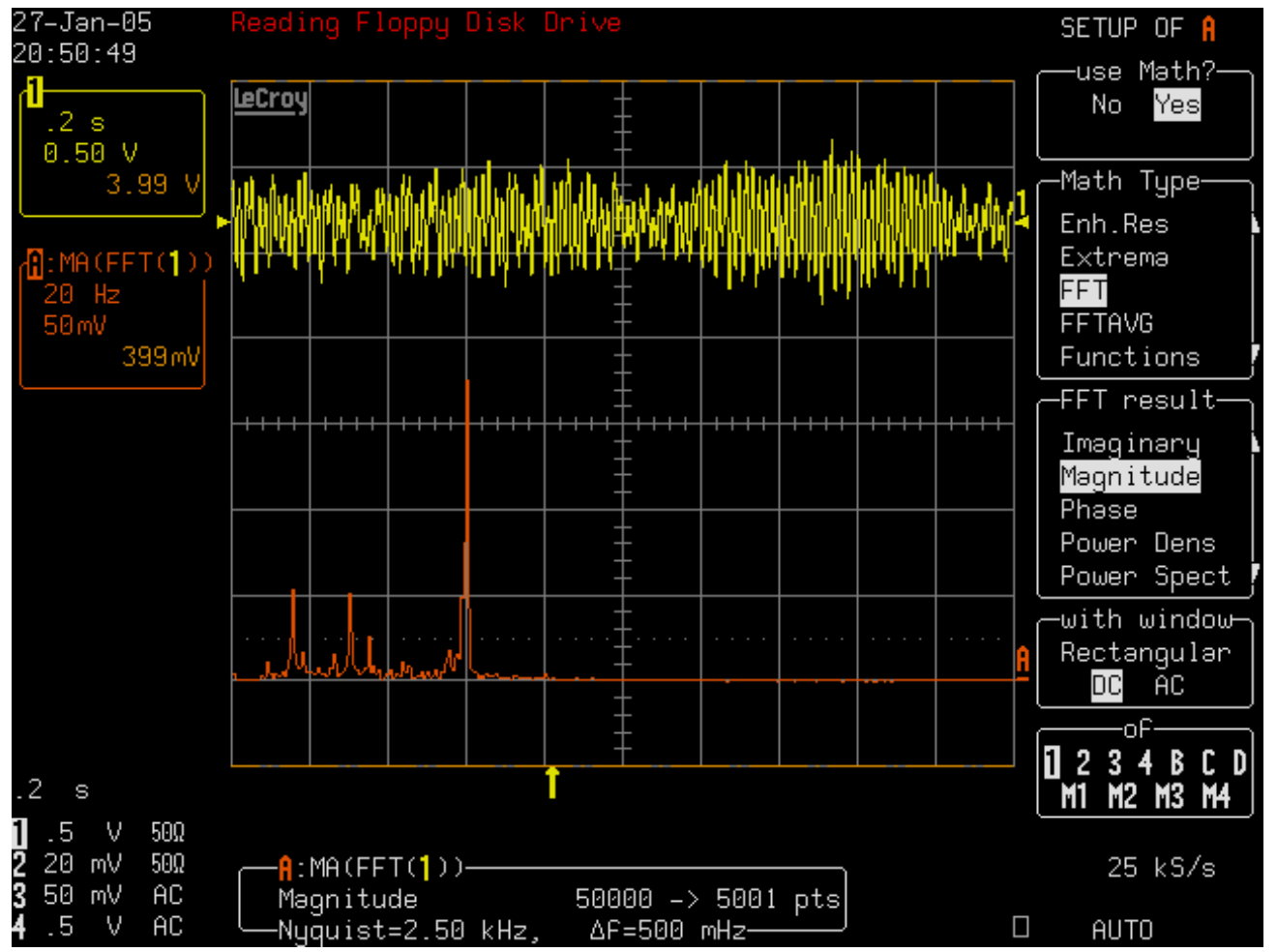

Figure A.1: A typical Lecroy oscilloscope trace of a velocimeter in the laser room. The upper trace corresponds to the signal, the lower to its FFT 
Table A.2: Horizontal vibration, sensor \# 1429

\begin{tabular}{|c|c|c|c|c|c|c|c|c|c|}
\hline & \multicolumn{2}{|c|}{$17 \mathrm{~Hz}$} & \multicolumn{2}{|c|}{$17^{*} \mathrm{~Hz}$} & \multicolumn{2}{|c|}{$40^{*} \mathrm{~Hz}$} & \multicolumn{2}{|c|}{$60 \mathrm{~Hz}$} & \multirow{2}{*}{$\begin{array}{c}\text { Resonance } \\
\text { frequency }\end{array}$} \\
\cline { 2 - 9 } & $\mathrm{mV}$ & $\mathrm{nm}$ & $\mathrm{mV}$ & $\mathrm{nm}$ & $\mathrm{mV}$ & $\mathrm{nm}$ & $\mathrm{mV}$ & $\mathrm{nm}$ & Low $<1 \mathrm{~Hz}$ \\
\hline Floor & 6 & 0.6 & 6 & 6 & 10 & 0.4 & 6 & 0.2 & Low \\
\hline Table One & 70 & 7 & 680 & 66 & 100 & 4 & - & - & $15 \mathrm{~Hz}$ \\
\hline Table Two & 60 & 6 & 500 & 48 & - & - & - & - & $15 \mathrm{~Hz}$ \\
\hline Table Three & 80 & 8 & 590 & 57 & 30 & 1 & 10 & 0.3 & $15 \mathrm{~Hz}$ \\
\hline
\end{tabular}

surements: velocimeter type and accelerometer type. The velocimeter principle of operation is based on the motion of a magnetic spring pendulum in a solenoid. The amplitude and frequency spectrum of the current in the solenoid directly reflect the amplitude and frequency spectrum of the vibrations. The accelerometer is a piezo-type device which measures the force exerted on the piezo crystal and thus measures by virtue of the Newton's second law of motion, the acceleration induced by the vibration. Accelerometers are best suited for higher frequencies (greater than $100 \mathrm{~Hz}$ ).

Table A.3: Oscillator Table (One) when floated

\begin{tabular}{|c|c|c|c|c|c|c|c|c|c|}
\hline & \multicolumn{2}{|c|}{$1.8 \mathrm{~Hz}$} & \multicolumn{2}{|c|}{$5 \mathrm{~Hz}$} & \multicolumn{2}{|c|}{$5^{*} \mathrm{~Hz}$} & \multicolumn{2}{|c|}{$12^{*} \mathrm{~Hz}$} & \multirow{2}{*}{$\begin{array}{c}\text { Resonance } \\
\text { frequency }\end{array}$} \\
\cline { 2 - 9 } & $\mathrm{mV}$ & $\mathrm{nm}$ & $\mathrm{mV}$ & $\mathrm{nm}$ & $\mathrm{mV}$ & $\mathrm{nm}$ & $\mathrm{mV}$ & $\mathrm{nm}$ & $5 \mathrm{~Hz}$ \\
\hline Vertical & - & - & 40 & 15 & 50 & 19 & - & - & 50 \\
\hline Horizontal & 20 & 20 & 10 & 4 & 10 & 4 & 50 & $1.8,2.5,5 \mathrm{~Hz}$ \\
\hline
\end{tabular}




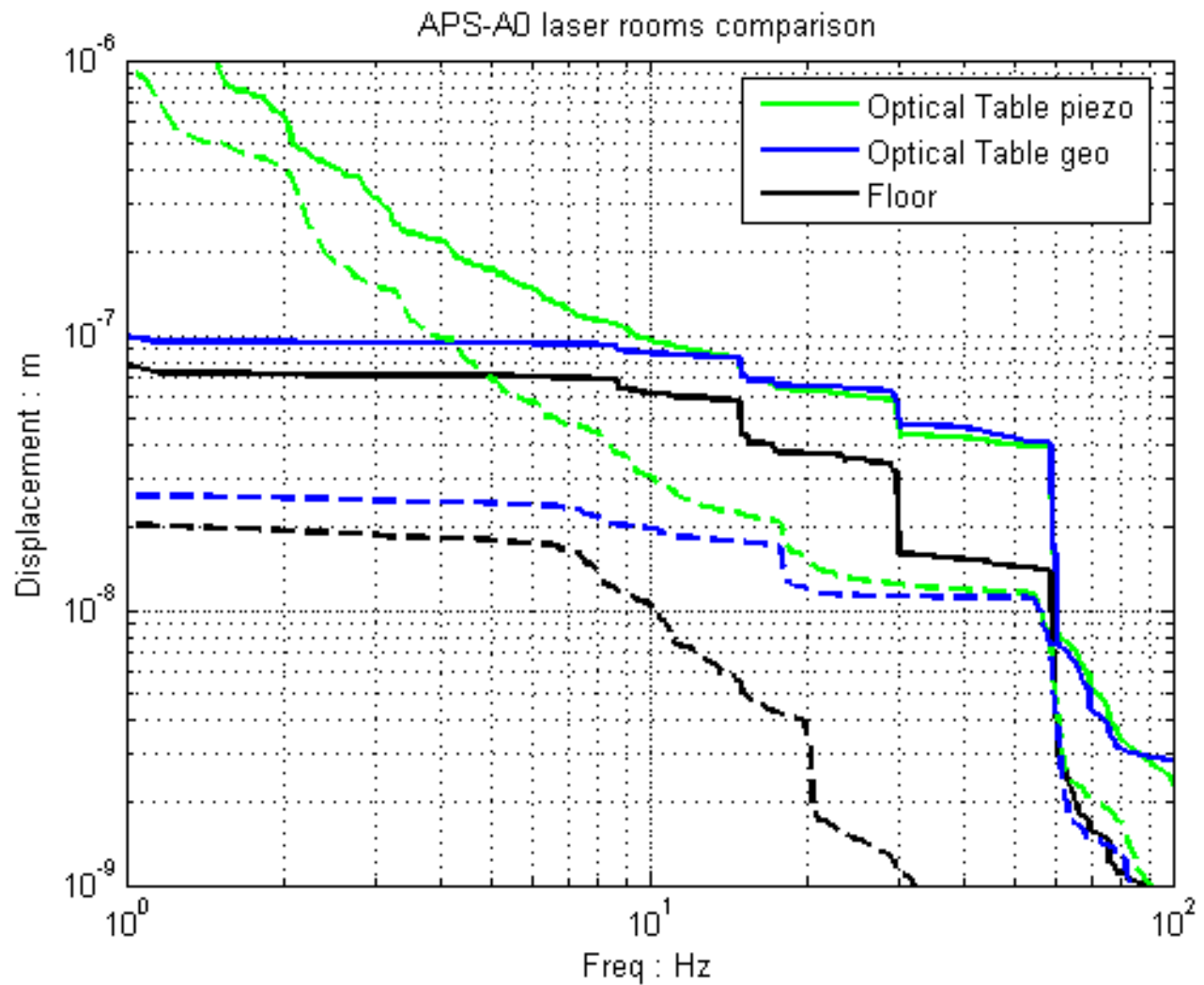

Figure A.2: Integrated vibration measurements in the A0 (solid line) and APS (broken line) facilities. Integration goes from high to low frequency. "Piezo" and "geo" stand for the accelerometer and velocimeter measurements respectively. Floor was measured by velocimeter only. 


\section{A.3 Analysis of the Results}

The overall integrated vibration amplitude for the worst conditions is about one tenth of a laser wavelength (which is $1 \mu \mathrm{m}$ ), so to the first approximation the vibration effect is not very significant. However, we can reduce the noise level by as much as a factor of ten and be comfortably far away from the scale of laser wavelength by removing an air-compressor situated right behind the laser room wall (see Tables A.1 and A.2 ).

Although it helps reducing the noise level, floating the tables is not very practical. The system becomes very sensitive to operator motion in the laser room, and a simple touch of the table initiates resonant oscillations. Thus we decided against floating the tables. 


\section{Appendix B}

\section{Analysis of Frequency Doubling Efficiency}

\section{B.1 Introduction}

The A0 seed oscillator generates a train of laser pulses at wavelength $\lambda=1054 \mathrm{~nm}$. Such a train is then amplified by the multi-pass and 2-pass amplifiers. In order to extract electrons from the cesium telluride photocathode, the photons need to have an energy $\hbar \omega \geq 3.5 \mathrm{eV}$, which corresponds to a wavelength $\lambda<353 \mathrm{~nm}$. The IR photons can be used to generate their 2nd and 4th harmonics through their nonlinear interaction in suitably chosen crystals.

We use two beta-barium borate $\left(\beta-\mathrm{BaB}_{2} \mathrm{O}_{4}, \mathrm{BBO}\right)$ crystals to first double frequency of the infrared to produce green $(\lambda=527 \mathrm{~nm})$ and then double again frequency of the green to produce UV $(\lambda=264 \mathrm{~nm})$. These UV photons are sufficiently energetic to extract electrons from the photocathode with very good quantum efficiency, of about $2 \%$. 


\section{B.2 Theoretical Background}

We approximate the laser beam as a Gaussian in both the longitudinal and transverse domains with rms size $\sigma_{t}$ and $\sigma_{r}$. Hence the laser intensity can be expressed as

$$
I=I_{0} \exp \left(-\frac{r^{2}}{2 \sigma_{r}^{2}}\right) \exp \left(-\frac{t^{2}}{2 \sigma_{t}^{2}}\right) .
$$

Here, $I_{0}$ is the peak intensity, $t$ is time, and $r$ is transverse distance. The peak intensity can be found from the beam size and the measured pulse energy $E$,

$$
I_{0}=\frac{E}{(2 \pi)^{3 / 2} \sigma_{r}^{2} \sigma_{t}}
$$

The energy of the pulse at the doubled frequency can be expressed as [58]

$$
E_{2 \omega}=\frac{2}{\Delta \lambda} \int_{-\infty}^{\infty} d t \int_{0}^{\frac{\Delta \lambda}{2}} d \lambda \int_{0}^{\infty} d r 2 \pi r I \tanh ^{2}\left(\sqrt{I l^{2} K h(\lambda, \theta)}\right),
$$

where $K=K_{0} /\left(1+l / l_{a}\right)$ is the walk-off corrected material constant, and $l_{a}=$ $2 \sigma_{r} \sqrt{\pi} / \rho$ is the effective crystal length over which harmonic generation can take place, referred to as the aperture length [58]. The angular phase matching is limited due to the divergence of the beam (introduced by the wave front curvature $R$ ), tilt of the crystal $\theta$ and wavelength mismatch (each wavelength $\lambda$ in a bandwidth $\Delta \lambda$ requires a particular matching angle). The limitation of angular phase matching is represented by function $h(\lambda, \theta)$ :

$$
h(\lambda, \theta)=\frac{\sin \left(1.39 l\left[\lambda / \sigma_{\lambda}+(\theta+r / R) / \sigma_{l}\right]\right)}{1.39 l\left[\lambda / \sigma_{\lambda}+(\theta+r / R) / \sigma_{l}\right]} .
$$

Refer to Table B.1 for the full list of parameters and their description.

\section{B.3 Numerical simulation}

We have developed a MatLab program, which calculates the triple integral in Eq. B.3 taking into account the angular phase matching function $h(\lambda, \theta)$. The 
Table B.1: Harmonic generation parameters.

\begin{tabular}{|l|l|l|}
\hline$\Delta \lambda$ & $5 \AA$ & laser bandwidth \\
\hline$\sigma_{r}$ & $0.25 \mathrm{~mm}$ & transverse size $(\mathrm{rms})$ \\
\hline$\sigma_{t}$ & $2.115 \mathrm{ps}$ & pulse length $(\mathrm{rms})$ \\
\hline$l$ & $10 \mathrm{~mm}$ & crystal length \\
\hline$K_{0}^{(g)}$ & $230000 W^{-1}$ & BBO material constant (IR to green) \\
\hline$K_{0}^{(u v)}$ & $914000 W^{-1}$ & BBO material constant (green to UV) \\
\hline$\rho$ & $55.7 \mathrm{mrad}$ & BBO walk-off angle \\
\hline$\sigma_{\lambda}$ & $3.3 \AA$-cm & BBO spectral tolerance \\
\hline$\sigma_{l}$ & $0.25 \mathrm{mrad}-\mathrm{cm}$ & BBO angular tolerance \\
\hline
\end{tabular}

output gives the conversion efficiency as a function of the average intensity of the incoming beam.

Even though the theory predicts a very high conversion efficiency (up to 90\%), such efficiency has never been observed in our experiment (and for many reported results). The non-uniformity of the laser wave front is the main reason for reduced doubling efficiency. We approximate this effect by artificially setting the tilt angle $\theta$ to a non-zero value, which corresponds to the misalignment of the crystal relative to the incoming laser beam. The laser beam was focused onto the first $\mathrm{BBO}$ crystal, hence the radius of the wave front curvature $R=\infty$; the second (quadrupling) $\mathrm{BBO}$ crystal is located $16 \mathrm{~cm}$ away, and there the radius of the wave front curvature is $R \sim 10 \mathrm{~m}$. 


\section{B.4 Results}

The simulations and experimental data for the efficiency of frequency doubling (top) and frequency quadrupling (bottom) are shown in Fig. B.1. The nonuniformity of the wave front is modeled by the tilt angle. As we can see, we need a value for the tilt angle to lie between 0.30 and $0.35 \mathrm{mrad}$ for IR to green conversion. We attribute this to the non-uniformity of the IR wave front. For the green to UV conversion we need the value of the tilt angle between 0.33 and 0.38 mrad. The slight increase of the simulated tilt angle is attributed to the wave front degradation after the first conversion. The results have also been shown in Fig. 2.9.

\section{B.5 Conclusion}

We have measured the second harmonic generation efficiency for both green and UV production. The best conversion efficiency for the green is $52 \%$, and for the UV it is $40 \%$. The overall efficiency for 4 th harmonic generation (IR to UV) is $21 \%$. We clearly see the saturation of the UV crystal, which we attribute to high peak intensity. The experimental data matched well with the simulation. The agreement is especially good for the unsaturated case of 2nd harmonic generation (IR to green). 

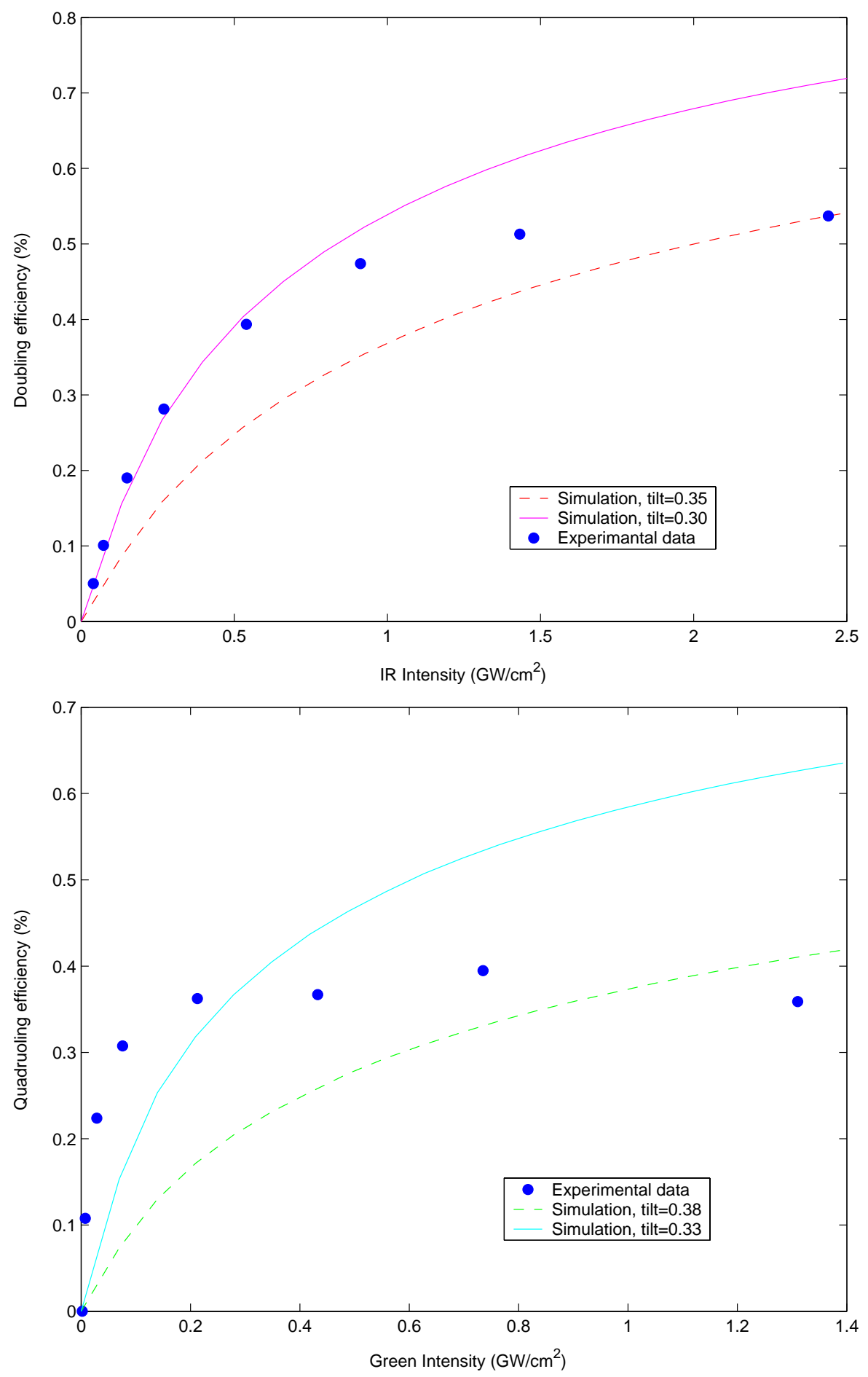

Figure B.1: Doubling (top) and quadrupling (bottom) efficiency simulations and experimental data. The non-uniformity of the wave front is modeled by the tilt angle. 